\title{
Le maïs au Mexique dans les collections du Musée de l'Homme
}

I - Le maiis et sa culture $-1^{\mathrm{e}}$ partie

"El maíz en México" en las colecciones del Museo del Hombre. I - El maíz y su cultura

\section{Pauline Rameau et Serge Bahuchet}

\section{OpenEdition}

\section{Journals}

Édition électronique

URL : https://journals.openedition.org/ethnoecologie/7175

DOI : $10.4000 /$ ethnoecologie. 7175

ISSN : 2267-2419

\section{Éditeur}

Laboratoire Éco-anthropologie

\section{Référence électronique}

Pauline Rameau et Serge Bahuchet, "Le maïs au Mexique dans les collections du Musée de l'Homme », Revue d'ethnoécologie [En ligne], Supplément 2 | 2021, mis en ligne le 25 novembre 2021 consulté le 20 décembre 2021. URL : http://journals.openedition.org/ethnoecologie/7175; DOI : https://doi.org/10.4000/ethnoecologie.7175

Ce document a été généré automatiquement le 20 décembre 2021.

\section{c) $(9)$}

Revue d'ethnoécologie est mis à disposition selon les termes de la licence Creative Commons Attribution - Pas d'Utilisation Commerciale - Pas de Modification 4.0 International. 


\title{
Le maïs au Mexique dans les collections du Musée de l'Homme
}

\author{
I - Le maïs et sa culture $-1^{\mathrm{e}}$ partie \\ "El maíz en México" en las colecciones del Museo del Hombre. I - El maíz y su \\ cultura
}

Pauline Rameau et Serge Bahuchet

«C'est ainsi que la nourriture fut découverte, et cette dernière forma la chair de l'Homme créé, de l'Homme formé. C'est elle qui composa son sang c'est à partir d'elle qu'est né le sang de l'Homme.

C'est ainsi que le maïs est entré ; à travers le travail des Géniteurs, le maïs permit alors la formation de l'Homme.

En broyant neuf foix les épis de maïs jaunes et les épis de maïs blancs, Ixmucané créa une boisson, de laquelle découlèrent la force et la corpulence. Ainsi furent formés les muscles et la vitalité de l'Homme - fruits des Géniteurs que l'on nomme Tepeu et Gucumatz. Par la suite, eux se rassemblèrent afin d'évoquer

la création et la formation de notre première mère et de notre premier père. De maïs jaune et de maïs blanc on façonna leur chair ; avec la pâte

de maïs on fabriqua les bras et les jambes de

l'Homme. La chair de nos parents naquit de la seule farine de maïs. »

Extrait de Popol Vuh. Las antiguas historias del Quiché (éd. Recinos) : 103-104, traduit de l'espagnol et adapté par nous. 


\section{Histoire des collectes et collections mexicaines}

1 Plante aux multiples visages, le maïs est présent partout dans le monde. Dans les collections d'ethnobotanique, il apparait pour la première fois en 1899 avec des épis collectés au Bénin par Auguste Chevalier (cf. Bahuchet et al. 2019 : § 55).

2 Au Mexique, nos collectes ethnoécologiques commencent en 2005 avec le début de la collaboration entre le laboratoire d'éco-anthropologie du Muséum et l'Université de Guadalajara (Autlán) au Mexique (cf. Aubaile-Sallenave 2021); elles s'orientent résolument vers les objets qui accompagnent la vie quotidienne mexicaine. Du point de vue statutaire, ces collections constituent du matériel d'étude, rattaché au laboratoire d'éco-anthropologie. Elles sont inventoriées sous le préfixe ETB- (« EThnoBiologie »).

Les collections patrimoniales d'ethnologie du Muséum, inventoriées sous le préfixe MNHN-E (pour « Ethnologie »), se sont constituées à partir de 2002 après la dévolution au Musée du quai Branly-Jacques Chirac des collections historiques d'ethnologie non européenne du laboratoire d'ethnologie du Musée de l'Homme. Elles comportent également quelques objets originaires du Mexique, dont cependant très peu concernent le maïs ; ils seront eux-aussi inclus dans ce catalogue.

4 Notre matériel d'étude mexicain compte actuellement plus de 850 objets et spécimens ${ }^{1}$, parmi lesquels des plantes cultivées (plus de 170 échantillons de haricots, piments et maïs). Les domaines les plus importants concernent l'alimentation et la cuisine (350 objets), l'agriculture et l'élevage (100 objets), la fête des morts ( 120 objets), l'artisanat et la vannerie (50 objets). Dans tous ces ensembles, les objets touchant au maïs sont nombreux, ce qui nous a engagé à en dresser un inventaire spécifique; ils concernent tant l'agriculture, l'alimentation, la vannerie que les aspects festifs et symboliques.

C'est en 2013 que la collection mexicaine prend un tournant résolument «Zea mays » avec les travaux menés sur la milpa dans le Jalisco; en 2014 celle-ci s'agrandit considérablement, lors de la collecte effectuée en vue de l'élaboration de la nouvelle galerie du Musée de l'Homme (Figure 1). 
Figure 1 : Les variétés de maïs collectées dans différents États du Mexique (5/12/2014, San Cristobal de las Casas, Chiapas)

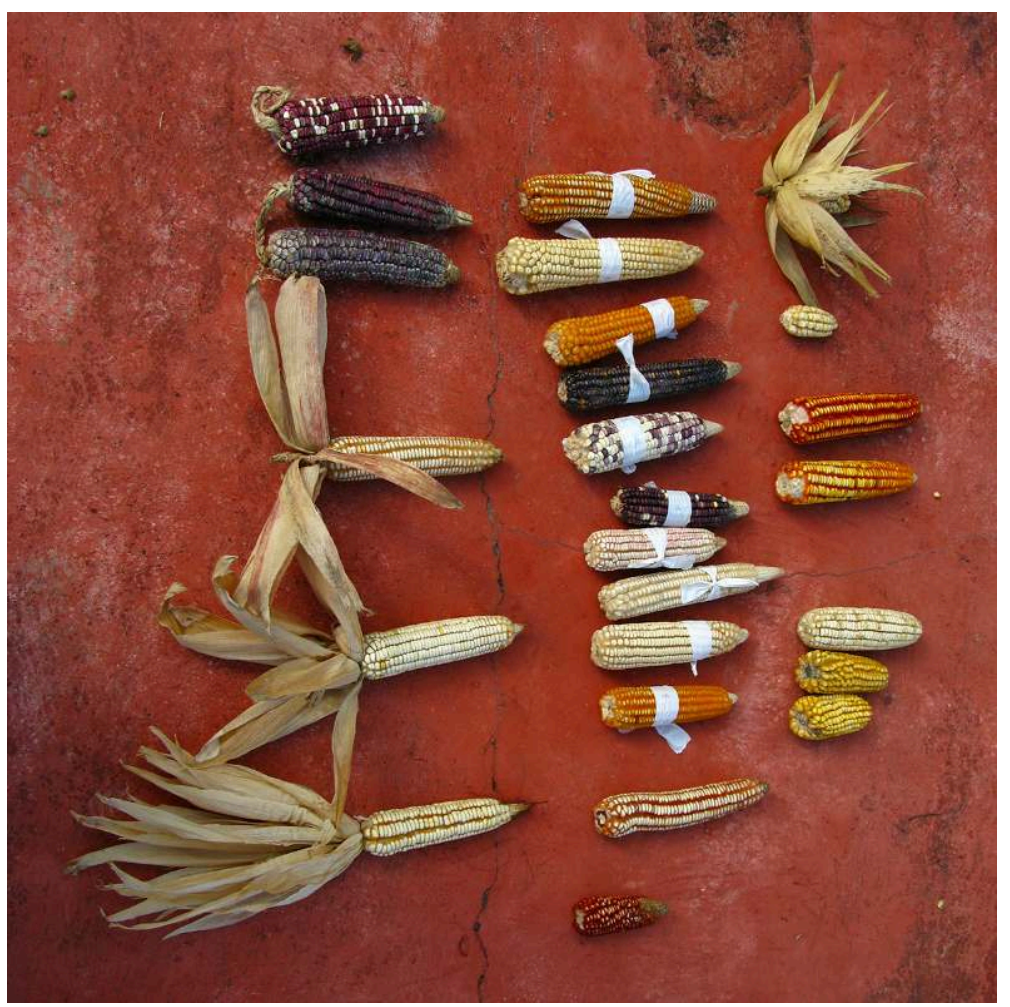

(C) P. Rameau

6 Parmi les étudiants effectuant leur stage de fin de Master $^{2}$ au Mexique, plusieurs réalisent leur recherche de terrain dans des communautés rurales et abordent plus précisément le sujet du maïs (Leal-Almaraz 2008, Rameau 2013, Zurita Benavides 2009 ; Zurita Benavides et al. 2012). Ces recherches concernent la diversité et les usages du maïs, ainsi que sa culture dans la milpa. Elles seront poursuivies et enrichies par des recherches complémentaires ultérieures (Rameau 2013-2018, Leal-Almaraz 2014).

7 Ces travaux menés sur le maïs ont fait l'objet d'une vitrine dans l'exposition permanente du Musée de l'Homme portant sur l'agrobiodiversité et les menaces qui pèsent sur celle-ci (Musée 2016 : 119) L'exemple du maïs avait été choisi pour illustrer l'impact des changements de techniques agricoles sur la diversité biologique ainsi que sur les pratiques alimentaires. Nous avons décrit cette vitrine en 2016 (Bahuchet et al. 2016: § 50-51). Outre les éléments des collections ethnoécologiques, elle contient également deux objets mexicains appartenant à la collection patrimoniale d'ethnologie. La vitrine met en regard des variétés hybrides commerciales, cultivées dans une large partie du monde le plus souvent sous forme de monocultures mécanisées, avec une diversité de maïs natifs issus d'une même municipalité du Jalisco, Chiquilistlán. Le but est d'illustrer le contexte agricole actuel qui, selon les stratégies économiques et sociales de chacun, mêle cultures de rente uniformisées pour l'élevage, l'approvisionnement des villes et l'exportation d'une part, et d'autre part cultures de variétés locales pour l'autoconsommation, de taille, textures et saveurs différentes, cultivées pour répondre aux aléas climatiques et saisonniers comme aux attentes culturelles et sociales (Figure 2). 
Figure 2 : Vitrine sur la diversité biologique en danger - l'exemple du maïs au Mexique. Maïs de Chiquilistlán, Jalisco, Mexique (Collection Renata Leal) (Musée de l'Homme, Paris, 2019)

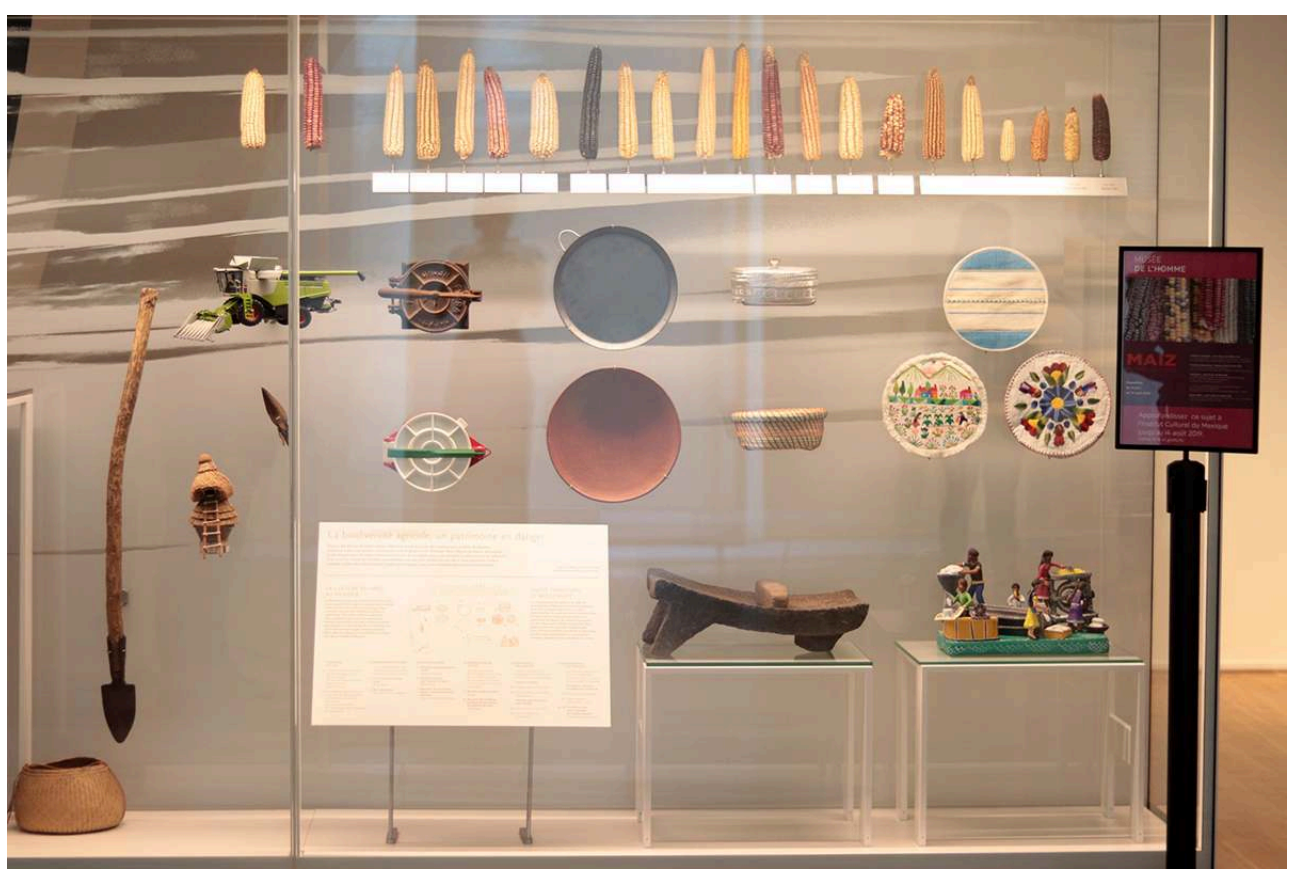

(c) P. Rameau

De ce travail de recherche, mené principalement dans le Jalisco mais aussi dans d'autres régions du Mexique, est née une exposition, Maíz de mis amores, présentée à l'Institut culturel du Mexique à Paris, du 14 juin au 14 août 2019 ainsi que sous une forme réduite du 28 octobre au 2 novembre 2019, dans le cadre d'une manifestation autour de la fête des morts, au sein des locaux de l'UNAM Francia dans le cloître des Cordeliers de la Sorbonne (voir le descriptif de cette exposition dans Bahuchet, Rameau, Arriaga \& Angeles, en préparation).

Enfin, c'est pour l'élaboration du stand de feria mexicaine de l'exposition temporaire Je mange donc je suis ${ }^{3}$ (octobre 2019/juin 2020) qu'une nouvelle collecte a été effectuée entre les États de Tlaxcala et du Michoacán (Figures 3, 4). 
Figure 3 : Fiesta del maïz (13/4/2019, San Juan Ixtenco, Tlaxcala)

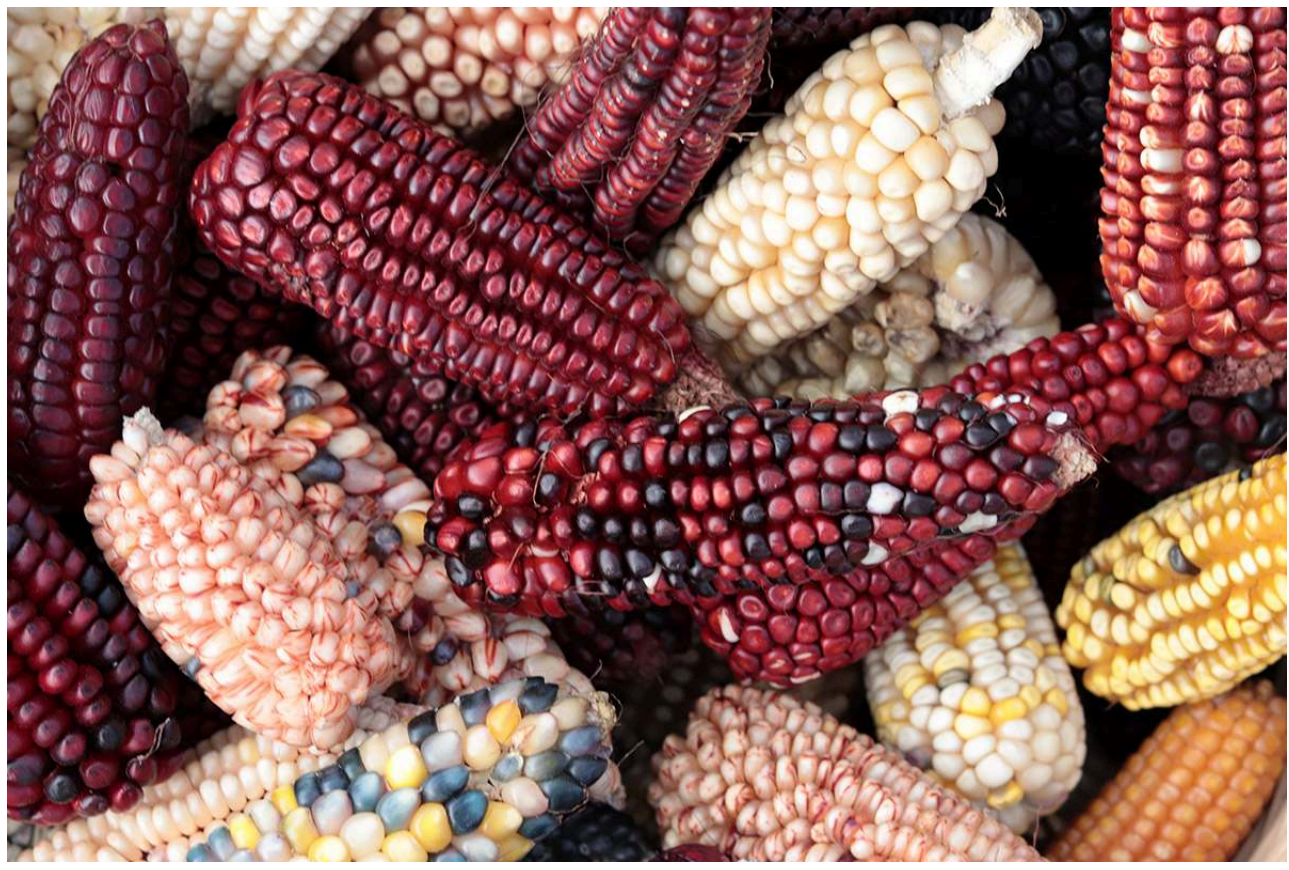

(c) P. Rameau

Figure 4 : Stand de feria mexicaine dans l'exposition « Je mange donc je suis » (Musée de l'Homme, Paris, 2019)

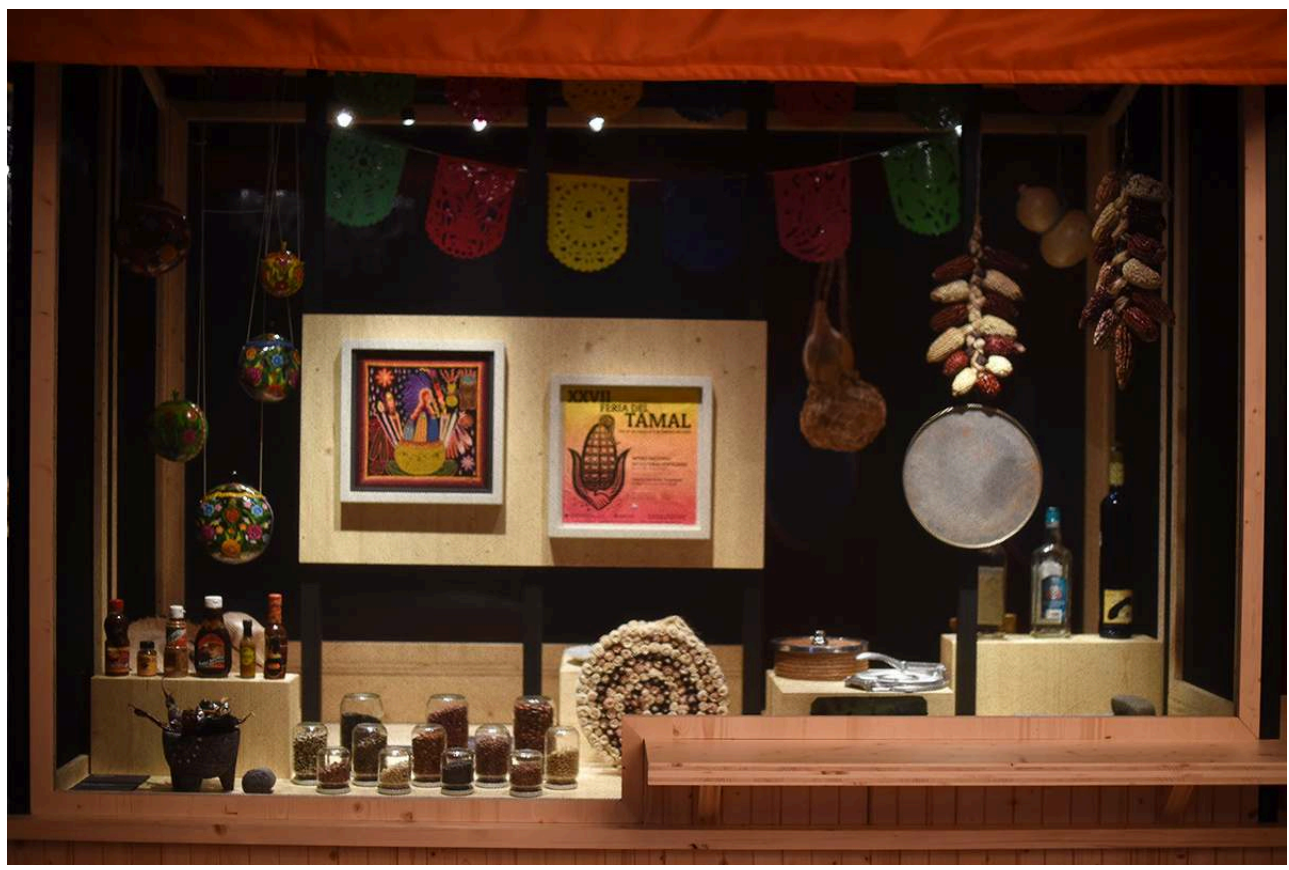

(C) P. Rameau

10 Le présent catalogue rend compte et bénéficie de l'ensemble de ces réalisations muséographiques. 


\section{Méthode et conditions de collecte}

11 Nos enquêtes se sont déroulées auprès des paysans, dans divers villages en grande majorité dans l'État du Jalisco (Figure 5). Nous n'avons jamais cherché à enquêter de manière privilégiée dans des communes autochtones (indigenas); au contraire notre démarche consiste à documenter les usages et la présence du maïs dans la vie quotidienne des Mexicains de toutes origines («mestizos»).

Figure 5 : Entretien sur le terrain dans la coamil de Don Roberto, serpe en main, à Chiquiliztli (17/8/2017, Chiquilistlán, Jalisco)

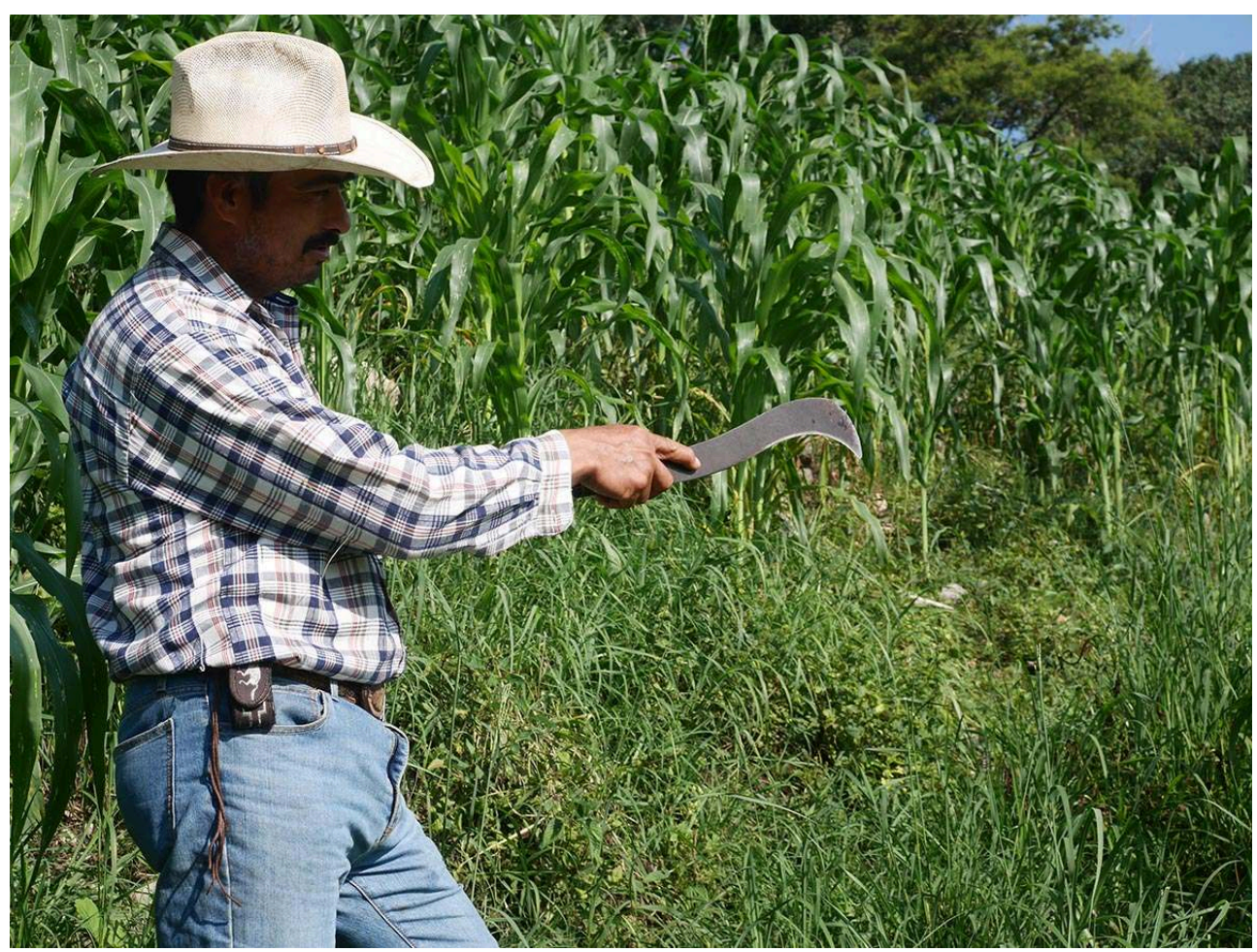

(c) S. Bahuchet

12 En observant les pratiques et les gestes, dans les champs, dans les maisons, dans les cuisines, nous avons évidemment pu prendre connaissance des ustensiles et outils de la vie quotidienne et nous les faire expliquer. Nous avons ainsi visité les lieux où les cultivateurs se fournissent en outillage, tels que les quincailleries, les marchés ou les magasins agricoles (Figures 6, 7, 8). 
Figure 6 : Lieux de collecte : épicerie de village (27/10/2011, Tonaya, Jalisco). Des hottes, des nattes et des balais. Tout au fond, on peut distinguer des gourdes suspendues

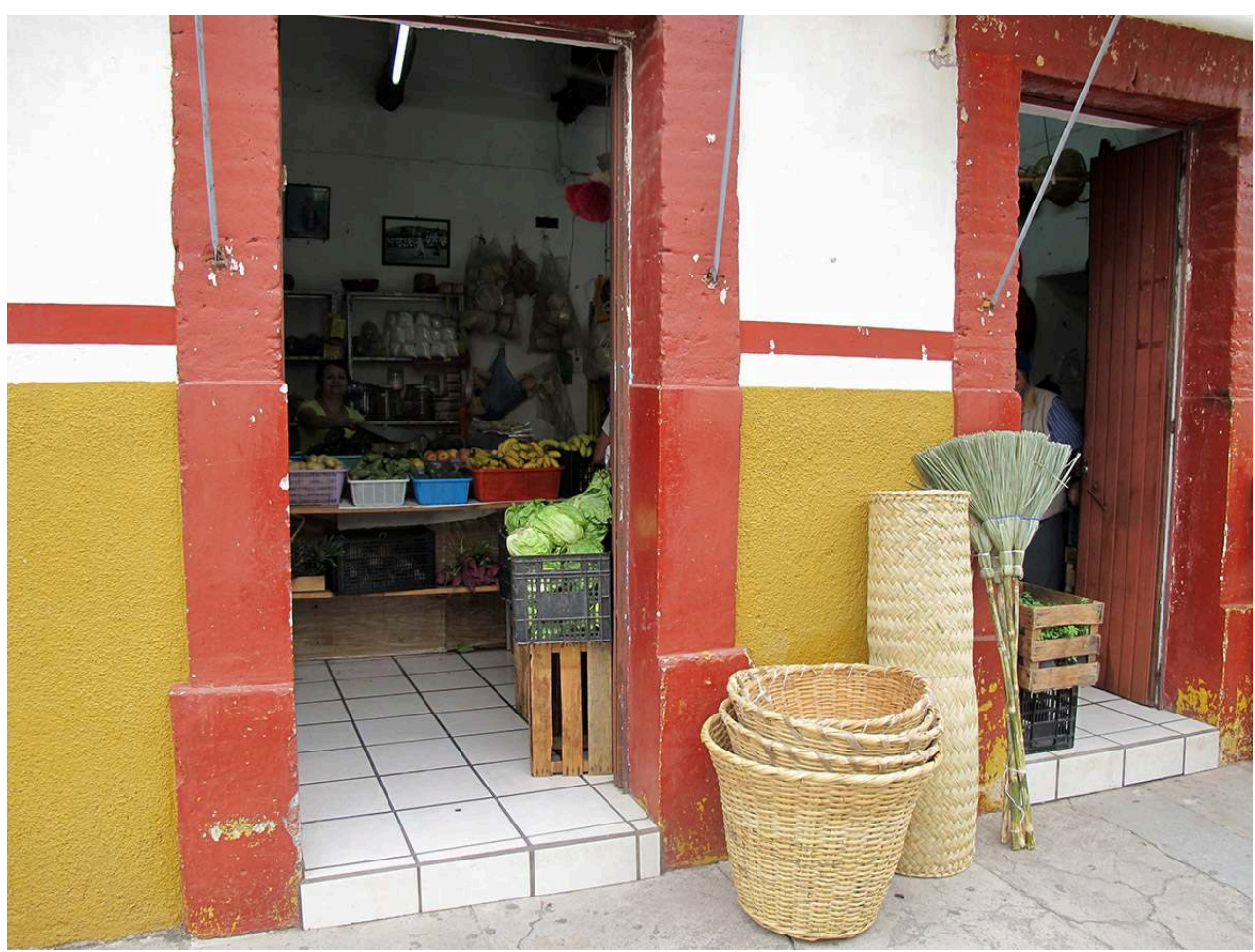

(c) S. Bahuchet

Figure 7 : Lieux de collecte : le marché couvert (21/4/2010, Colima, Colima). Le vendeur de vanneries : les hottes, avec nattes, chaises, corbeilles, éventails...

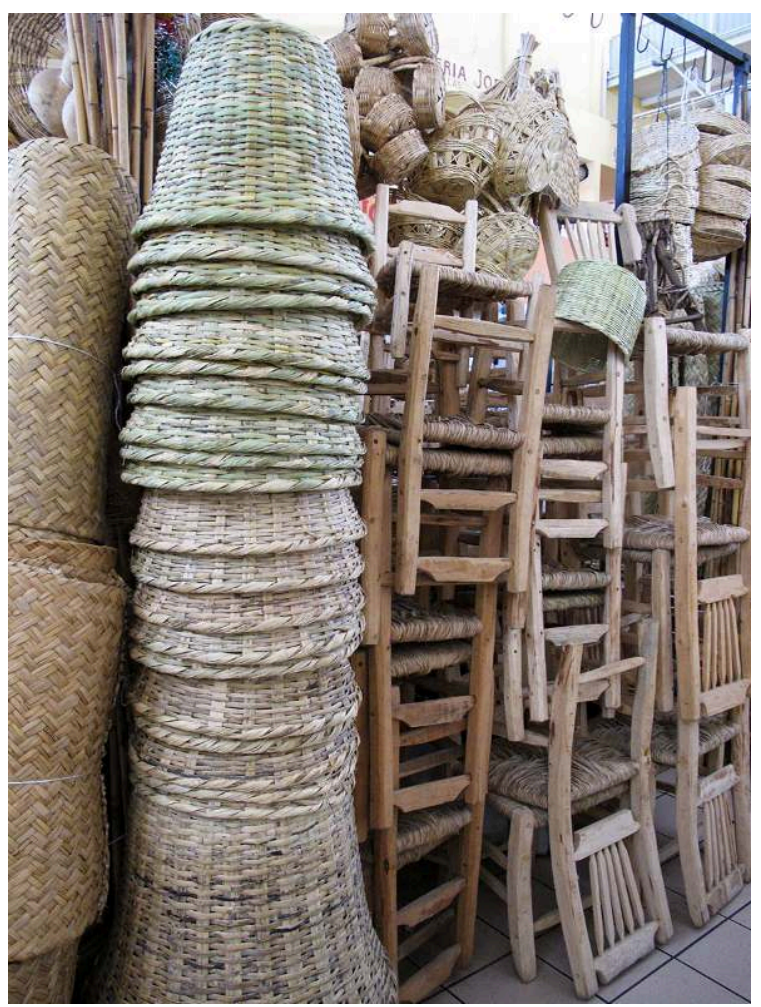

(C) S. Bahuchet 
Figure 8 : Lieux de collecte : le marché couvert (21/4/2010, Colima, Colima). Le quincailler : en haut des fers de bêches, au centre des couteaux-éplucheurs, en bas des lames de serpes

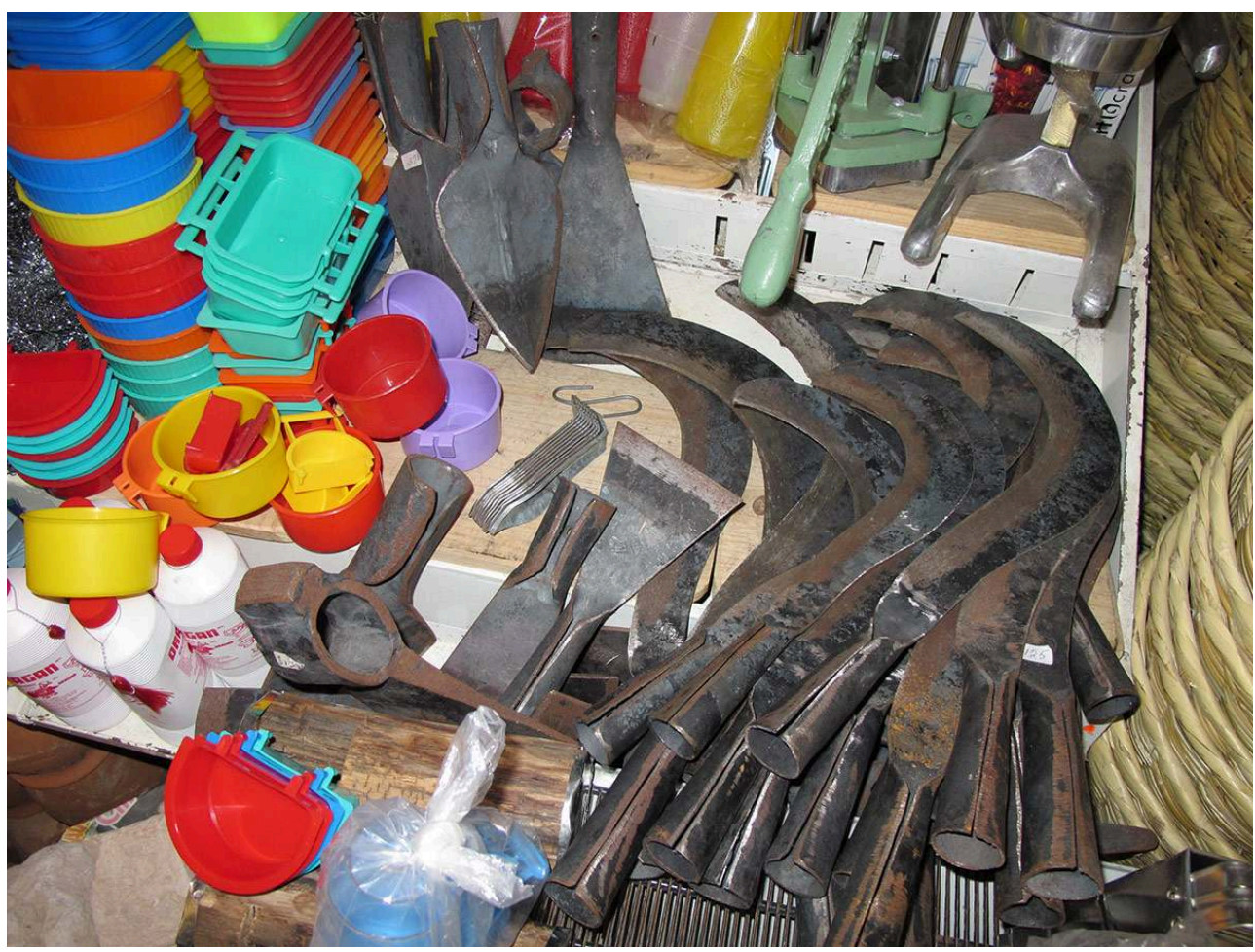

(c) S. Bahuchet

13 Nous avons étendu nos visites et nos collectes aux villes, petites et grandes, auxquelles sont rattachés les villages des cultivateurs, y compris les capitales comme Guadalajara pour l'Etat du Jalisco, ou même Mexico, capitale du Mexique. Notre surprise a été grande d'y trouver des outils fabriqués artisanalement et clairement destinés aux ruraux (Figure 9). 
Figure 9 : Lieux de collecte : quincaillerie (22/3/2012, Guadalajara, Jalisco)

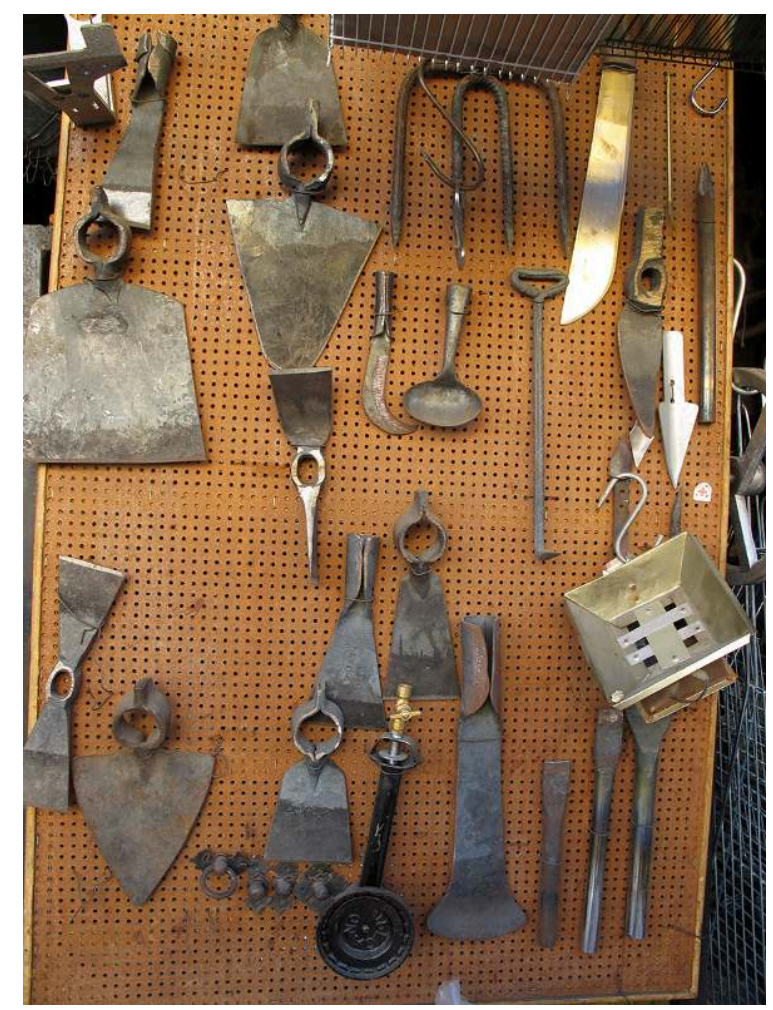

On peut remarquer en haut une machette, à droite un couteau-éplucheur, au centre une lame de serpette non emmanchée (à côté d'un racloir pour les agaves), en bas et à gauche, des fers de houes (c) S. Bahuchet

14 En sus de l'outillage agricole, nous avons acquis dans des boutiques ou des foires spécialisées nombre d'ustensiles issus de l'artisanat, liés à la vie quotidienne, tels que des jouets, des vanneries et tissages en prenant en compte les matières employées, ou encore des figurations telles que des broderies ou des dessins (Figures 10, 13). Beaucoup de ceux-ci concernent la place du maïs dans la vie quotidienne, et nous les présenterons dans la deuxième partie de ce catalogue ("Les usages»). Certains sont destinés à l'usage domestique, alors que d'autres relèvent clairement de la décoration des intérieurs urbains ou sont même destinés aux étrangers. Il n'en demeure pas moins qu'ils véhiculent une certaine image qu'artisans et artistes veulent présenter de leur propre culture. 
Figure 10 : Une boutique de vanneries à Guadalajara (19/11/2010)

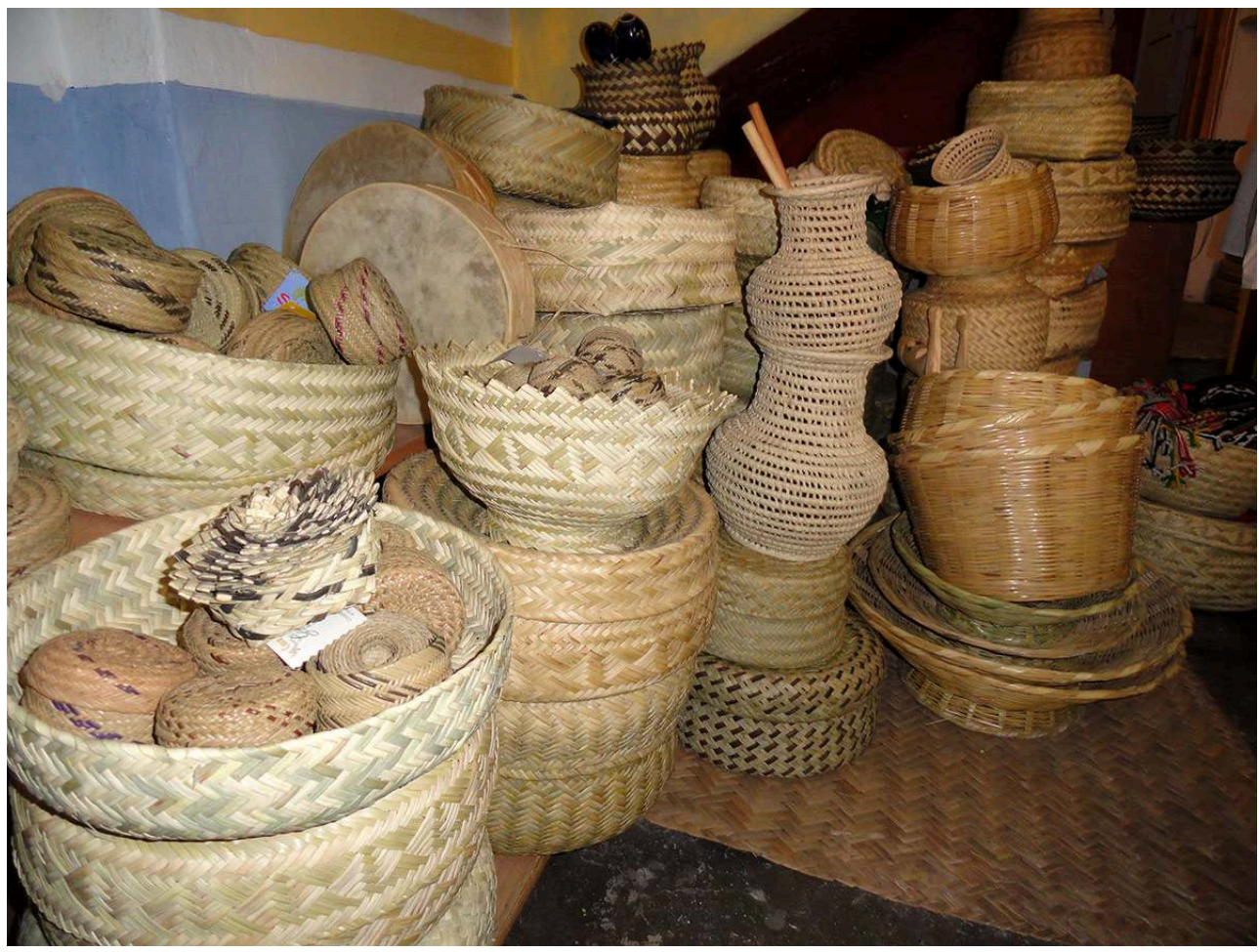

(c) S. Bahuchet

15 L'importance des objets liés au maïs, leur diversité, et le fait qu'ils soient présents dans tous les domaines de la culture matérielle, nous ont convaincus du fait qu'à travers ce catalogue, c'est une certaine présentation de la vie quotidienne du Mexique contemporain que nous esquissons.

\section{Organisation du catalogue}

16 Le catalogue des objets liés au maïs est organisé en deux parties et plusieurs sousparties :

I. La production du maïs

1. La plante et la diversité des variétés

2. L'agriculture

II. Les usages

1. Préparation et consommation

2. Artisanat

3. Représentations.

17 La publication sera échelonnée sur plusieurs livraisons de la revue. La première partie («La production du maïs ») est divisée en deux sections pour des raisons techniques de taille des fichiers; elles paraissent ici l'une à la suite de l'autre.

18 Chaque fiche descriptive suit le même plan :

- Nom de l'objet, suivi du nom local propre au lieu où nous l'avons collecté

- Photographie de l'objet

- Eventuellement communauté ; lieu d'origine (Etat), éventuellement lieu d'acquisition 
si différent

- Description, matière, usage

- Date de collecte ; collecteurs, éventuellement donateurs

- Mesure en $\mathrm{cm}$

- $\mathrm{N}^{\circ}$ d'inventaire

19 L'inventaire est codé de la manière suivante: pour le matériel d'étude, ETB-MX [Mexique]-initiale du collecteur-année-numéro de l'objet dans cette collecte. Pour la collection patrimoniale: MNHN-E-année- $n^{\circ}$ de la collection de l'année- $n^{\circ}$ de l'objet dans cette collection.

20 Aussi souvent que cela nous est apparu pertinent, nous avons accompagné les fiches d'objets par des photographies originales prises sur nos terrains, illustrant le contexte et la manipulation des ustensiles.

\section{La plante et la diversité des variétés}

\section{L'origine du maïs}

21 Le téosinte (Zea mays subsp. parviglumis Iltis \& Doebley, Poaceae; cf. Iltis \& Doebley 1980) est la graminée sauvage considérée comme l'ancêtre du maïs cultivé (Zea mays L., subsp. mays). Il n'est pas rare que les paysans en plantent quelques pieds au bord de leur champ, pour les qualités que le téosinte est censé apporter aux épis de maïs. Originaire de Mésoamérique, le maïs tel que nous le connaissons aujourd'hui a été façonné par la main de l'homme. Depuis sa domestication il y a environ 8000 ans, il occupe une place centrale dans l'agriculture et l'alimentation mexicaine (cf. Staller et al. 2010) (Figure 11). 
Figure 11 : La grotte du premier maïs archéologique (19/7/2017, Cueva del maíz, Coxcatlán, vallée de Tehuacan, Puebla)

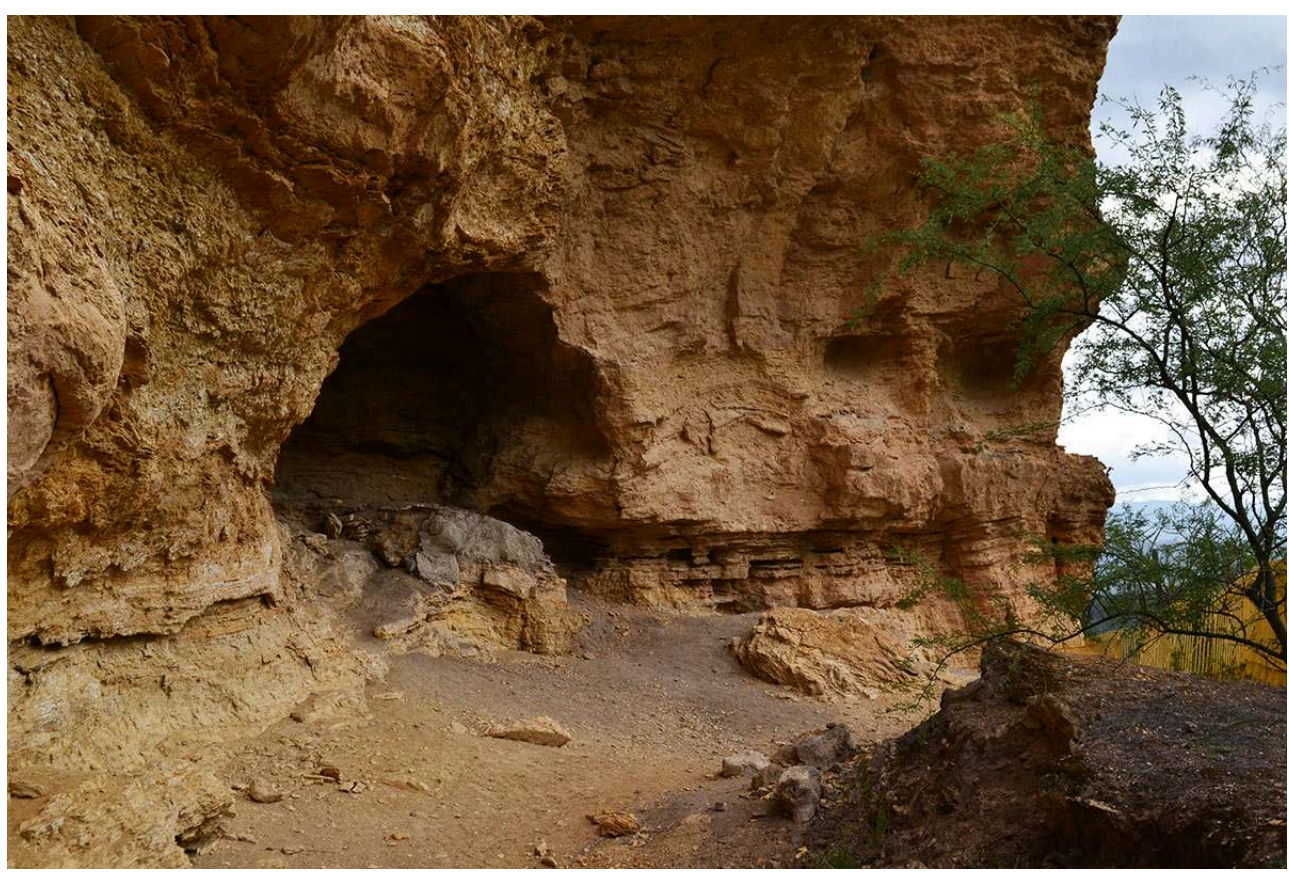

C'est dans cette grotte, et d'autres de cette célèbre vallée de Tehuacan, qu'ont été découverts les plus anciens épis de maïs domestiqué, par l'archéologue Richard MacNeish (1918-2001) et son équipe, dans les années 1960. La séquence chronologique couvre près de 2000 ans, et a révélé une succession de rafles depuis les plus petits, vers 7000 avant le présent, jusqu'à des plus évolués et volumineux, vers 5000 avant le présent (cf. Kato et al. 2009, Staller et al. 2010)

(c) P. Rameau 
Figure 12 : Un épi de téosinte, à côté de deux épis de maïs, dans la maison de Don Lauro $(28 / 11 / 2010$, Chiquilistlán, Jalisco)

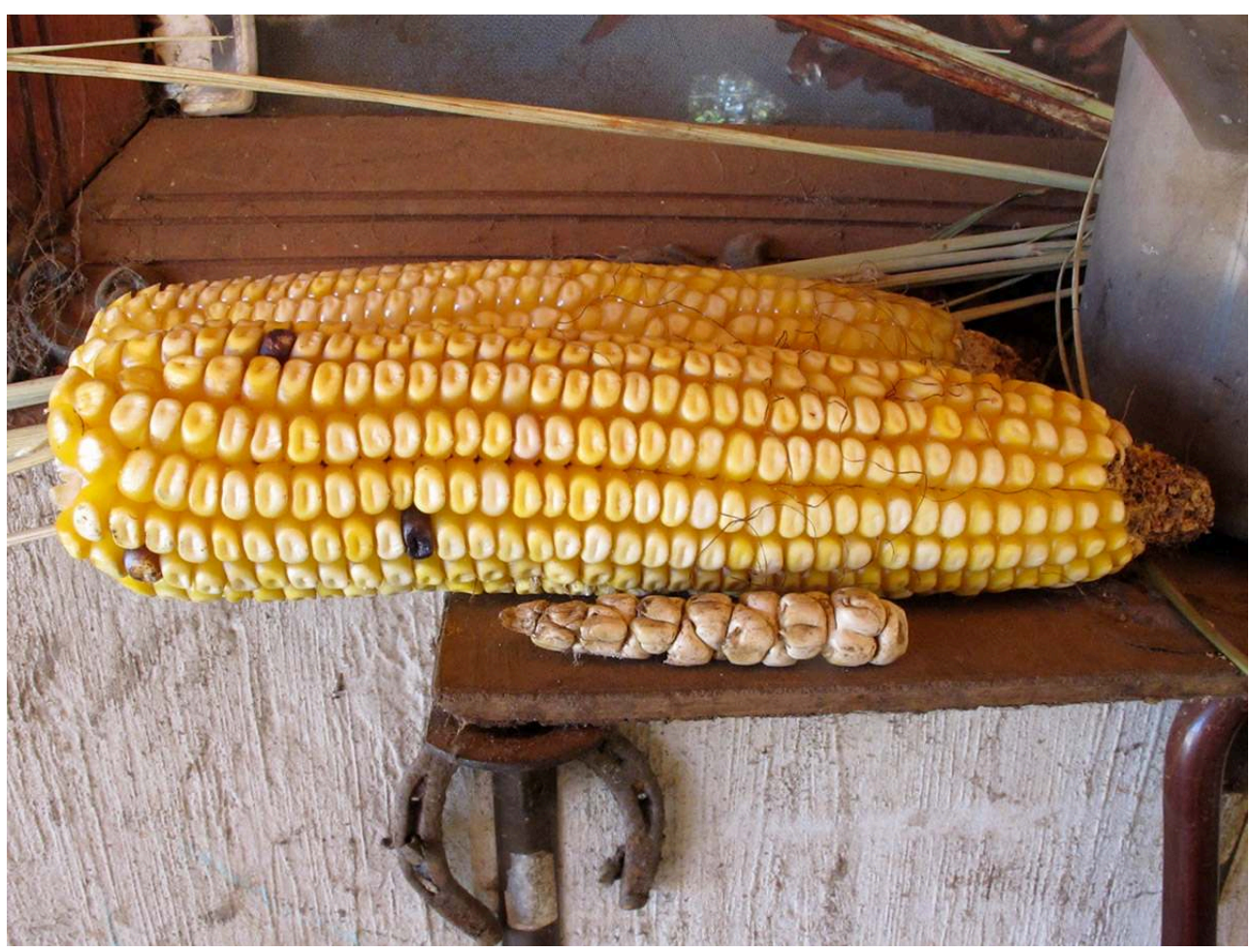

(c) S. Bahuchet

1) Teosintle, broderie 
Figure 13 : Broderie ETB-MX-JLL-2012-39

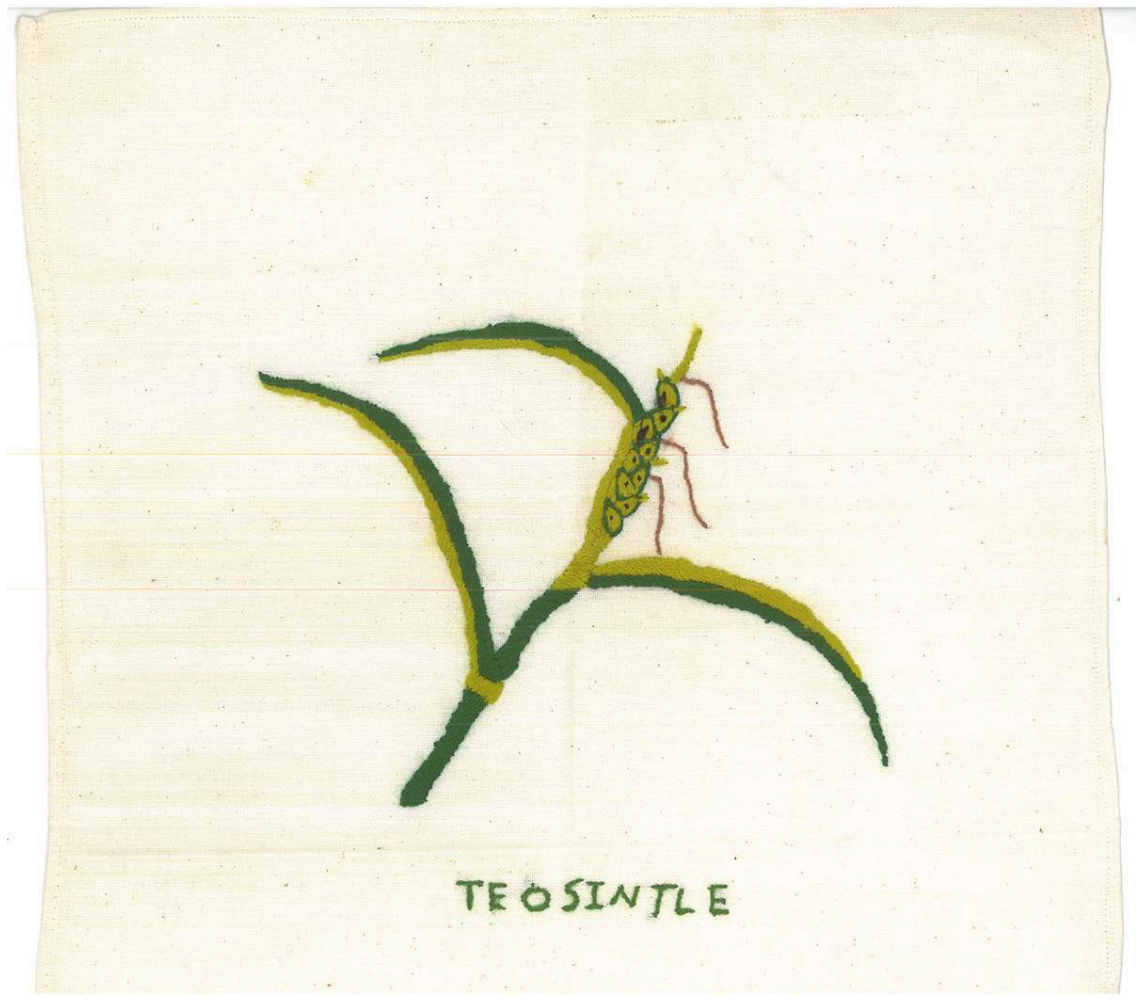

(c) S. Bahuchet

- Cuzalapa (Jalisco)

- Coton brodé

- 2012 ; collecteurs Jean-Marie Betsch et Johana Larco-Laurent

- $31 \times 31 \mathrm{~cm}$

• ETB-MX-JLL-2012-039

\section{La diversité des variétés}


Figure 14 : « Diversidad en el campo » (6/2018, Chiquilistlán, Jalisco)

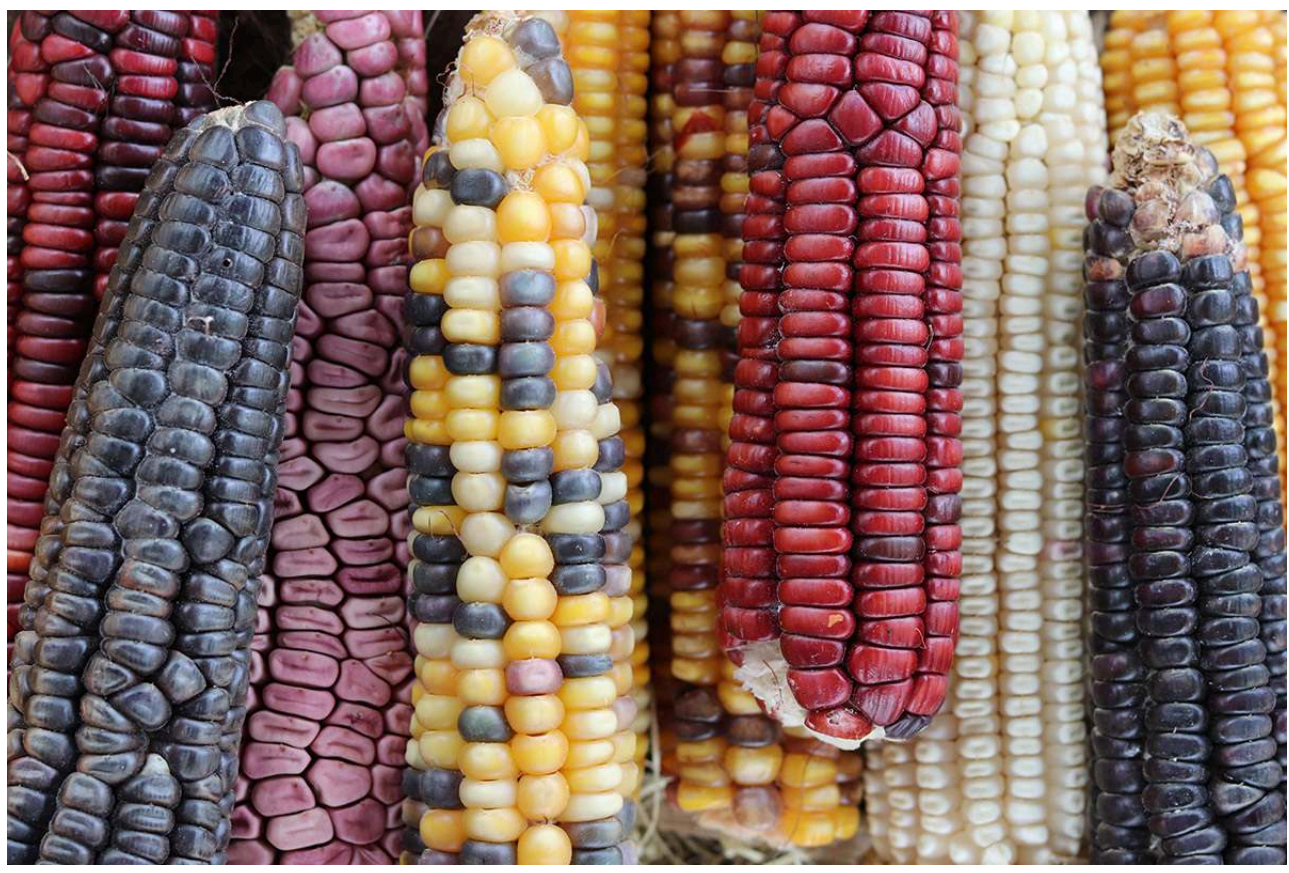

(C) P. Rameau

Plusieurs centaines de variétés locales poussent au Mexique, pays aux climats, altitudes et écosystèmes contrastés (voir les articles dans ce Supplément 2 de la Revue d'ethnoécologie, 2021, en particulier Ortega Paczka, Mota, Perales et Lazos \& Chauvet) (Figure 15). Sur leurs caractères morphologiques et génétiques, on regroupe actuellement les maïs en une soixantaine de "races" (razas), elles-mêmes formant quatre groupes majeurs (CONABIO 2011). Les variétés locales de maïs diffèrent par la taille et le diamètre des épis, la couleur et la forme des grains, le nombre et la linéarité des rangs, mais aussi par la hauteur des tiges (voir par exemple Hernandez X 1985, Kato et al. 2009, Perales \& Golicher 2014, Ron Parra et al. 2006, Sanchez et al. 2000).

2) Affiche de la diversité des maïs « Maices mexicanos » 
Figure 15 : Affiche « Maices mexicanos » ETB-MX-SBPR-2017-086

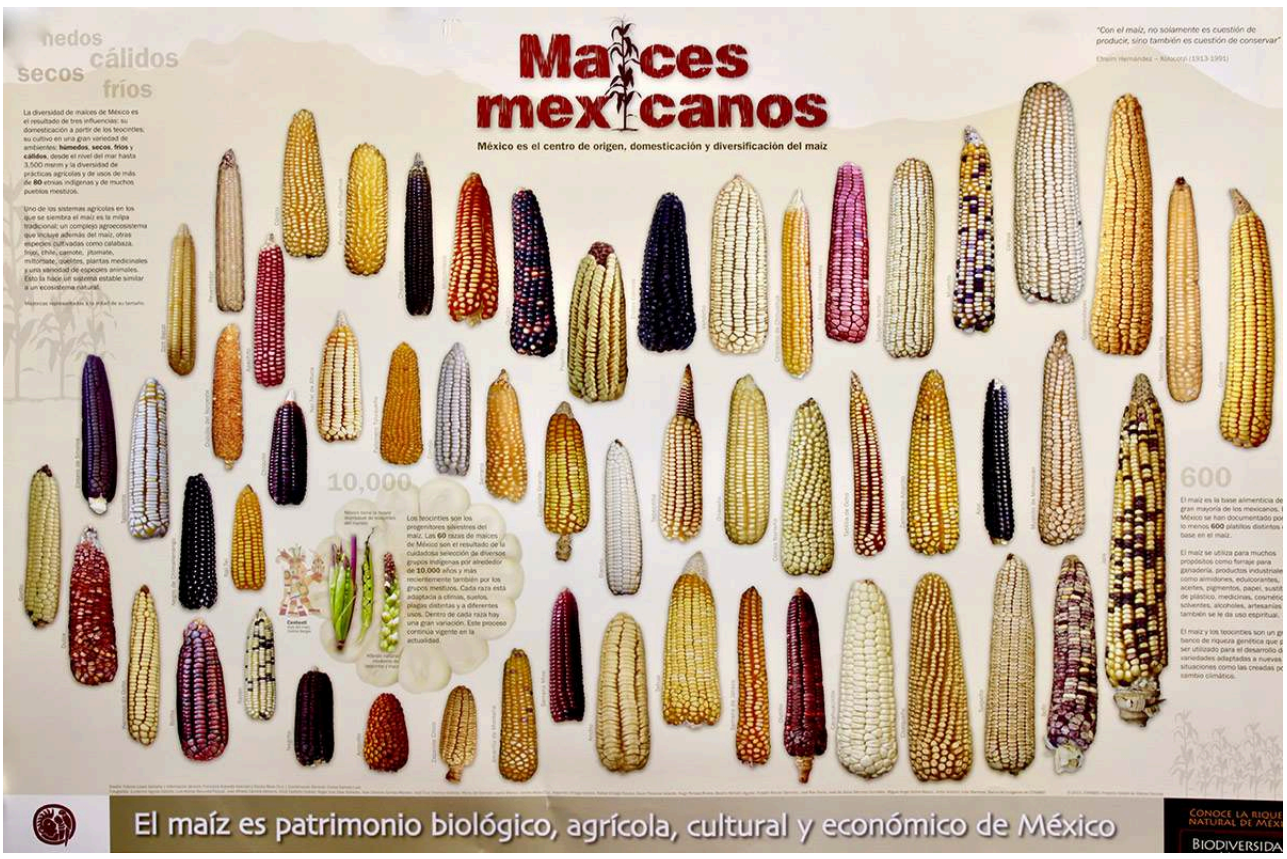

(c) CONABIO, 2013, Proyecto global de maíces nativos

- Mexico (CDMX)

- Impression quadrichromie, « Maíces mexicanos ", produite par la Commission nationale pour la connaissance et l'usage de la biodiversité CONABIO ; « le maïs est le patrimoine biologique, agricole, culturel et économique du Mexique »

- Août 2017 ; collecteurs Serge Bahuchet \& Pauline Rameau

. $60 \times 90 \mathrm{~cm}$

- ETB-MX-SBPR-2017-86

Chaque communauté paysanne plante son propre ensemble de types de maïs, auxquels elle donne ses propres noms, sans que cela coïncide avec la classification en races définies par les spécialistes. La culture de plusieurs variétés de maïs dans des parcelles voisines permet des croisements produisant des épis bigarrés. De tels croisements peuvent également être générés de façon volontaire par les agriculteurs en semant des mélanges des variétés. (Figures 16, 17, 18). La manière de gérer, par les paysans mexicains, les populations hétérogènes et la pollinisation ouverte se distingue clairement de la culture du maïs commercial hybride dont les semences obtenues des lignées pures sont achetées tous les ans. 
Figure 16 : Maïs en fleurs : en haut, les fleurs mâles, en bas les fleurs femelles qui vont donner les épis (16/4/2010, Cuzalapa, Jalisco)

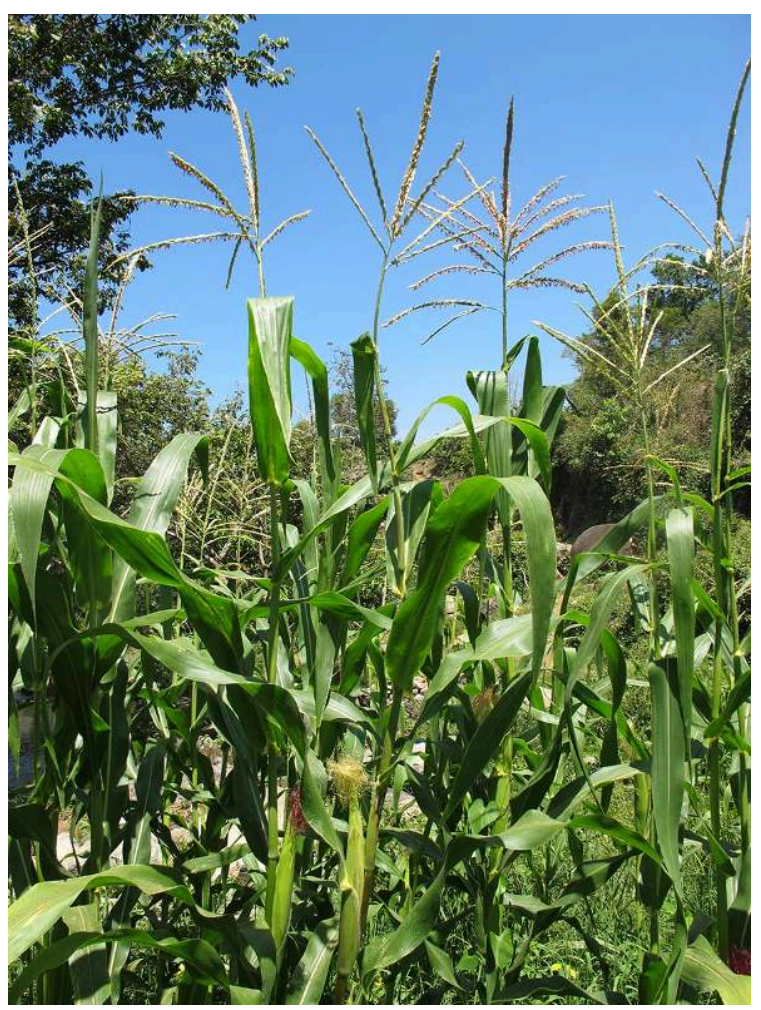

(c) S. Bahuchet

Figure 17 : «Espigas en la milpa », pollinisation croisée (11/9//2017, Chiquilistlàn, Jalisco)

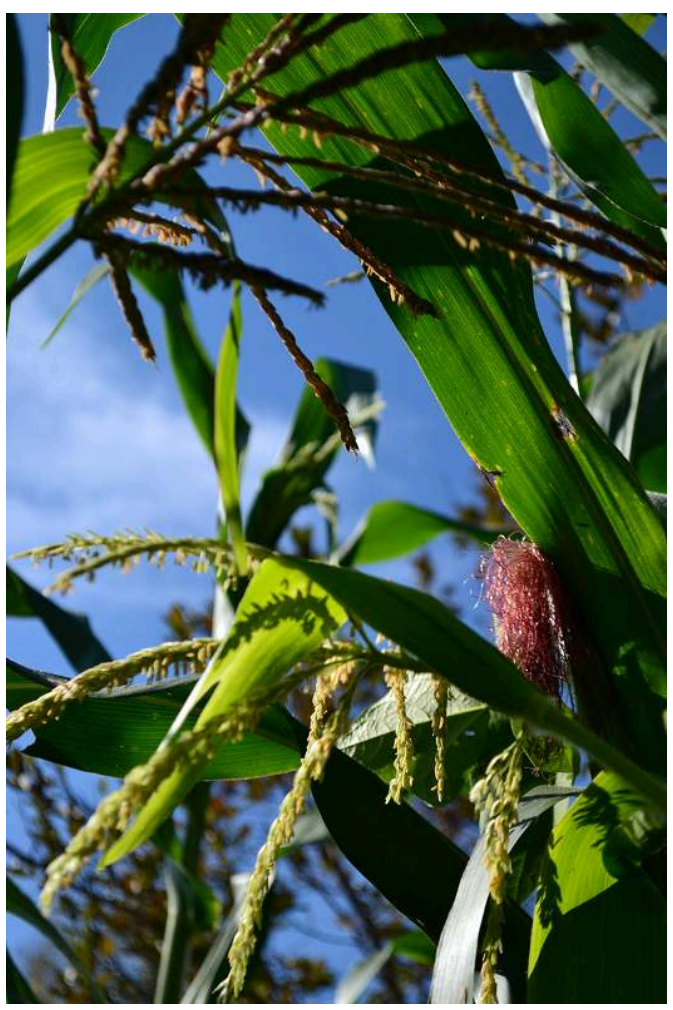

(c) P. Rameau

Revue d'ethnoécologie, Supplément 2 | 2021 
Figure 18 : Les variétés en mélange, gardées pour leurs semences (9/6/2017, Limoncito, Jalisco)

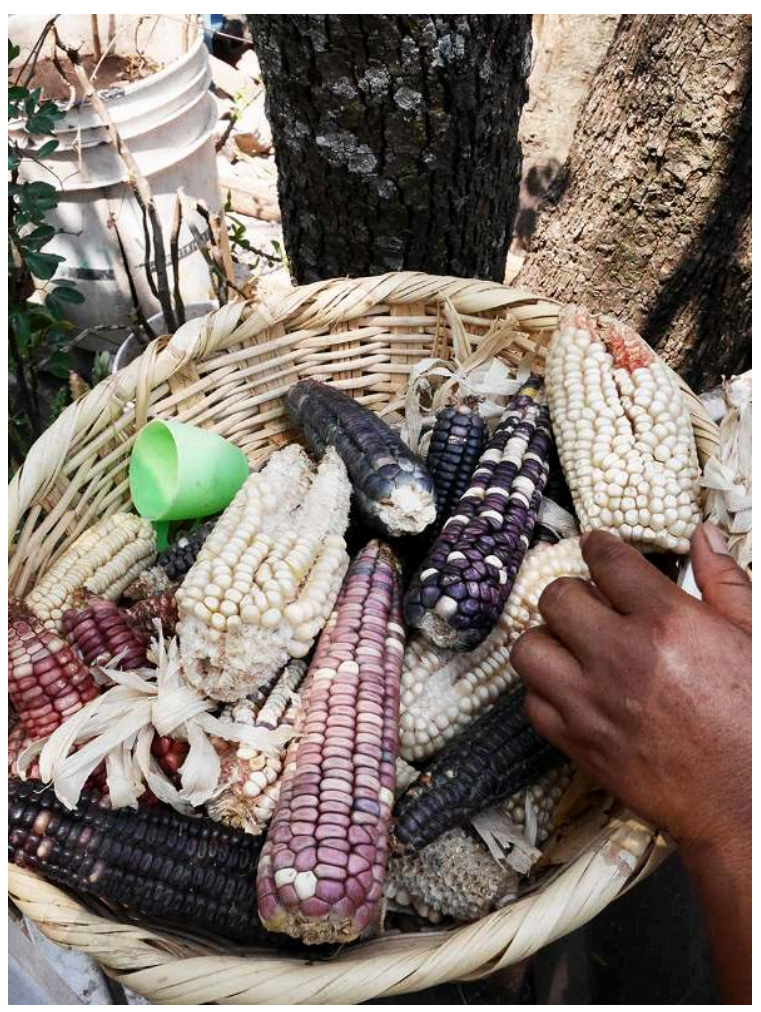

(c) S. Bahuchet

Le développement de l'agriculture intensive et commerciale, et la recherche agronomique sur l'amélioration des plantes introduisent la culture de variétés hybrides commerciales qui entrent en concurrence avec les types locaux, appelés «criollos » ou «nativos » (Figure 19) (voir encadré). 
Figure 19 : “Diversidad del maíz ajo” (13/4/2019, Fiesta del maíz, Ixtenco, Tlaxcala)

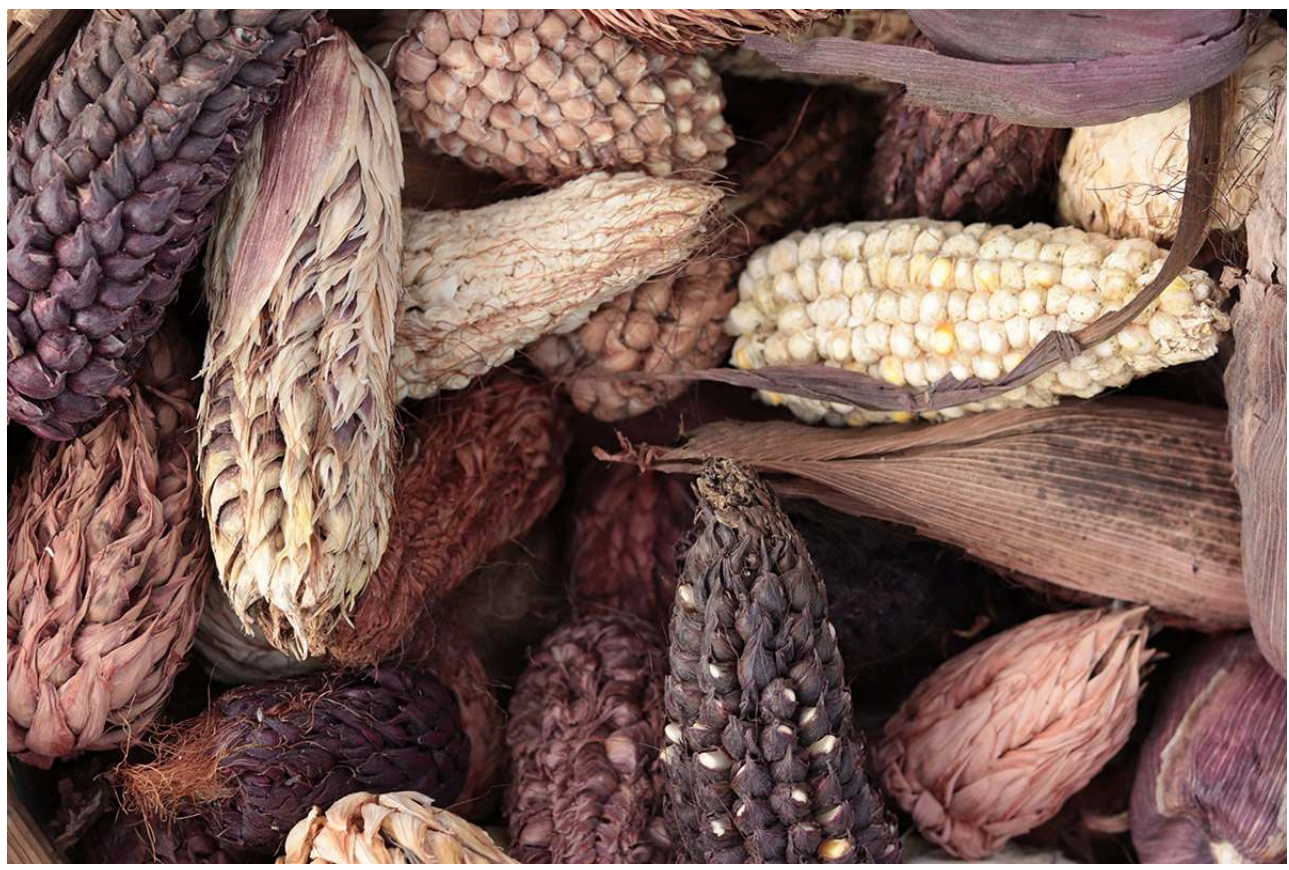

La majorité des épis de cette photo sont des " maïs vêtus », au Mexique ajo tunicado, en anglais podcorn, de la variété tunicata. Ils sont caractérisés par le fait que chaque grain est enveloppé séparément dans une glume.

(c) P. Rameau

Glossaire

Maïs natif : variété originaire de la terre où elle a évolué et développé les caractères qui la différencient des autres variétés.

Maïs créole : variété résultant d'un croisement entre une variété native et une autre étrangère qui s'est acclimatée au terrain local. Localement, les paysans utilisent le terme de " maïs créole " pour désigner en réalité des variétés natives. Maïs hybride ou amélioré : semence issue du croisement de deux lignées pures homozygotes sélectionnées pour leurs caractères avantageux afin d'obtenir une seule et même plante (F1) porteuse des caractères recherchés. Si l'on replante les grains issus de cet hybride, les caractères se dispersent et l'on perd la vigueur hybride - aussi appelée hétérosis - typique de la F1. En raison de ce problème, les semences des hybrides sont donc achetées tous les ans.

Maïs OGM ou transgénique : maïs dont le génome a été modifié pour exprimer des caractères avantageux, tels que la résistance aux herbicides ou aux insectes.

Les épis que nous avons recueillis ne forment aucunement une collection exhaustive, ou représentative des 60 « races » définies par les agronomes; collectés sur nos terrains d'enquête, dans des foires agricoles ou même sur les marchés (y compris à Mexico), ces épis n'ont d'autre but que d'illustrer l'incroyable diversité de ce patrimoine culturel, toujours vivant. Nous ne détaillerons pas ici les quelque 70 variétés de maïs que nous avons collectées, elles devront faire l'objet d'une étude ultérieure (Figure 20, tableau en annexe). 
Figure 20 - Diversité des épis collectés (provenant du Yucatan, du Jalisco et du Tlaxcala), du plus petit $(7 \mathrm{~cm})$ au plus grand $(27 \mathrm{~cm})$

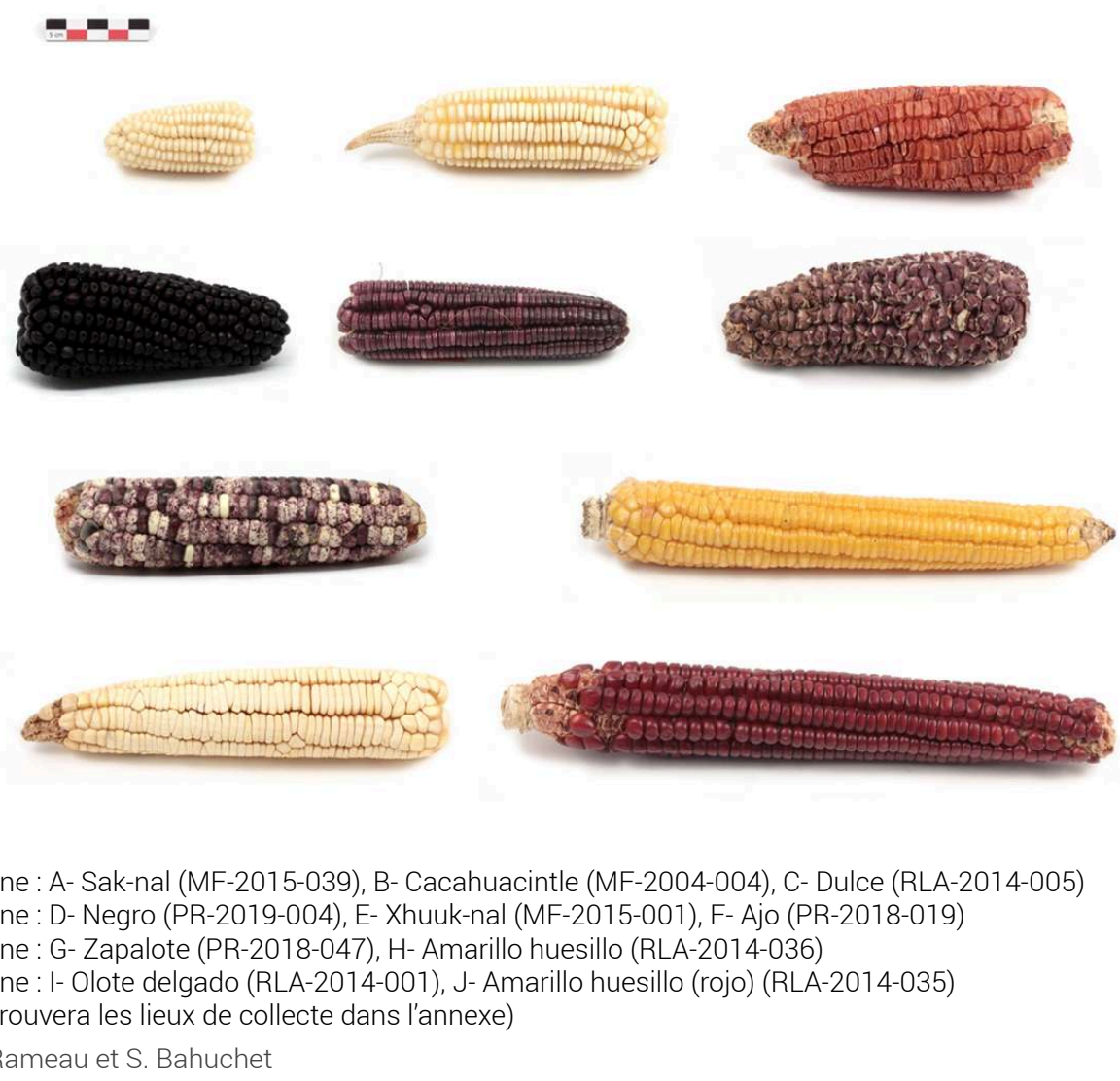

Un gros travail ethnobotanique a été accompli par R. Leal-Almaraz (2008), qui a consisté à inventorier toutes les variétés locales cultivées dans un même village du Jalisco, Chiquilistán. Pour cela elle a enquêté et interrogé la majorité des agriculteurs, en particulier sur leurs motivations pour planter ces cultivars. Elle a ensuite prolongé son inventaire en 2014, pour le compléter, et elle l'a illustré par une affiche destinée en premier lieu aux habitants du village, afin de les sensibiliser à la diversité et donc à la richesse patrimoniale de leur terroir (Figures 21, 22).

3) Affiche des variétés « créoles » de Chiquilistlán 
Figure 21 : Affiche ETB-MX-SBPR-2017-87

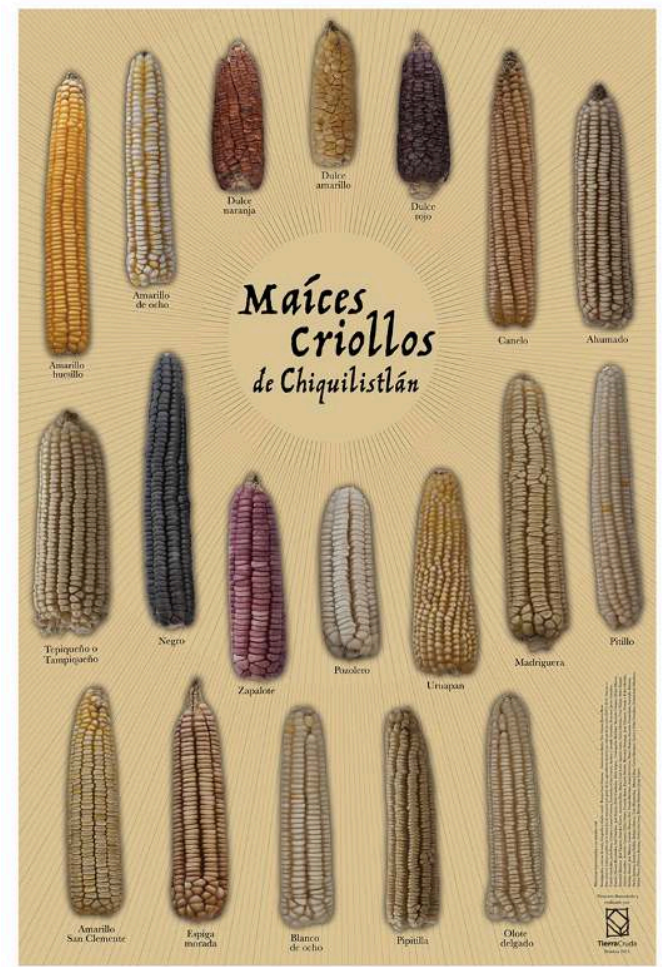

(c) R. Leal-Almaraz

32 - Chiquilistlán/Guadalajara (Jalisco)

- "Maíces criollos de Chiquilistlán ", affiche créée par Renata Leal-Almaraz à la suite des inventaires réalisés par elle de 2008 à 2014, destinée à être diffusée dans le village pour attirer l'attention sur la richesse que représente la diversité des variétés cultivées au village

- Juin 2017 ; collecteurs Serge Bahuchet \& Pauline Rameau, don de R. Leal-Almaraz

- Tirée en deux formats, $30 \times 42 \mathrm{~cm}$ et $60 \times 88 \mathrm{~cm}$

- ETB-MX-SBPR-2017-087 
Figure 22 : Examen de l'affiche par Concepción Martinez, l'un des agriculteurs interrogés au cours de l'inventaire (Limoncito, Chiquilistlán, 8/6/2017)

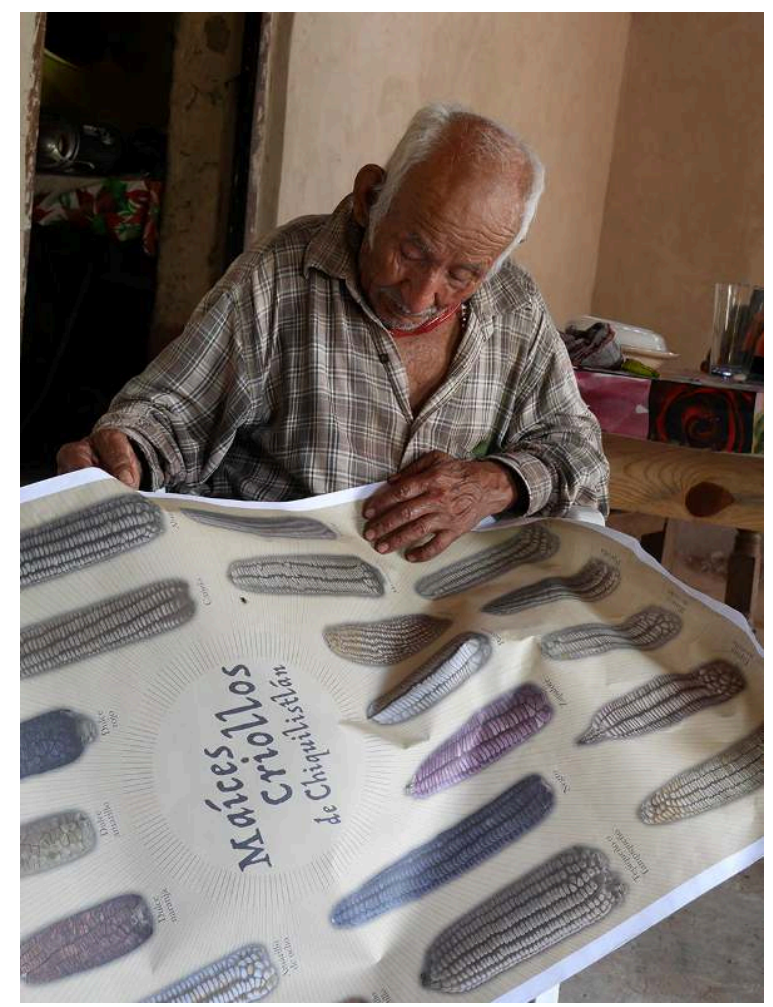

(c) S. Bahuchet \& P. Rameau

D'autres études similaires ont été réalisées par la suite. Un travail mené en 2009 dans deux communautés indigènes du Veracruz par Gabriela Zurita, a été centré sur les choix économiques des paysans (Zurita Benavides 2009, Zurita Benavides et al. 2012). Une étude longitudinale comparative entre 1999 et 2011, a porté sur la dynamique de conservation des variétés dans un village du Yucatán (Fenzi et al. 2017). Une deuxième étude, toujours dans ce même village, a analysé le flux de semences, dynamique qui a permis de rétablir la diversité de maïs cultivés après un événement climatique extrême (Fenzi et al. 2021)

Bien que la culture du maïs transgénique (OGM) soit actuellement interdite au Mexique, les grains qui sont commercialisés pour l'alimentation, importés des USA, peuvent être replantés par les paysans ou accidentellement mélangés aux semences locales, ce qui a provoqué une contamination du maïs natif, détectée dans plusieurs régions du pays (cf. Quist \& Chapela 2001, Dyer et al. 2009). En réaction, de plus en plus de mouvements paysans et citoyens se développent, pour favoriser la culture et la conservation vivante des variétés locales, nativas, des maïs mexicains et s'opposer fermement à la culture des maïs OGM (Figure 23). 
Figure $23: 7^{e}$ rencontre RASA à Ixtlahuacán de los Membrillo, Jalisco $(21 / 11 / 2010)$ : sous la tente de la réunion, un calicot affirme "Les OGM ne sont pas la solution»

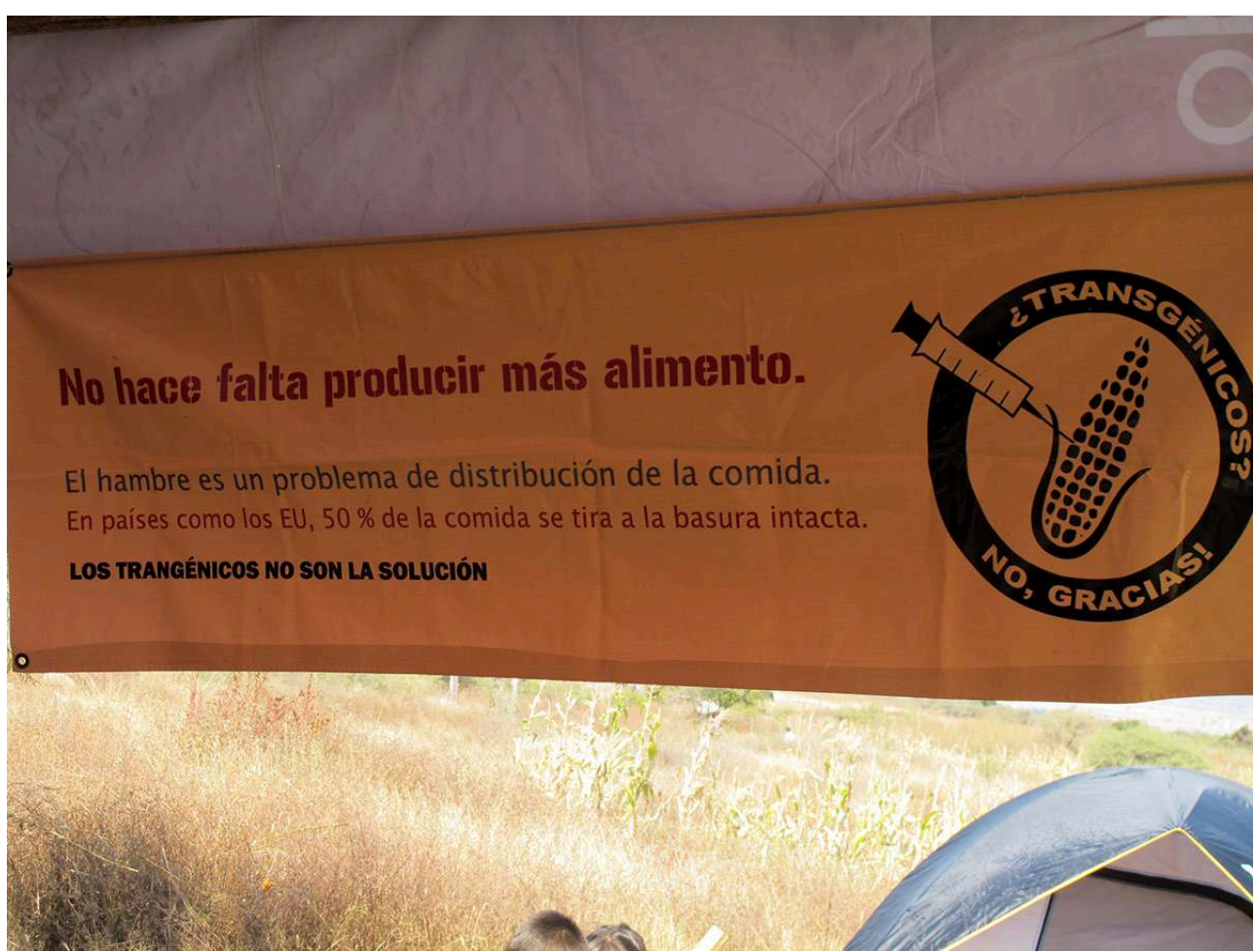

(c) S. Bahuchet

L'un de ces groupes, particulièrement actif au Jalisco, est le RASA, Red de Alternativas Sustentables Agropecuarias " réseau des alternatives agropastorales durables ». Il réunit annuellement des paysans et des consommateurs autour du thème "Nuestro maiz; nuestra cultura ", qui discutent des problèmes, dangers et réalisations, échangent leurs expériences, montrent leurs productions et la diversité des plantes cultivées (maïs, haricots, tomates et autres légumes...), en même temps que l'examen des maïs ayant été plantés antérieurement permet d'en évaluer les caractères et d'en prélever des semences pour ceux qui le souhaitent (Figures 24, 25). 
Figure $24: 7^{\mathrm{e}}$ rencontre RASA à Ixtlahuacán de los Membrillo, Jalisco (21/11/2010) : discussions, échange d'expérience, et de semences

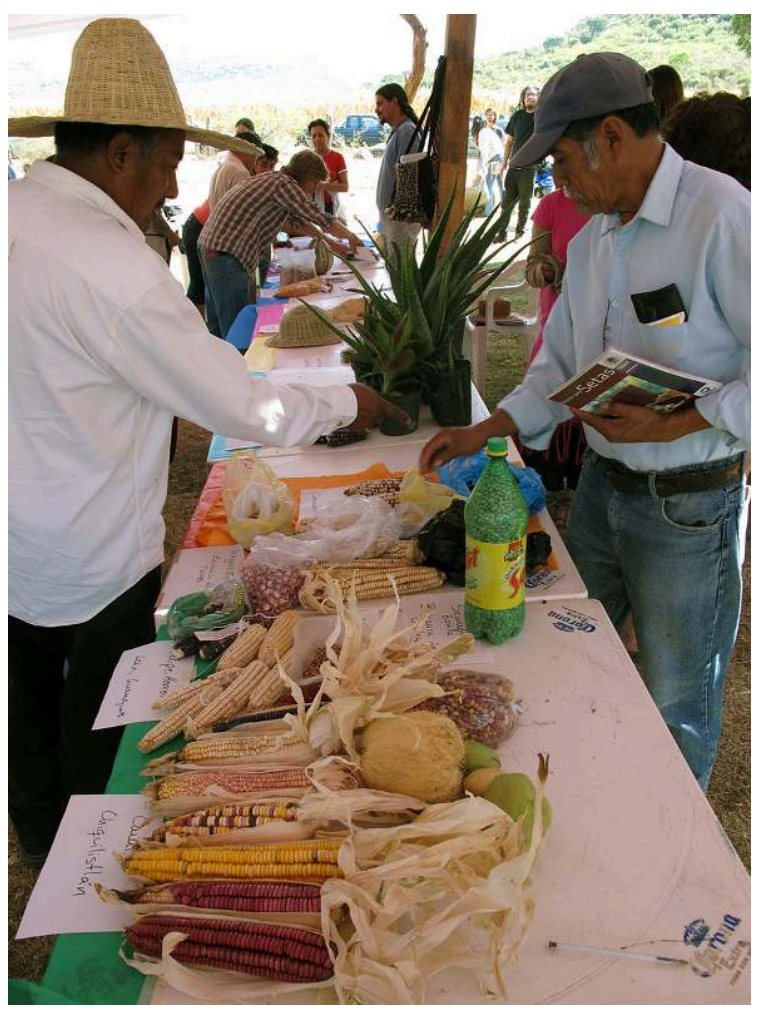

(c) S. Bahuchet

Figure $25: 7^{\mathrm{e}}$ rencontre RASA à Ixtlahuacán de los Membrillo, Jalisco $(21 / 11 / 2010)$ : visite des parcelles cultivées, discussion sur les diverses variétés et dons de graines

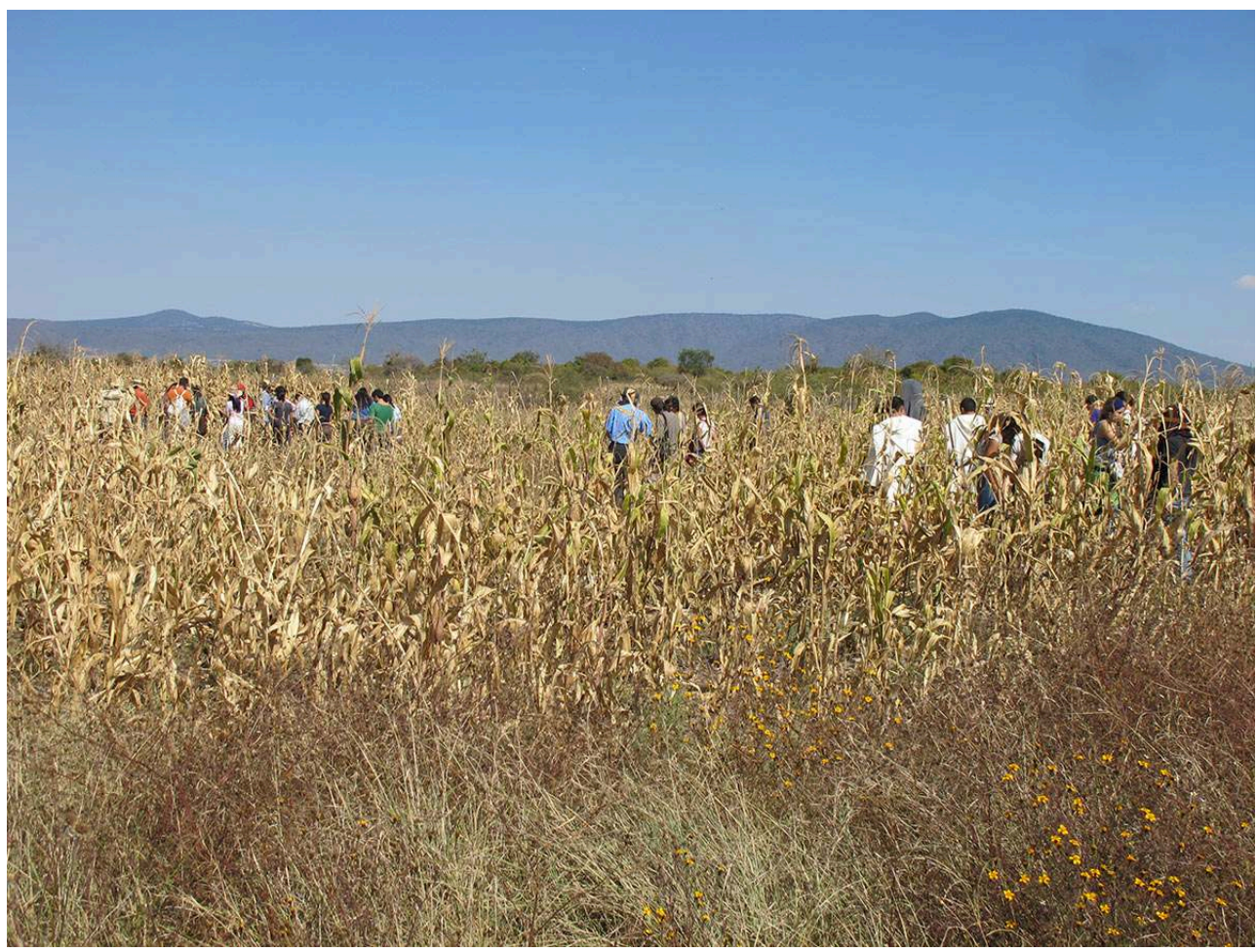

(C) S. Bahuchet 
Figure 26 : Affiche ETB-MX-SB-2010B-018

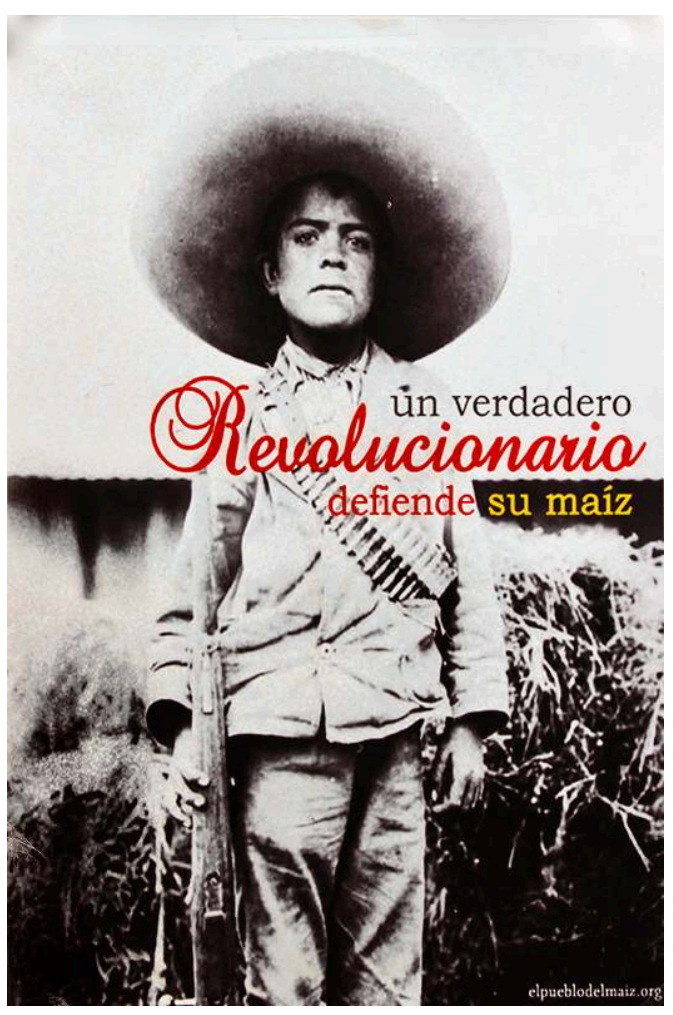

(c) elpueblodelmaiz.org

- Ixtlahuacán de los Membrillo (Jalisco)

- Créé par le réseau RASA. "Un vrai Révolutionnaire défend son maïs », basé sur une photo historique de la Révolution de 1910.

-21/11/2010; collecteur Serge Bahuchet

- $33 \times 47 \mathrm{~cm}$

- ETB-MX-SB-2010B-018

\section{L'agriculture}


Figure 27 : Maïs de plaine et maïs de coteau (Champs de Don Roberto, Chiquilitzli, Chiquilistlán, Jalisco, 18/8/2017)

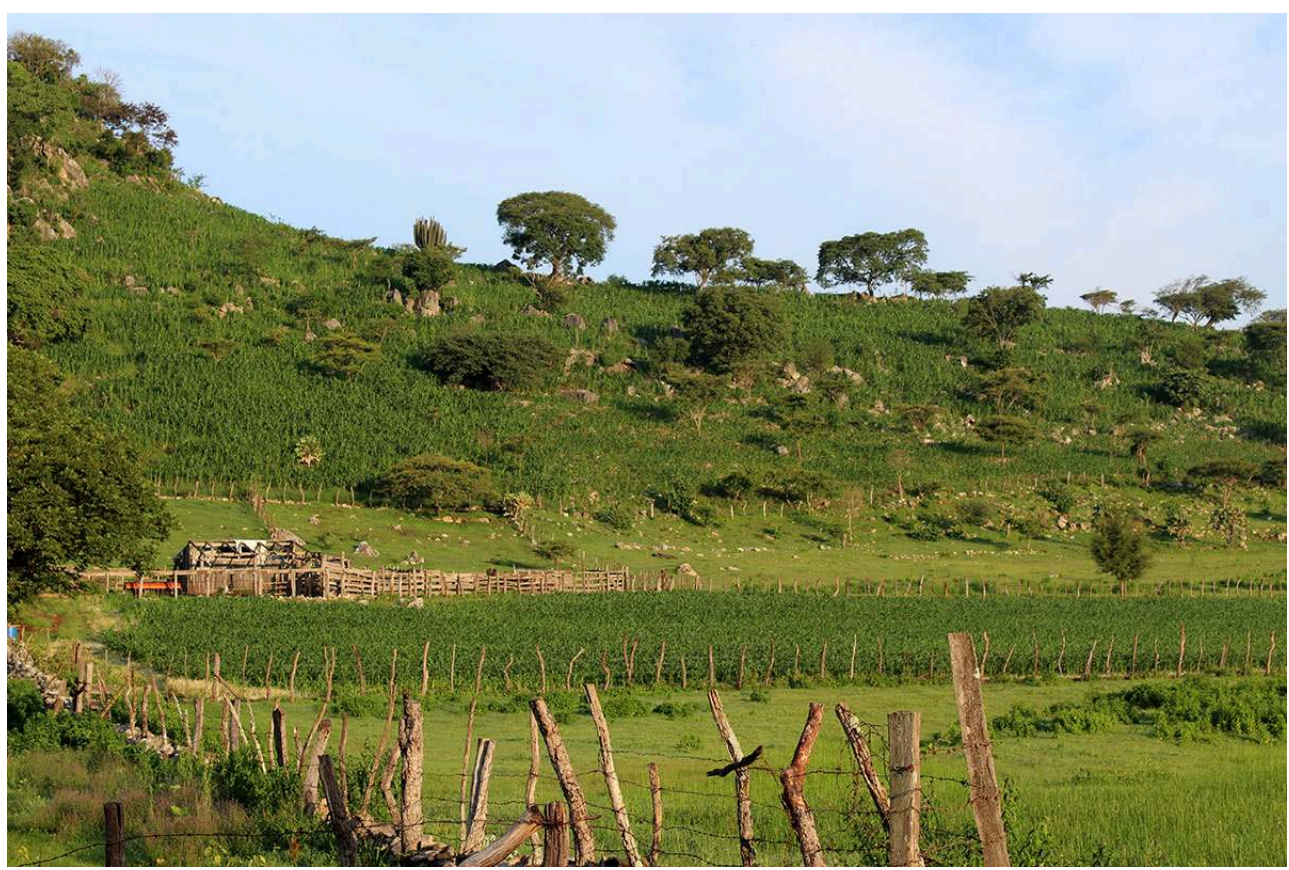

Dans la plaine, au premier plan, les maïs améliorés destinés à la nourriture animale et à la vente. Sur les coteaux pierreux, les coamiles avec les maïs criollos destinés à l'alimentation humaine.

(c) S. Bahuchet

Deux modes de culture contrastés du maïs coexistent au Mexique, auxquels correspondent des outils différents (Figure 27). Dans les vallées, les variétés modernes, améliorées, sont cultivées intensivement avec des machines agricoles, charrues attelées ou tracteurs, et des intrants, en vue de leur commercialisation.

Sur les coteaux et les pentes inaccessibles aux machines, ou même dans les plaines au sol très caillouteux, c'est la culture à la main qui est d'usage. Y sont semées les variétés locales, « natives » (nativas) ou " créoles » (criollas), dans des parcelles de taille limitées, qui fournissent l'alimentation de la famille. Ces lieux de culture sont nommés coamiles ${ }^{4}$ ou bien milpa, et le maïs est souvent associé à d'autres plantes avec qui il entretient une relation d'entraide, principalement des haricots et des courges : les haricots prennent appui sur la tige du maïs, et les grandes feuilles des courges couvriront le sol et maintiendront son humidité (Figure 28). Les variétés de maïs y sont mélangées. Ayant des rythmes de croissance différents et des capacités variées de résistance aux maladies ou aux aléas climatiques, ce mélange augmente la productivité du champ. 
Figure 28 : Association des trois plantes dans la milpa de Don Miguel (1/8/2017, Chiquilistlàn, Jalisco)

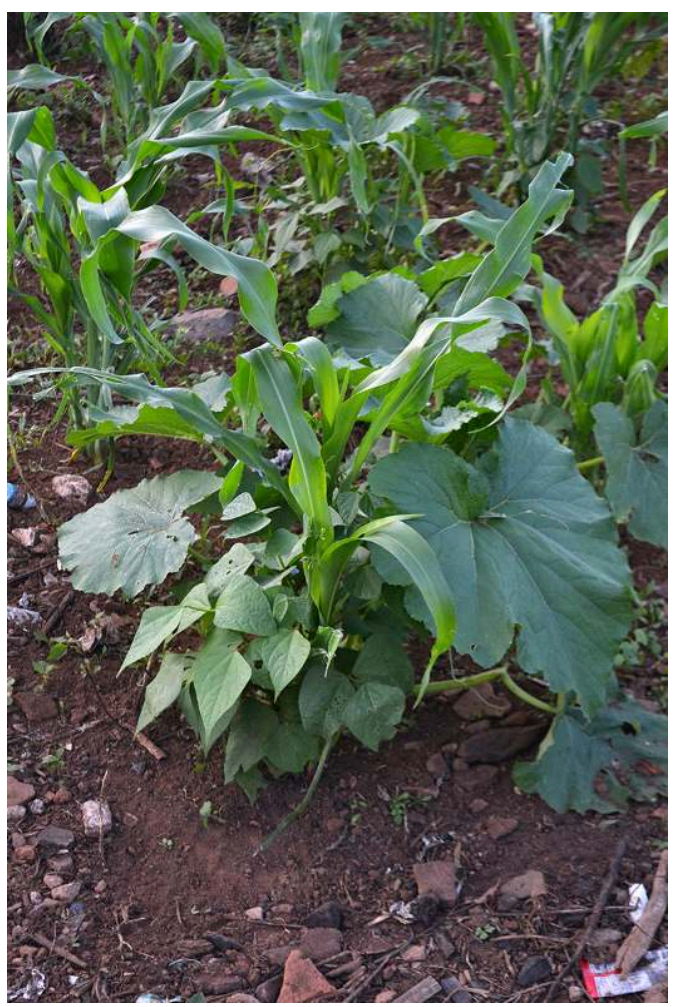

Pousses de haricots (Phaseolus vulgaris) et de courge (Cucurbita pepo), au pied de deux tiges de maïs (Zea mays)

(C) P. Rameau

40 Généralement des jardins sont aménagés dans le village, à côté de l'habitation, ce sont les solares. On y plante des variétés locales de maïs criollos ou nativos, des haricots, des courges, des piments, des Physalis ixocarpa (Solanaceae, tomatillos), tous ingrédients que l'on doit avoir sous la main pour la cuisine quotidienne (Figures 29, 62). 
Figure 29 - Les solares à Chiquilistlán (Jalisco), Juillet 2017

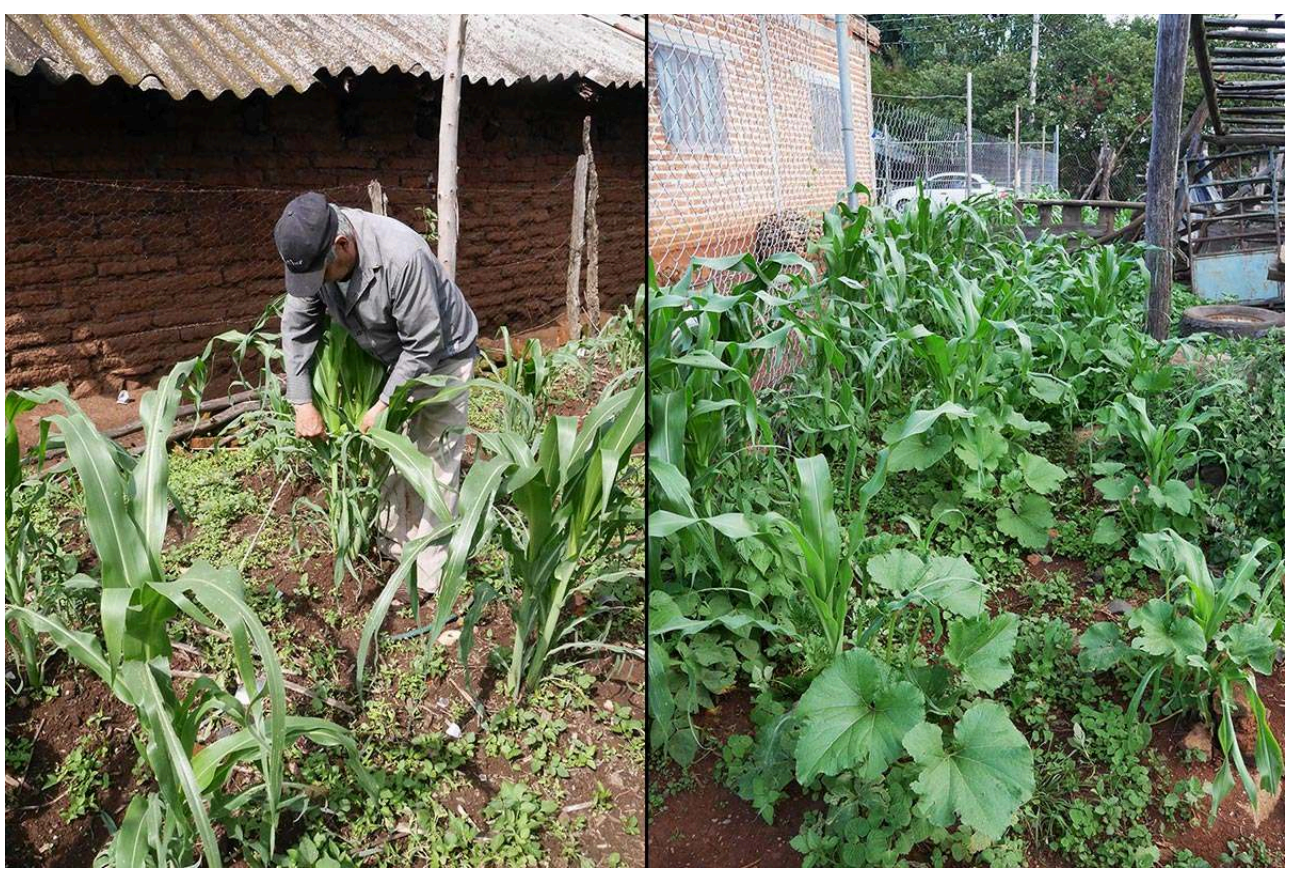

Solares de Lauro Cordena (qui cueille des feuilles de maïs) et de Luis Medina.

(c) S. Bahuchet

\section{La journée au champ}

\section{L'équipement du paysan}

41 Le paysan se rend quasi-quotidiennement dans ses parcelles cultivées, souvent en montant une mule (Figures 30,31) et accompagné de ses chiens. Il porte sa machette, une gourde d'eau, une musette à l'épaule et se protège du soleil par le fameux sombrero mexicain! 
Figure 30 : «Subiendo al coamil », Don Lauro et ses fils partent aux champs (9/9/2017, Chiquilistlàn, Jalisco)

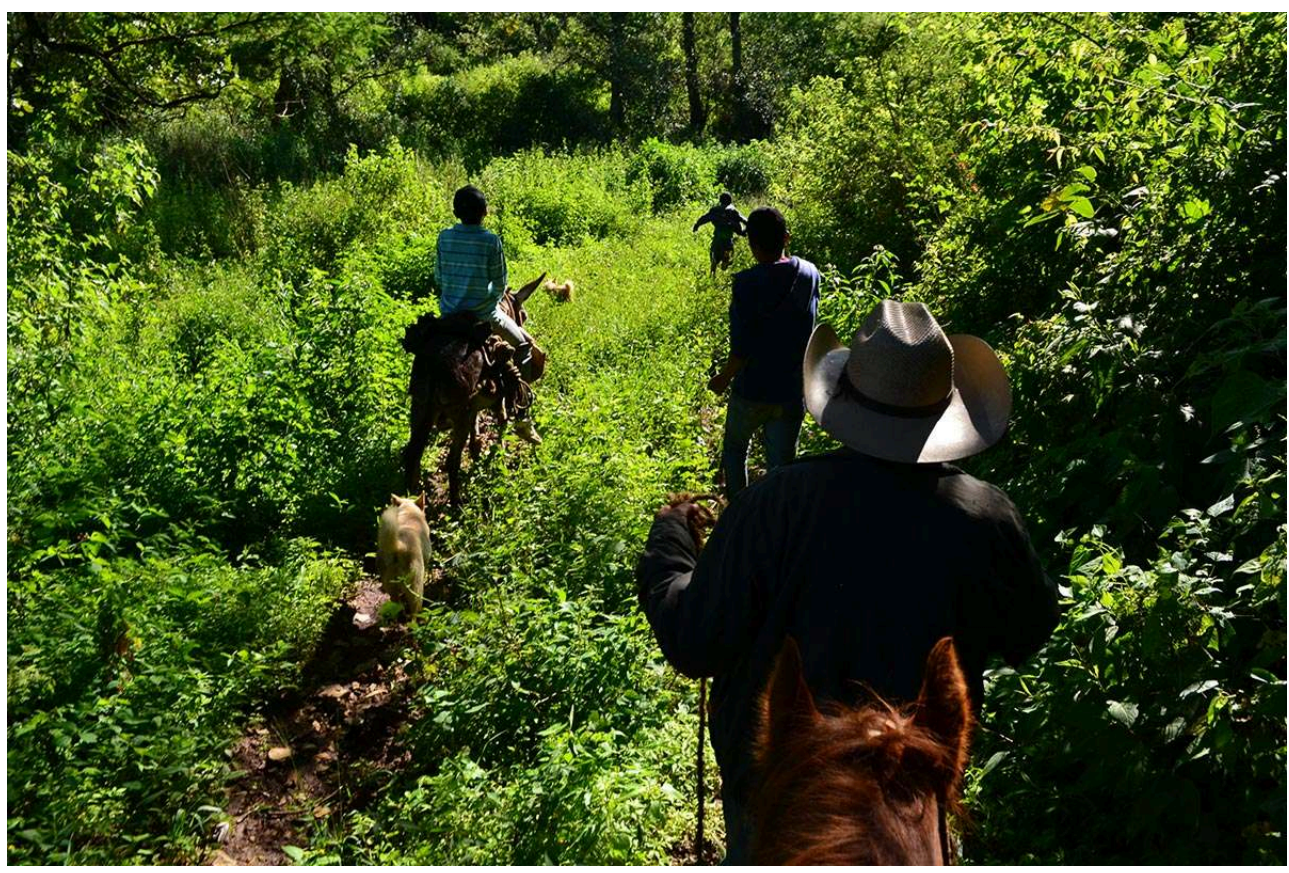

(C) P. Rameau

Figure 31 a et $b$ : Selle de mule harnachée, avec la machette dans son fourreau, exposée dans la Galerie de l'Homme ETB-MX-AB-2014-001 (la selle), ETB-MX-SB-2014-070 (la machette), ETB-MXPR-2014-101 (fourreau)
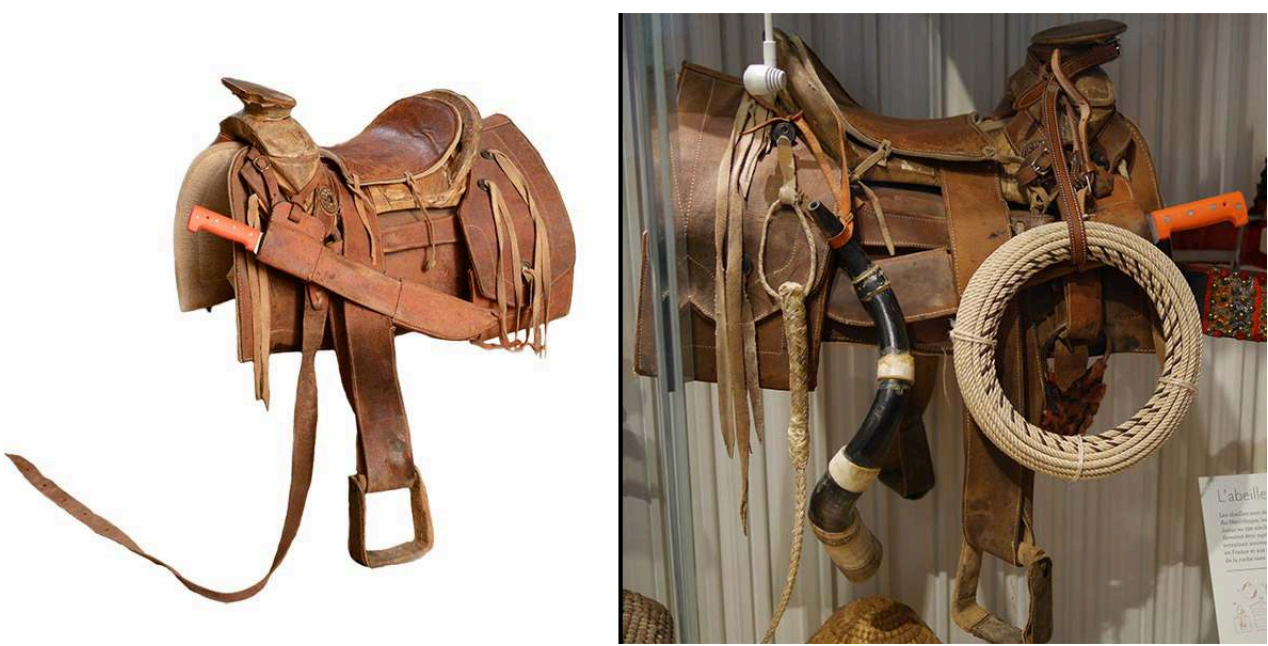

Selle provenant de San Miguel et machette de Mascota (Jalisco), fourreau provenant de Oaxaca. (c) J.-C. Domenech-MNHN, (vitrine) (c) S. Bahuchet

42 5) Machette dans son fourreau (machete y frenda) 
Figure 32 : Machette dans son fourreau ETB-MX-SB-2012-23a et b

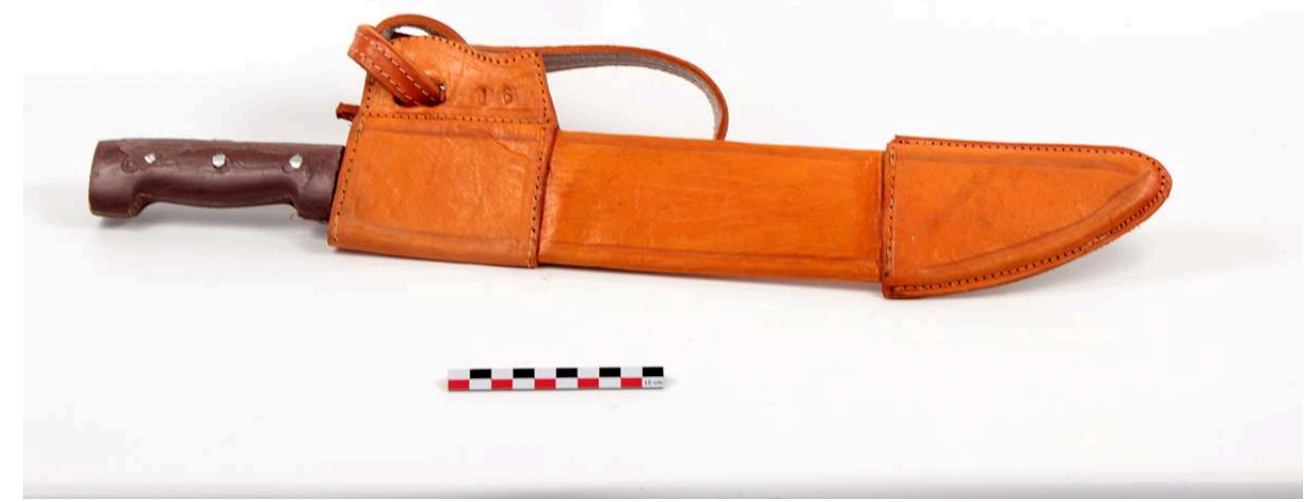

(c) P. Rameau

43 - Guadalajara (Jalisco), achat en quincaillerie

- Lame en métal, manche en matière plastique, fourreau en cuir

- 2/4/2012 ; collecteur Serge Bahuchet

- $55 \times 13 \mathrm{~cm}$

- ETB-MX-SB-2012-023a et b

Figure 33 : Mule harnachée avec la machette dans un fourreau de fabrication domestique, en bâche cousue (15/4/2010, Cuzalapa, Jalisco)

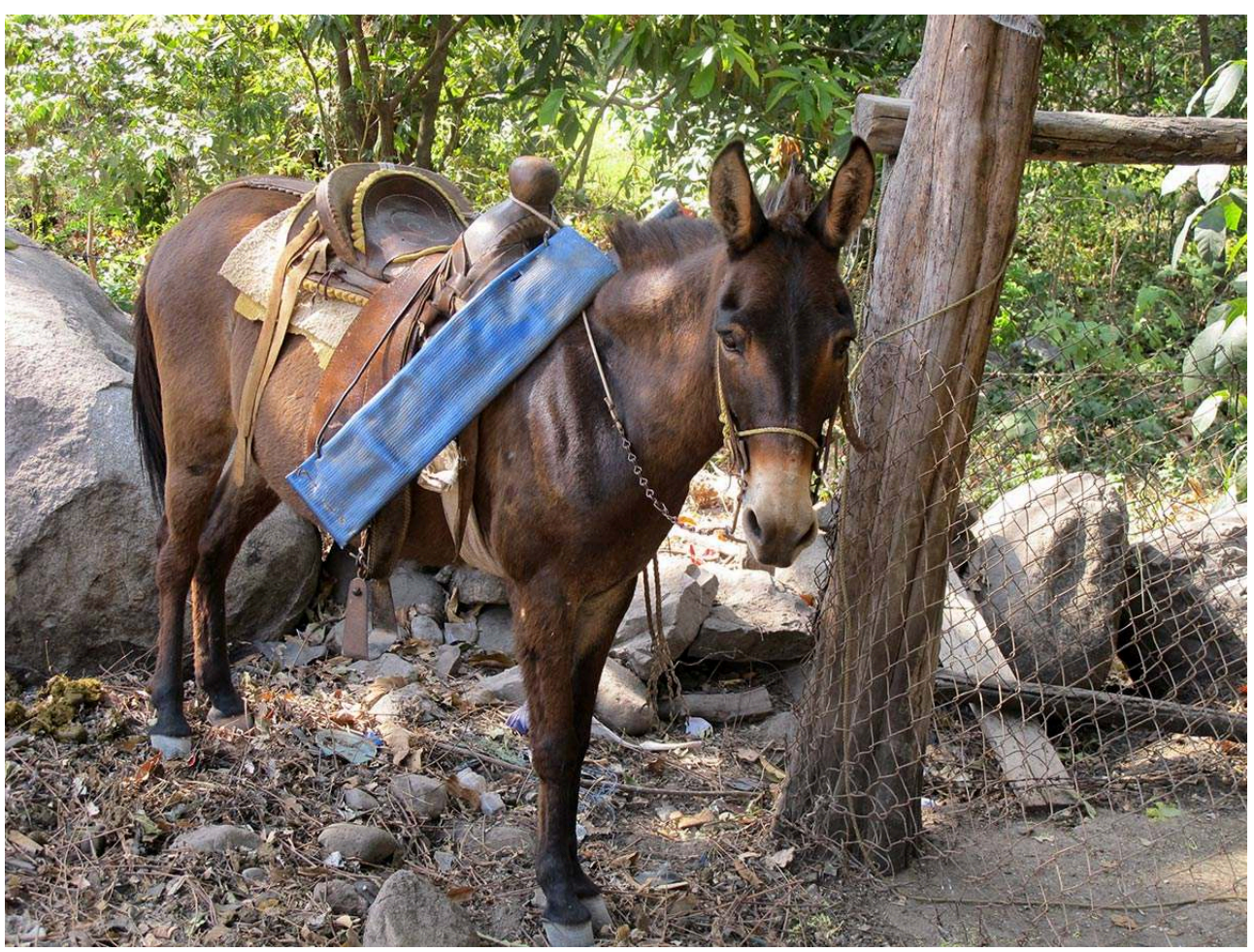

(c) S. Bahuchet

6) Fourreaux de machette (funda de piel) 

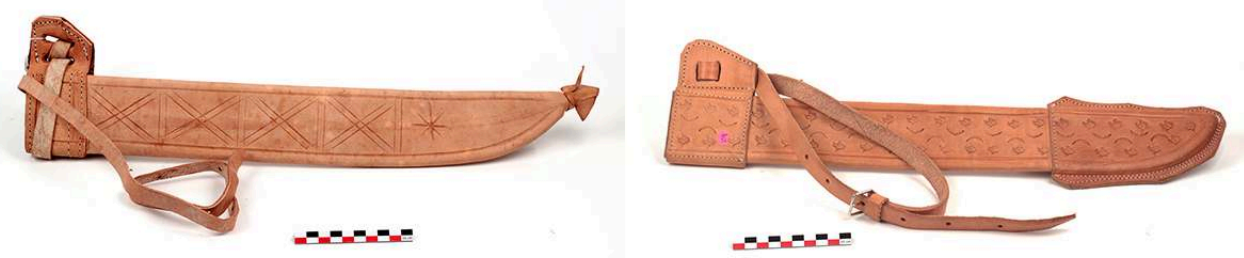

En cuir cousu, fabriqués localement, par les bourreliers, fabricants de selles

À gauche : ETB-MX-SB-2018-019, Quincaillerie dans le quartier Merced, CDMX, 19/4/2018, collecteur Serge Bahuchet

À droite : ETB-MX-PR-2014-177, San Cristobal (Chiapas), fabriqué à Comitan de Dominguez,

7/12/2014, collectrice Pauline Rameau

(c) S. Bahuchet

\section{Les récipients pour porter l'eau aux champs}

L'eau à boire est contenue dans des gourdes en fruits secs de calebasse Lagenaria siceraria (Molina) Standl. (Cucurbitaceae, Figure 35), fréquemment pourvus d'une partie renflée et d'un col plus ou moins ventru, en forme de bouteille; en général on le bouche avec un morceau de rafle de maïs. Ces gourdes peuvent être nues, avec une simple ficelle pour les porter ou les suspendre, ou enveloppées dans une résille, qui les protège contre les chocs, ce fruit étant très cassant. Beaucoup cultivent des gourdes dans leur jardin, mais on en trouve dans tous les commerces (Figure 36).

Figure 35 : Lagenaria cultivées au Jardin des Plantes en 2019

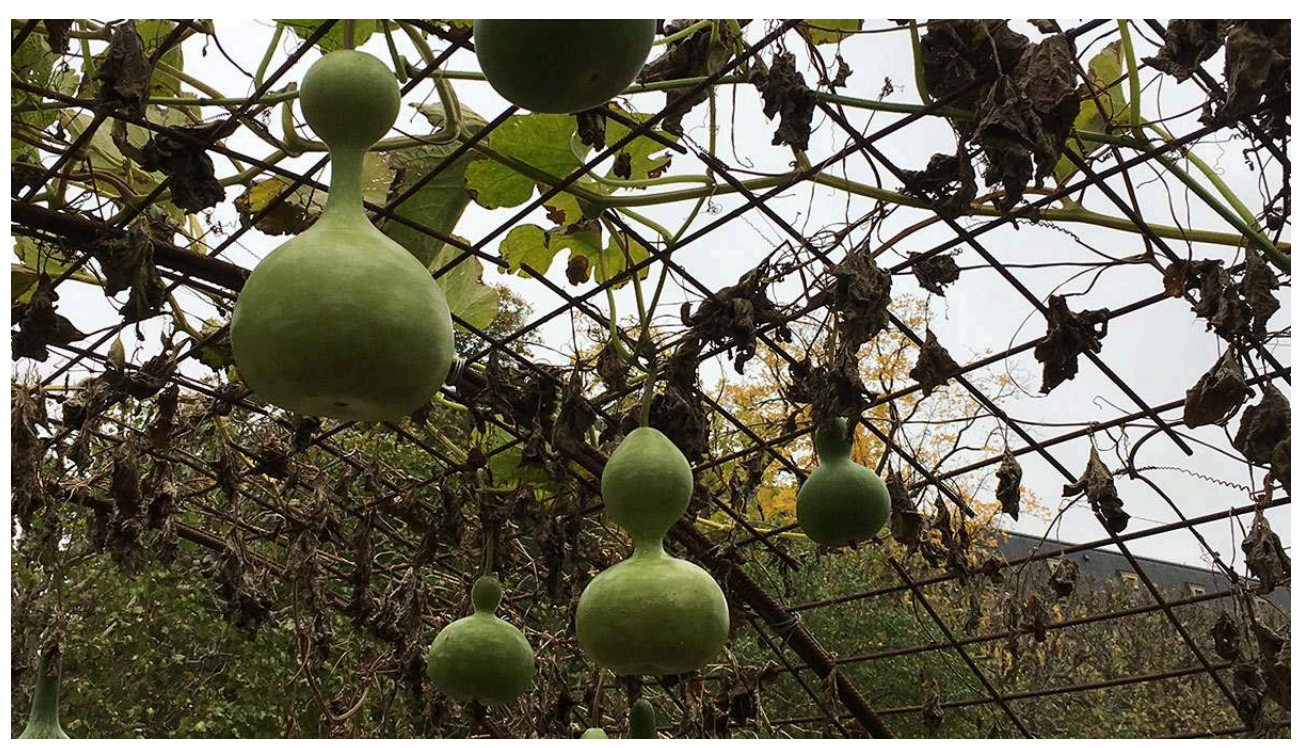

(c) S. Bahuchet 
Figure 36 : Gourdes au marché de Colima (21/4/2010)

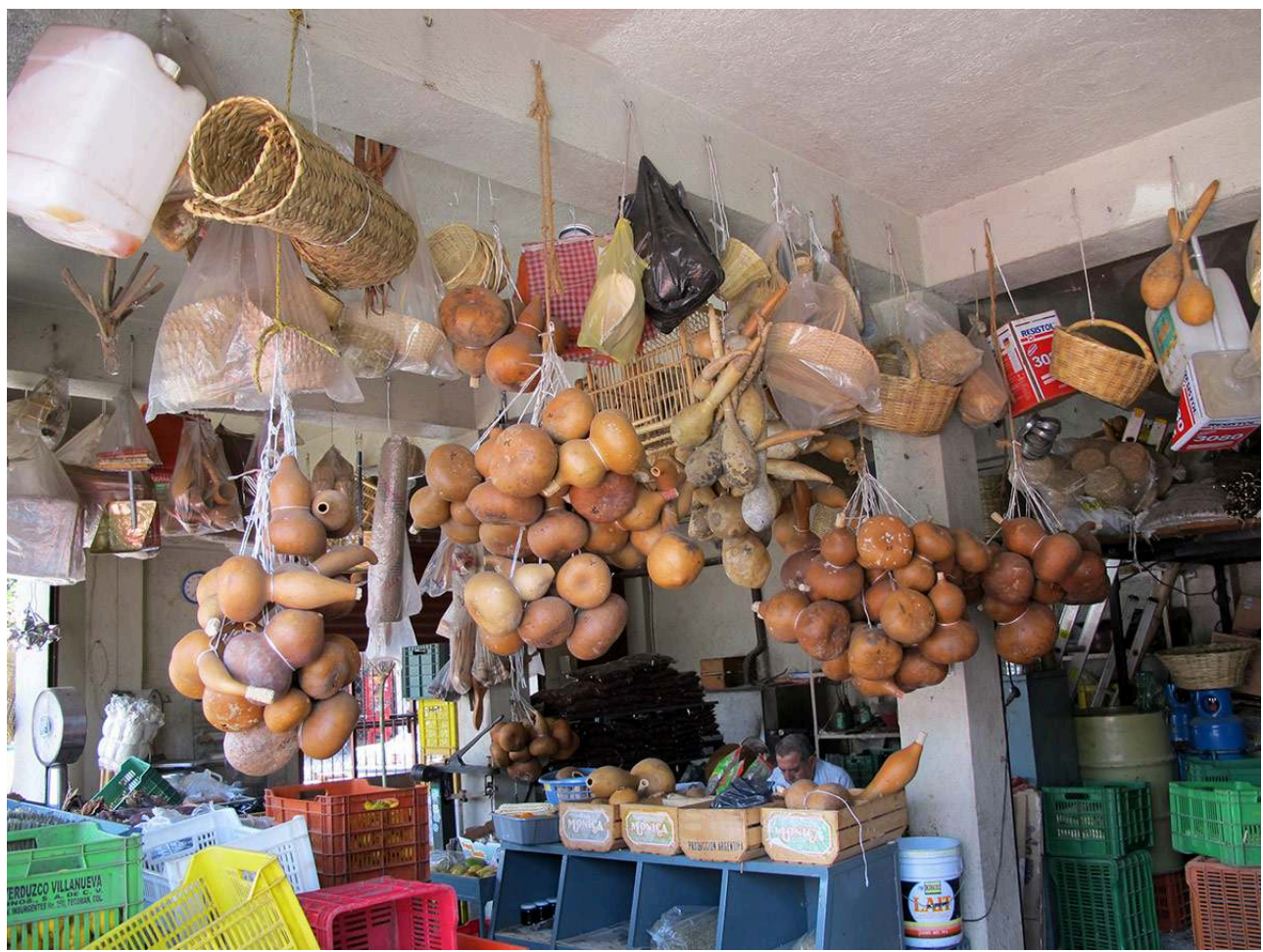

(c) S. Bahuchet

7) Gourdes nues

Figure 37 : Gourdes nues ETB-MX-SB-2015-003 et ETB-MX-SB-2014-018

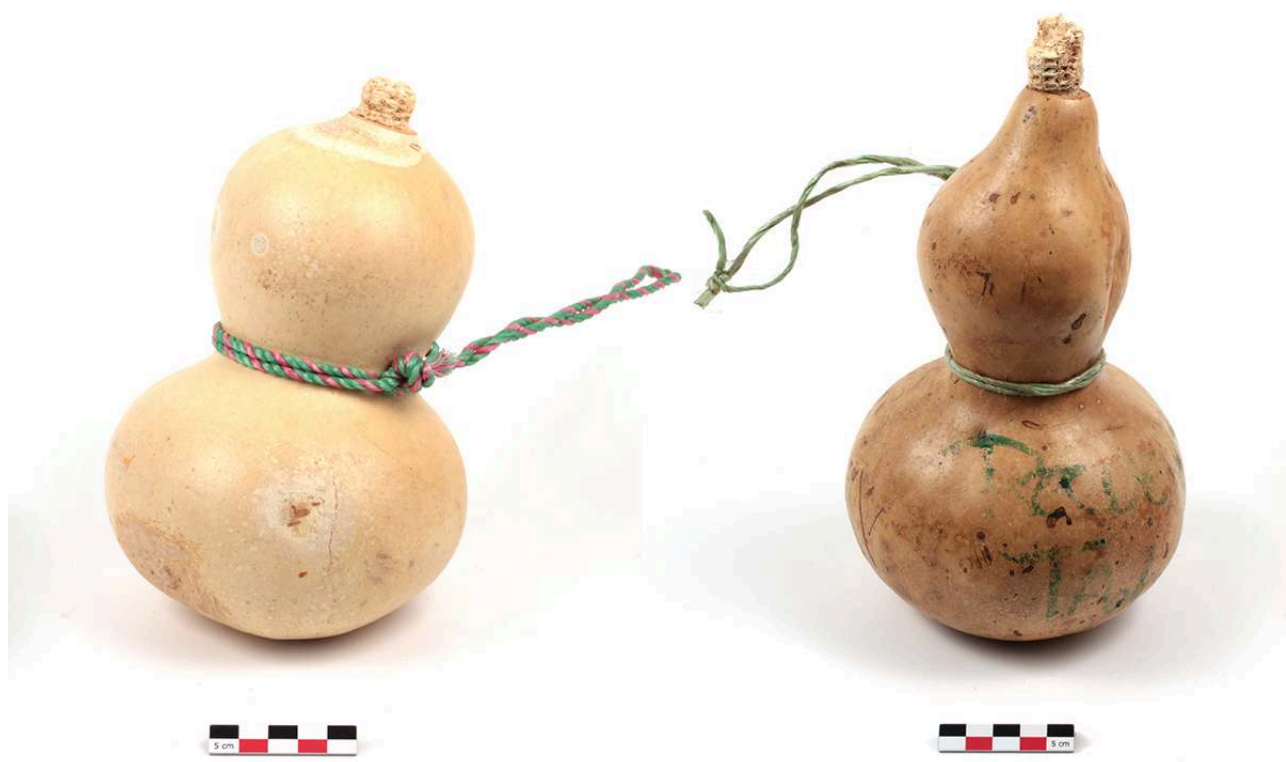

À gauche : ETB-MX-SB-2015-003, Xoxocotle (Morelos), $\varnothing 13, \mathrm{H} 20 \mathrm{~cm}, 24 / 8 / 2015$, achat en jardinerie À droite : ETB-MX-SB-2014-018, Talpa de Allende (Jalisco), Ø 12, H 22 cm, 17/4/2014

(c) P. Rameau 
Figure 38 : Gourdes protégées ETB-MX-SB-2010A-024 et ETB-MX-SBPR-2017-081
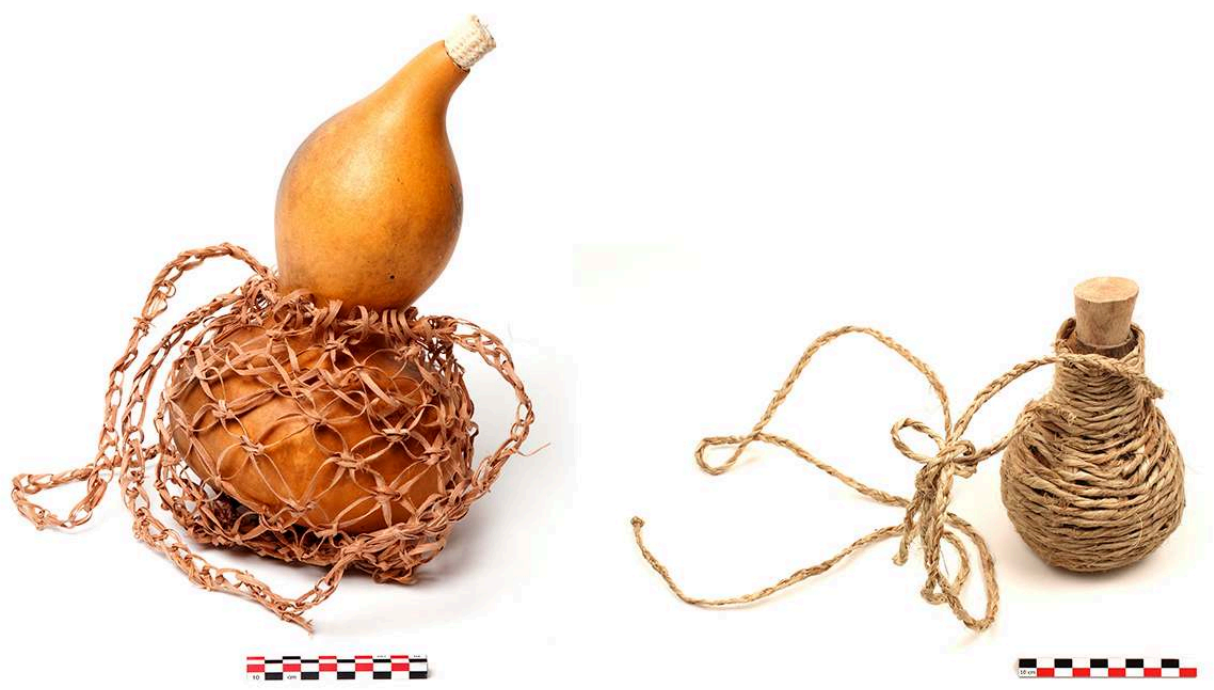

À gauche : ETB-MX-SB-2010A-024, Gourde et son filet en fibres d'agave, bouchon en rafle de maïs, Cuzalapa (Jalisco), $35 \mathrm{~cm} \varnothing 16,15 / 4 / 2010$

À droite : ETB-MX-SBPR-2017-081, Gourde protégée (at tecomate) par une corde en écorce de jonote (Heliocarpus donnelsmithii, Malvaceae), bouchon de bois, Cuetzatlan (Puebla), Nahua, $\varnothing 10 \mathrm{H} 17$, 22/9/2017

(C) P. Rameau

9) Bouteille pour l'eau 


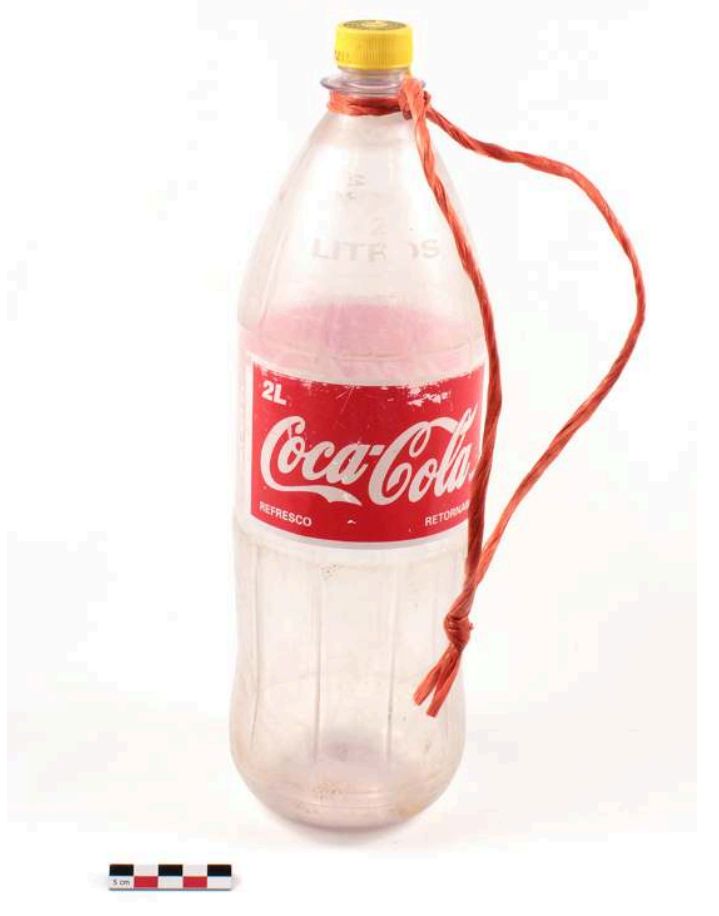

(C) P. Rameau

49 - Chiquilistlán (Jalisco)

- Matière plastique, boutique rigide de 21 . ; s'accroche à la selle de la mule

-17/4/2018 ; collectrice Pauline Rameau, don Lauro Cordena

- H $34 ø 9,5 \mathrm{~cm}$

- ETB-MX-PR-2018-006

\section{Les chapeaux (sombreros)}

Les sombreros sont disponibles dans de nombreuses boutiques comme sur les marchés ; ils peuvent aussi être confectionnés par certains vanniers spécialisés, qui sont en même temps cultivateurs (Figure 41). Les larges chapeaux, tressés (Figures 40,42) ou cousus (Figure 43), protègent le paysan du soleil, et quelquefois d'une averse, lors des longues journées dans les champs.

51 10) Chapeau (sombrero de otate) 


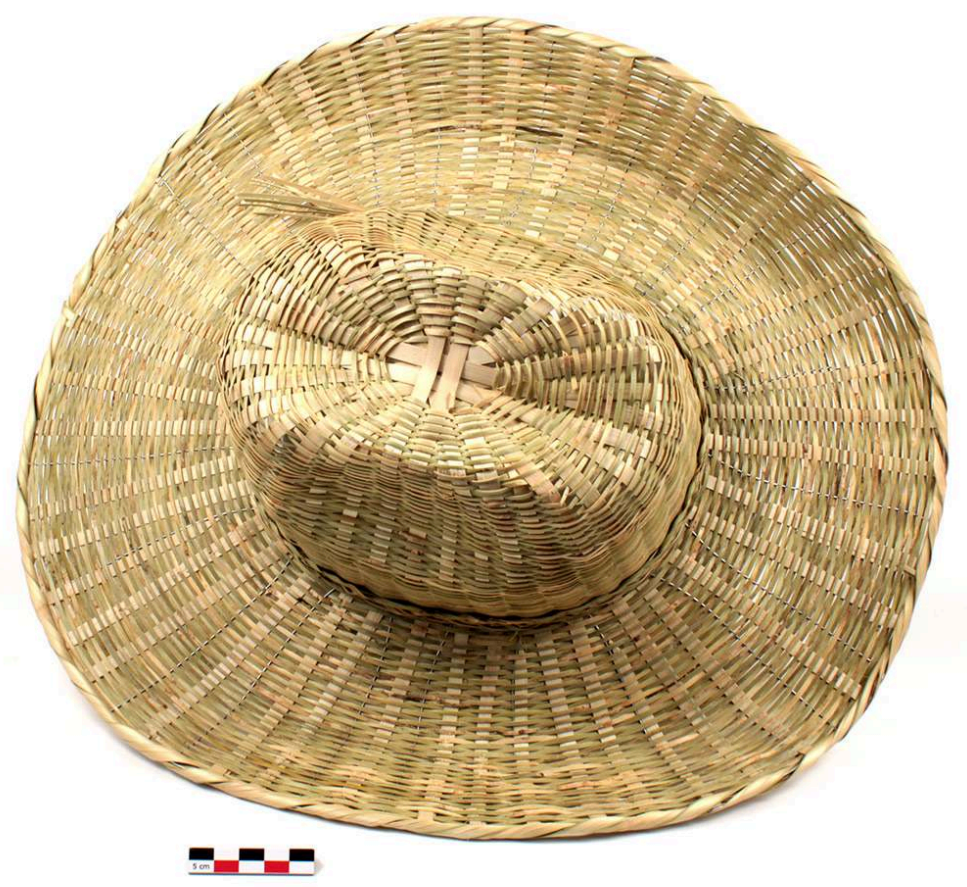

(c) P. Rameau

52 - Chiquilistlán (Jalisco)

- Bambou otate (Otatea accuminata, Poaceae) sur armature de fer, à l'intérieur une bande de simili cuir. Confectionné par Lauro Cordena

-17/4/2018 ; collectrice Pauline Rameau, don de Lauro Cordena

- $42 \times 38 \times 15 \mathrm{~cm}$

•ETB-MX-PR-2018-007 
Figure 41 : Atelier de tressage des chapeaux par Don Lauro, qui en commence un nouveau, et son fils, qui affine les brins d'otate (Chiquilistlán, Jalisco, 29/8/2017)

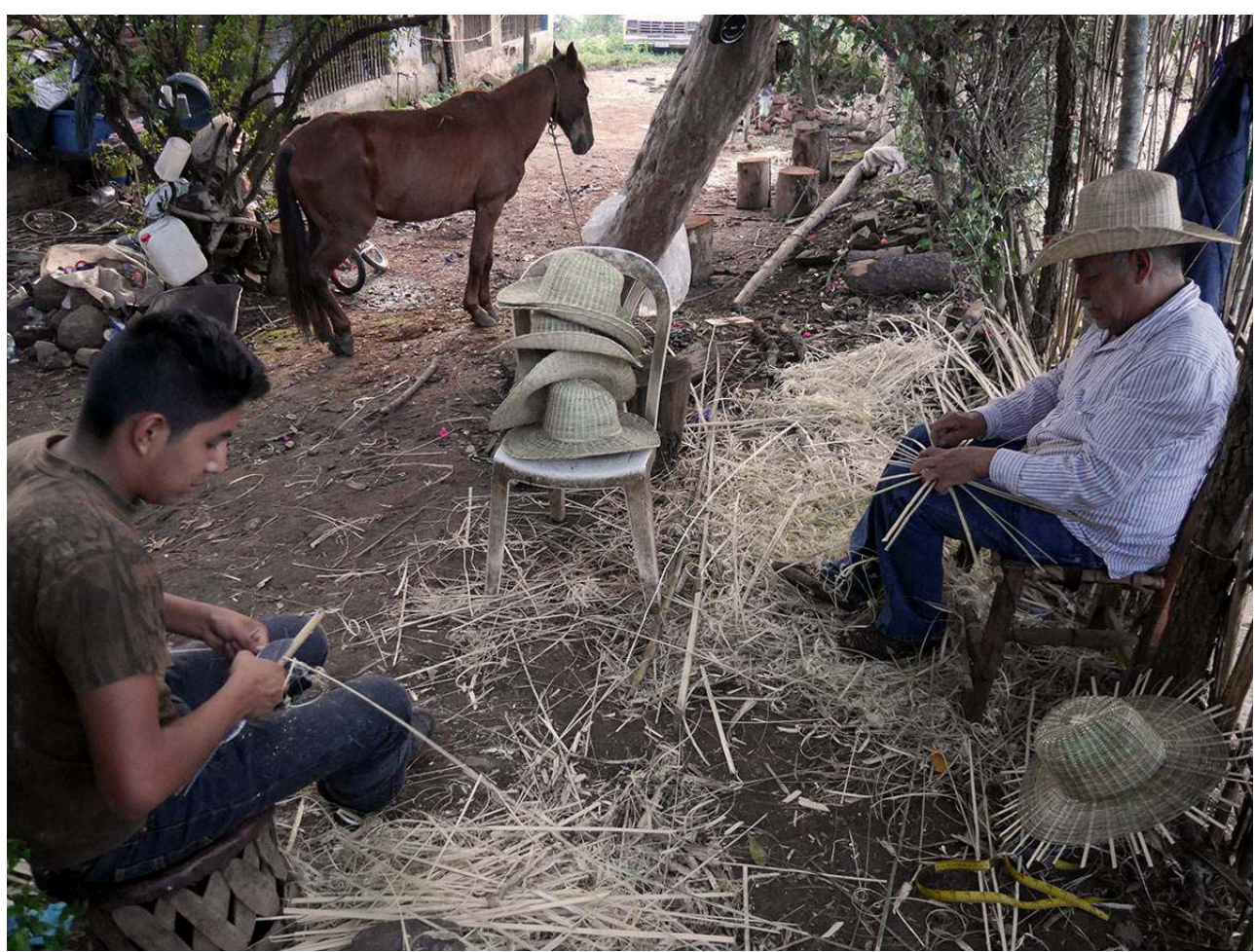

(c) P. Rameau

11) Chapeau de soleil 


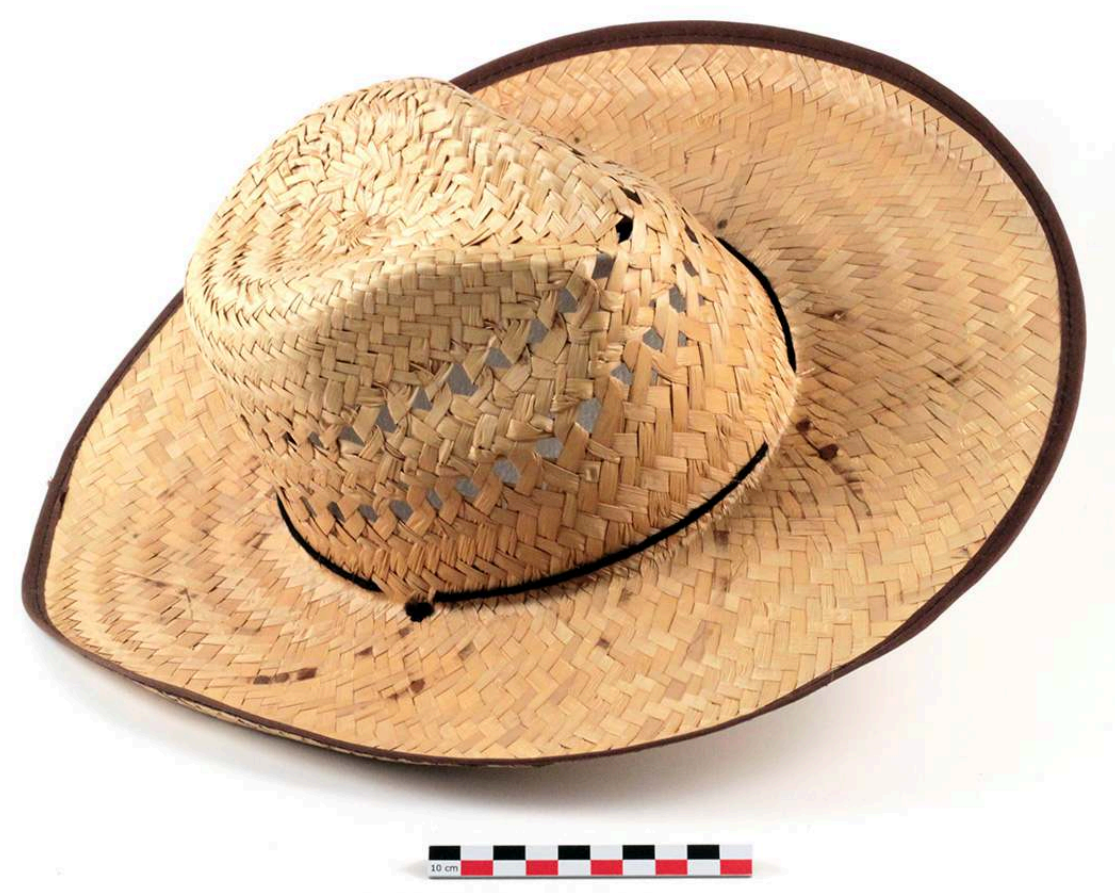

(C) S. Bahuchet

54 - Teotihuacan (État de Mexico)

- Palme tressée (probablement Brahea dulcis, Arecaceae), cordon en laine

-18/4/2018; collecteur Serge Bahuchet

- $45 \times 32 \times 14 \mathrm{~cm}$

- ETB-MX-SB-2018-016

12) Chapeau (sombrero) 


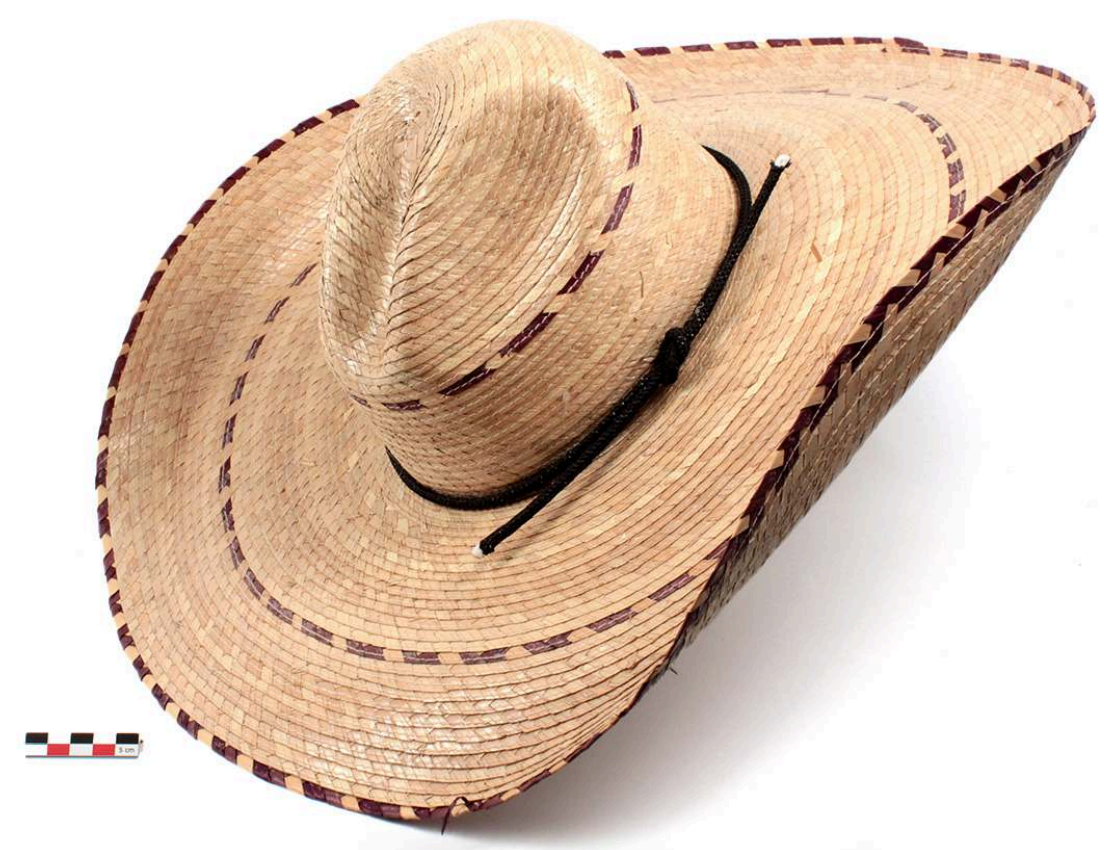

(C) S. Bahuchet

56 - Guadalajara (Jalisco)

- En palme cousue

- Avril 2010 ; collecteurs Françoise Aubaile et Serge Bahuchet

- 49 x 40 x $15 \mathrm{~cm}$

- ETB-MX-SB-2010A-041

\section{Les manteaux de pluie}

57 En sus du couvre-chef, le costume classique du paysan comporte (ou comportait) une cape réalisée avec des folioles de palmier, qui le protège des pluies. Toujours disponibles dans les quincailleries locales (Figure 44), ces manteaux de pluie sont surtout, de nos jours, employés au cours des fêtes votives calendaires, dans les communes (Figure 45). 
Figure 44 : Démonstration du port du manteau de pluie par le quincailler lui-même $(23 / 8 / 2015$, Zacatlán, Puebla)

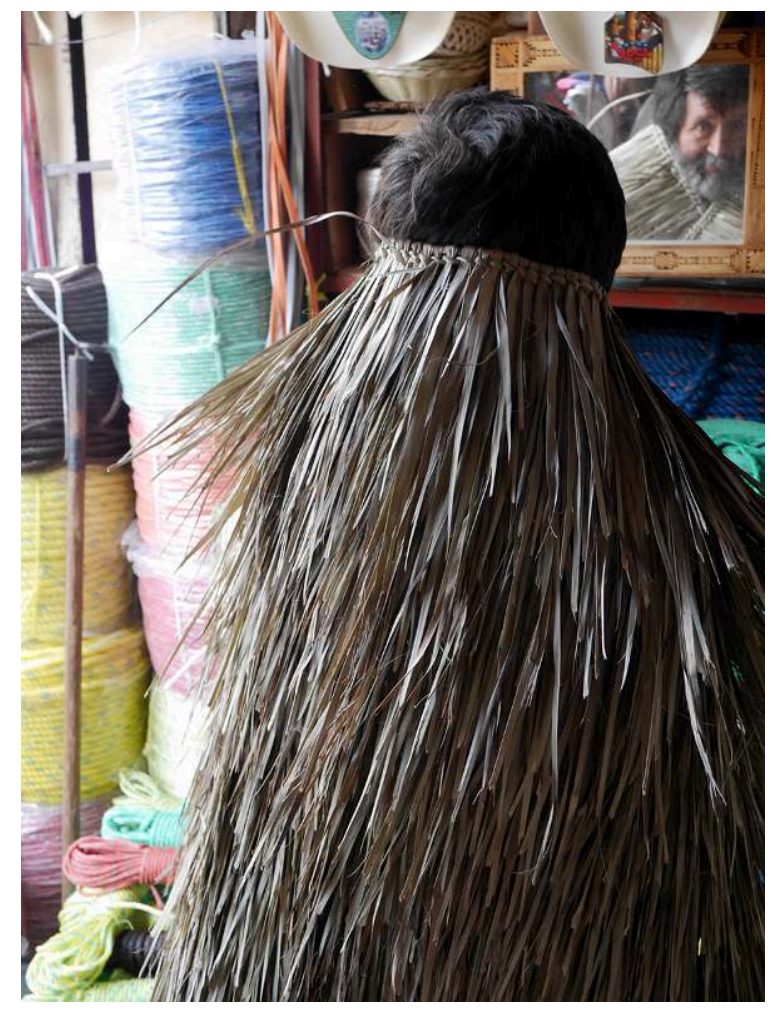

(C) S. Bahuchet

Figure 45 : Danses avec les manteaux de pluie au cours des fêtes du Corpus Christi à Cherán (Michoacán, 14/6/2017)

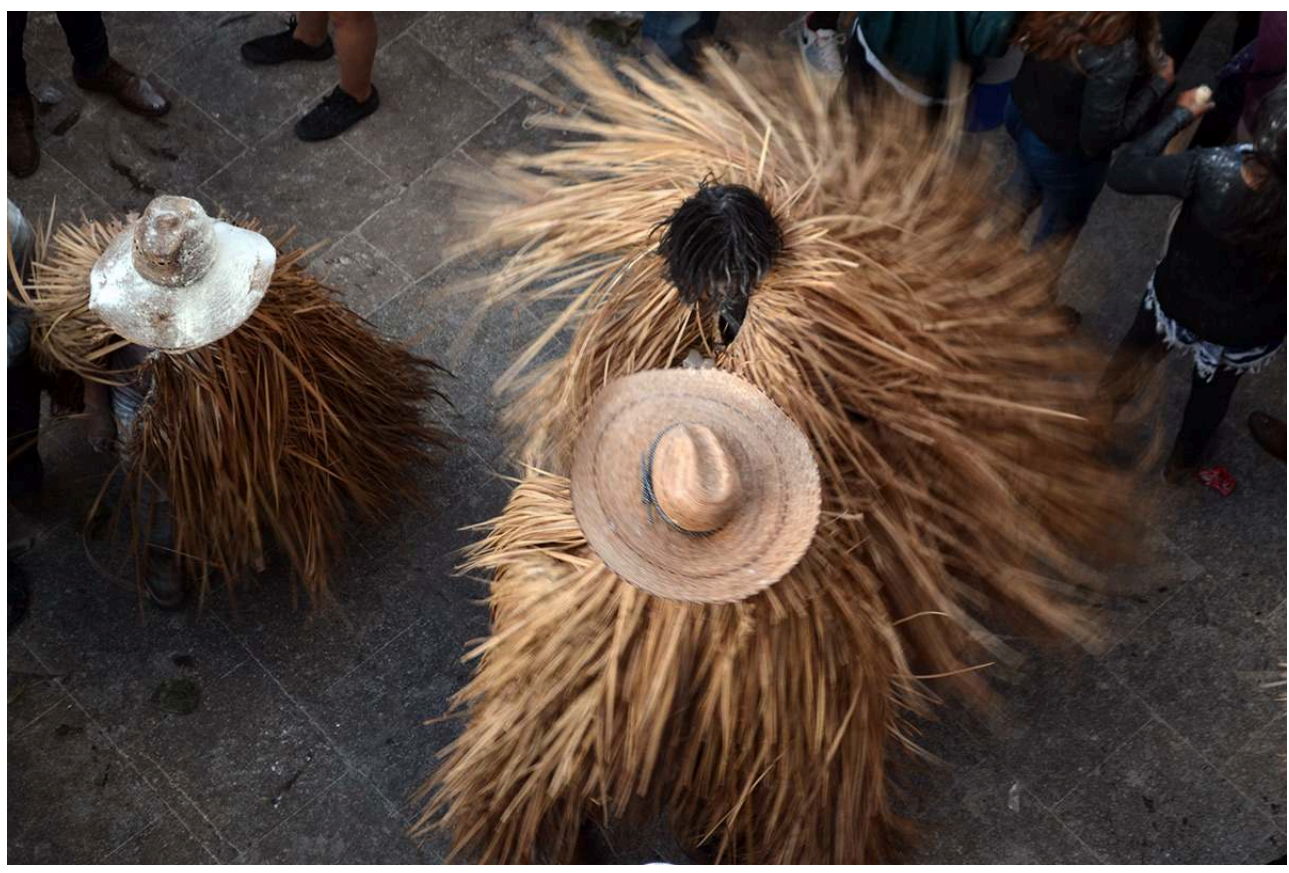

(c) P. Rameau 


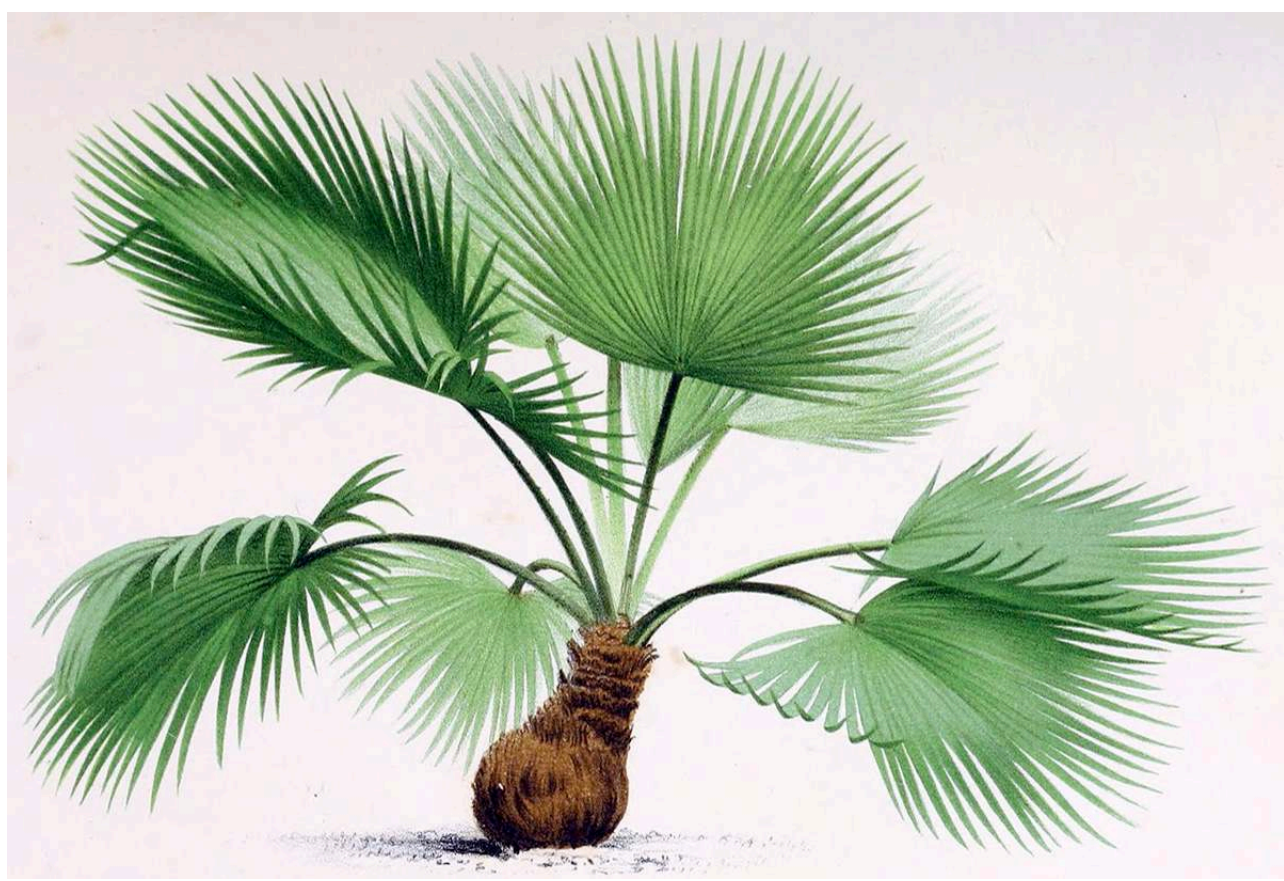

Deux palmiers mexicains sont très communément employés pour la vannerie, Brahea dulcis et Sabal mexicana, dont les feuilles présentent un aspect similaire. Les deux espèces peuvent être utilisées pour tresser les capes de pluie.

Extrait de O. de Kerchove, 1878, Les palmiers. Histoire iconographique, planche XXIX

Le nouage est subtil. La première rangée de folioles est accrochée sur une corde. La foliole est repliée sur elle-même autour de la corde, et repasse en boucle par un nœud «en tête d'alouette » (Figure 47). Chaque foliole est divisée en trois brins : l'un part vers l'extérieur pour créer la protection, deux se divisent vers la droite et vers la gauche, pour servir d'accroche aux palmes du rang suivant. Les brins résultant des deux palmes contiguës du rang supérieur sont noués l'un à l'autre pour permettre l'accroche de la foliole suivante, par une boucle. 
Figure 47 : Détail de l'attache des folioles des palmes : nœud d'accroche " tête d'alouette " (sur spécimen ETB-MX-SB-2015-025, § 14)

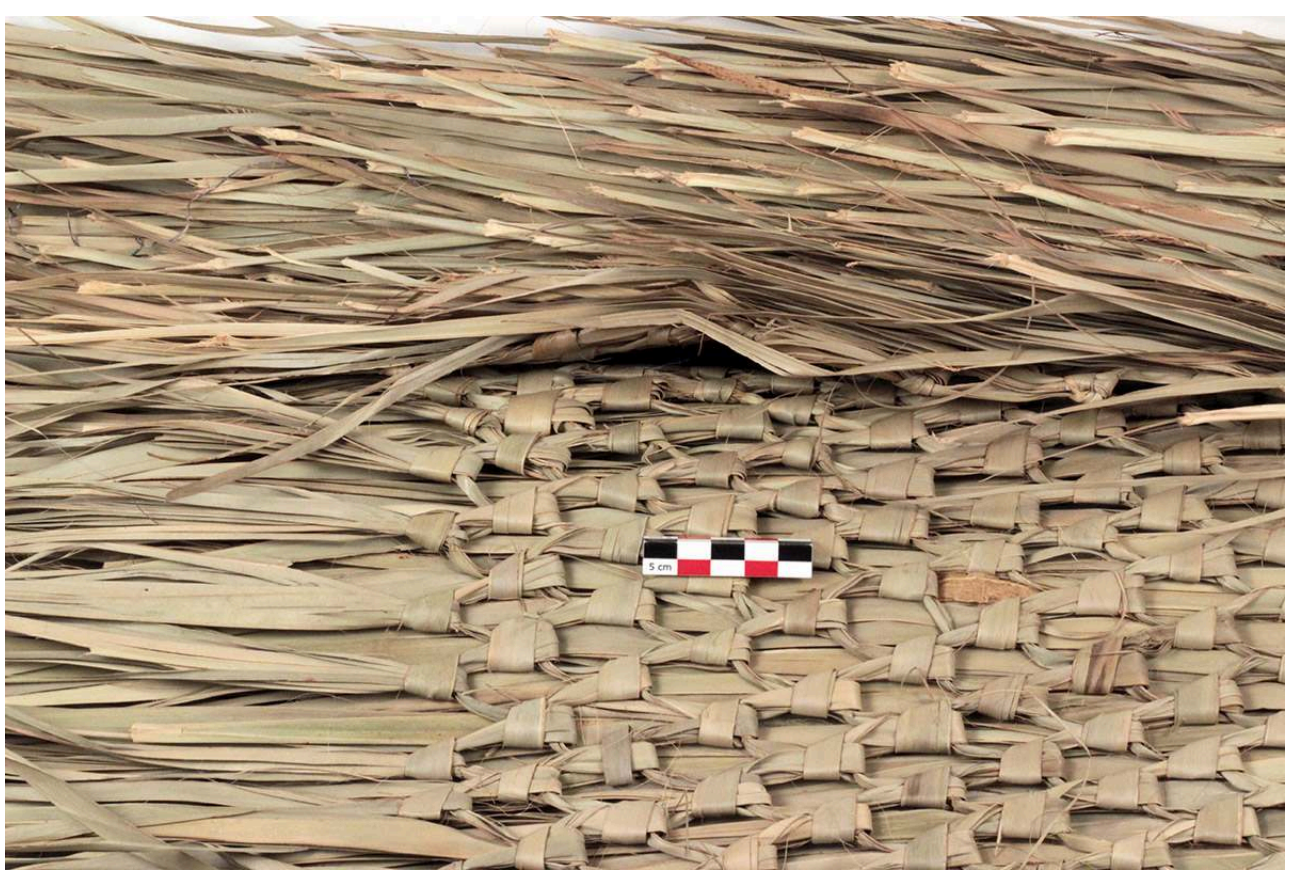

(c) S. Bahuchet

59 13) Manteau de pluie (china)

Figure 48 : Manteau de pluie ETB-MX-SB-2013-035

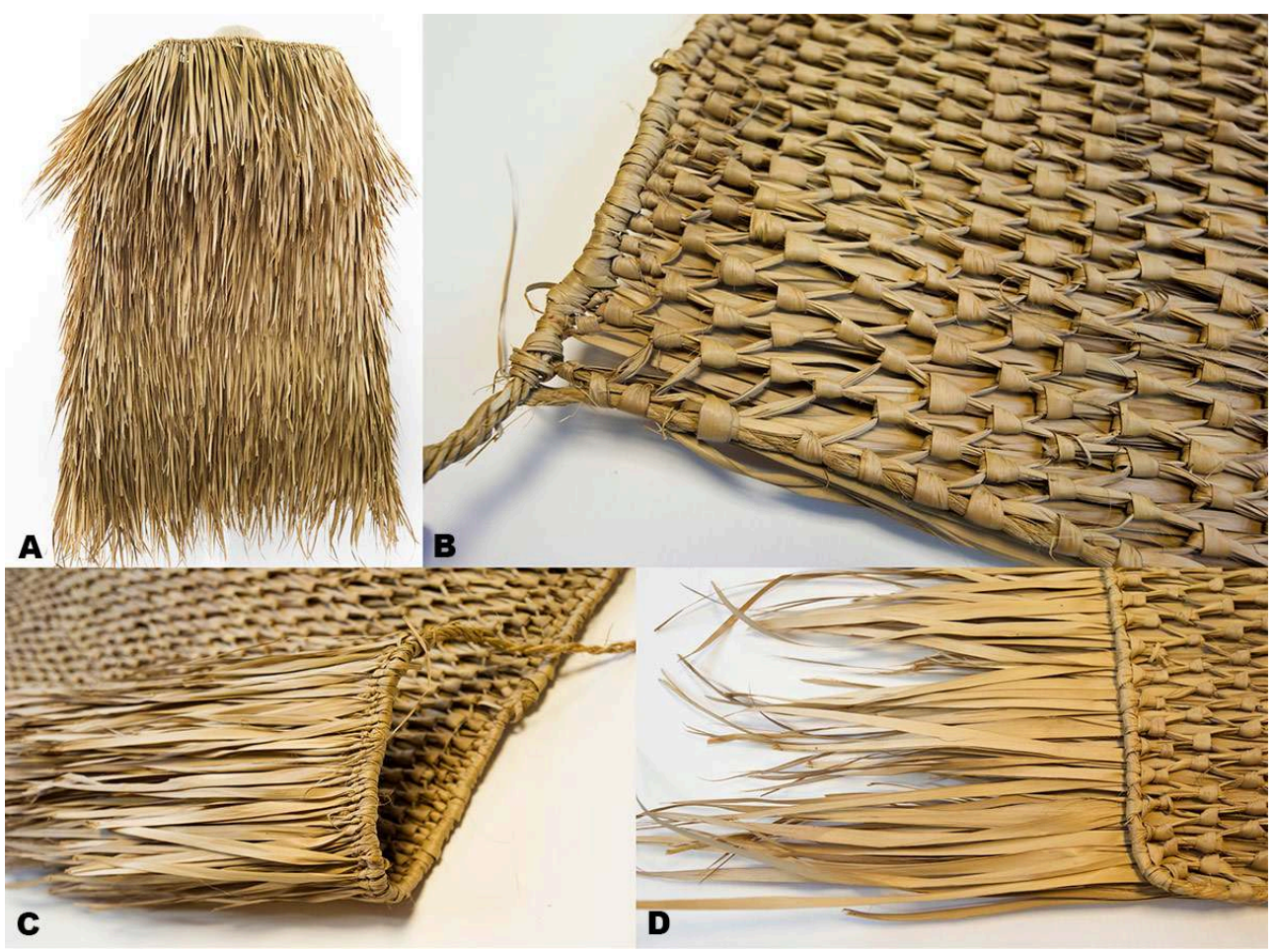

Détails du manteau ETB-MX-SB-2013-035 : B. les nœuds face intérieure, C. la ligne supérieure et D. la ligne inférieure.

(c) N. Idalie 
- Achat à Guadalajara, origine Zacatecas

- En palmes tressées

- 10/4/2013 ; collecteur Serge Bahuchet

- Longueur $93 \mathrm{~cm}$

- ETB-MX-SB-2013-035

61 14) Manteau de pluie (tapizayo)

Figure 49 : Manteau de pluie ETB-MX-SB-2015-025

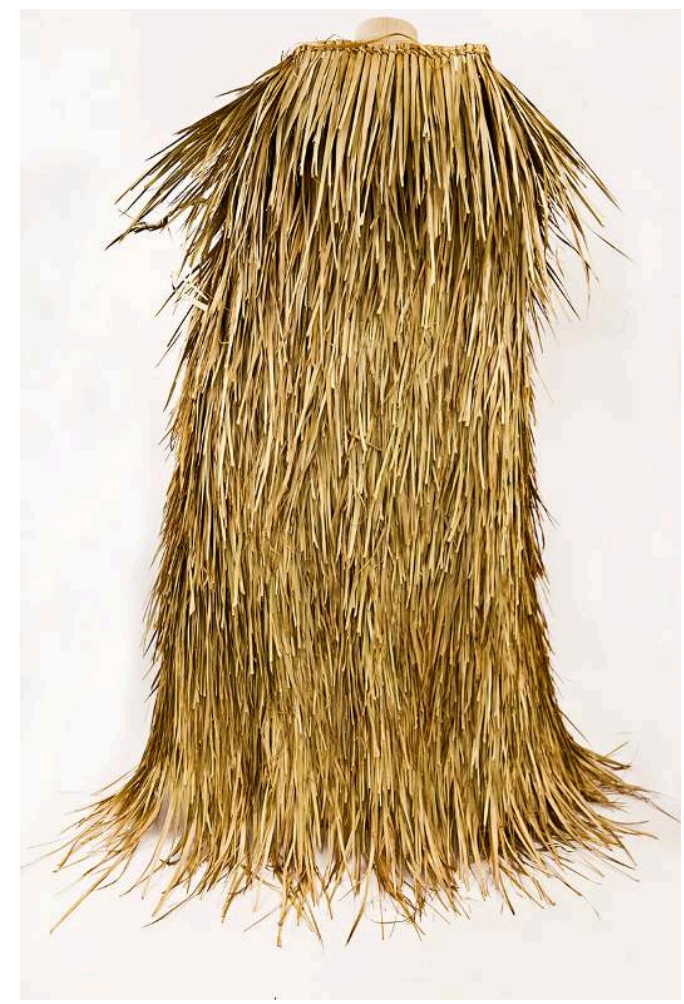

(c) N. Idalie

62 - Zacatlán (Puebla), achat en quincaillerie

- En palmes tressées

- 23/8/2015 ; collecteur Serge Bahuchet

- Longueur $145 \mathrm{~cm}$

- ETB-MX-SB-2015-025

63 15) Manteau de pluie 
Figure 50 : Manteau de pluie nahua MNHN-E-2002.1.37

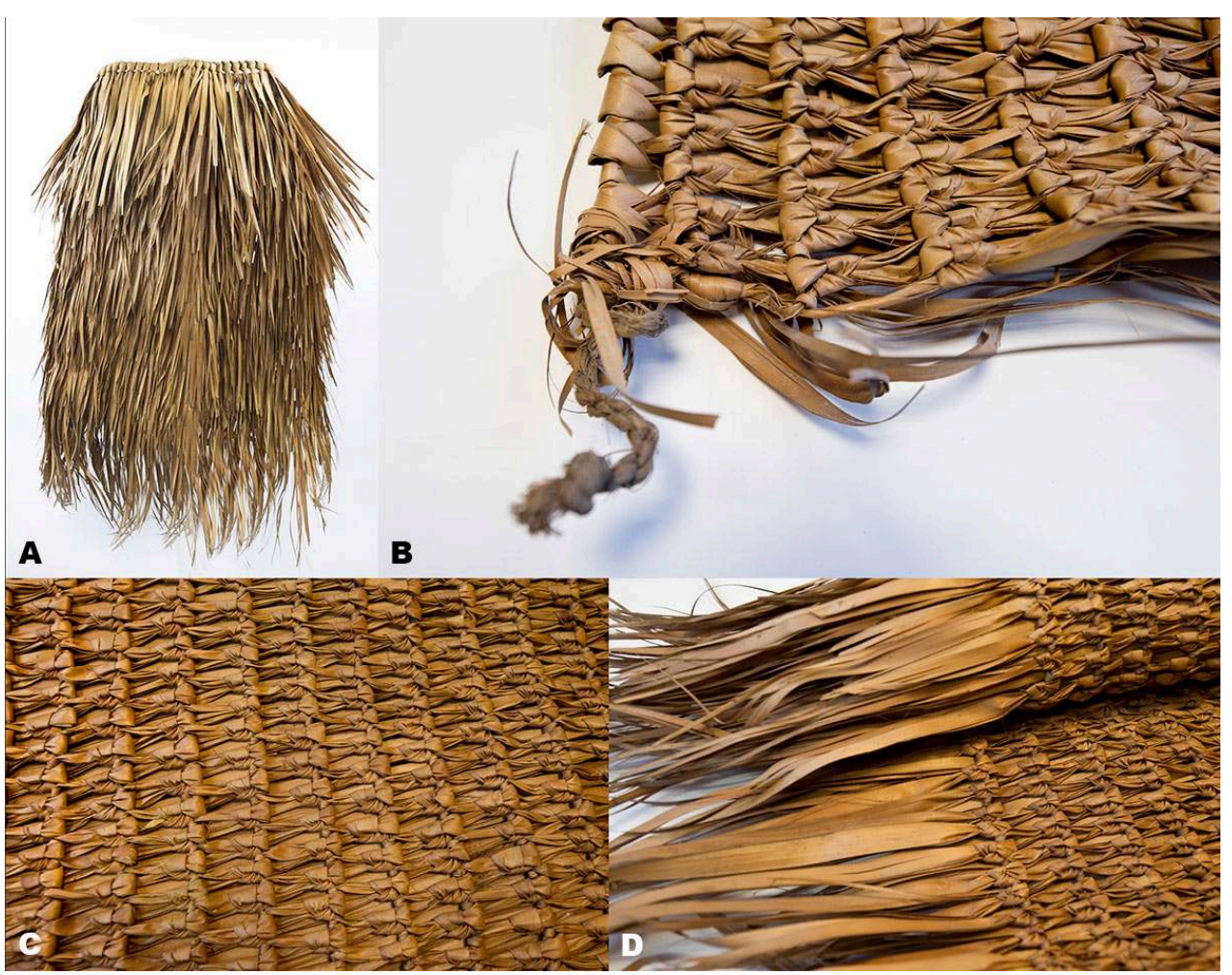

Détails du manteau MNHN-E-2002.1.37 : B. l'accroche supérieure, C. l'aspect de l'intérieur et D. la frange inférieure. Ce nœud (C) est identique à celui des capes $\S \S 13$ et 14, il est simplement inversé : on voit à l'intérieur la boucle qui est à l'extérieur dans les deux autres manteaux.

(c) N. Idalie

64 - Nahua. Pahuatlán (Puebla) ${ }^{6}$

- En palme ; Sierra Norte de Puebla

- Date inconnue (vers 1955) ; collecteur Guy Stresser-Péan

- Longueur $102 \mathrm{~cm}$

- MNHN-E-2002.1.37

\section{Sacs et musettes (morrales)}

65 Devant travailler plusieurs heures dans sa parcelle, le paysan emporte avec lui, outre de quoi se désaltérer (voir ci-dessus $\S \S 6-8$ ), dans des sacs, besaces ou musettes ce qu'il faut pour improviser un feu de bois où réchauffer les tortillas préparées le matin (Figure 51). Lieu de vie et de partage, on peut même y dormir en cas d'intempérie ou de longues journées de travail, et lorsque cela est possible, s'y rassembler pour les fêtes.

66 Ces morrales peuvent aussi servir de contenant pour les grains de maïs et de haricots lors des semis. 
Figure 51 : « La comida de la parcela » (9/2017, Chiquilistlán, Jalisco)

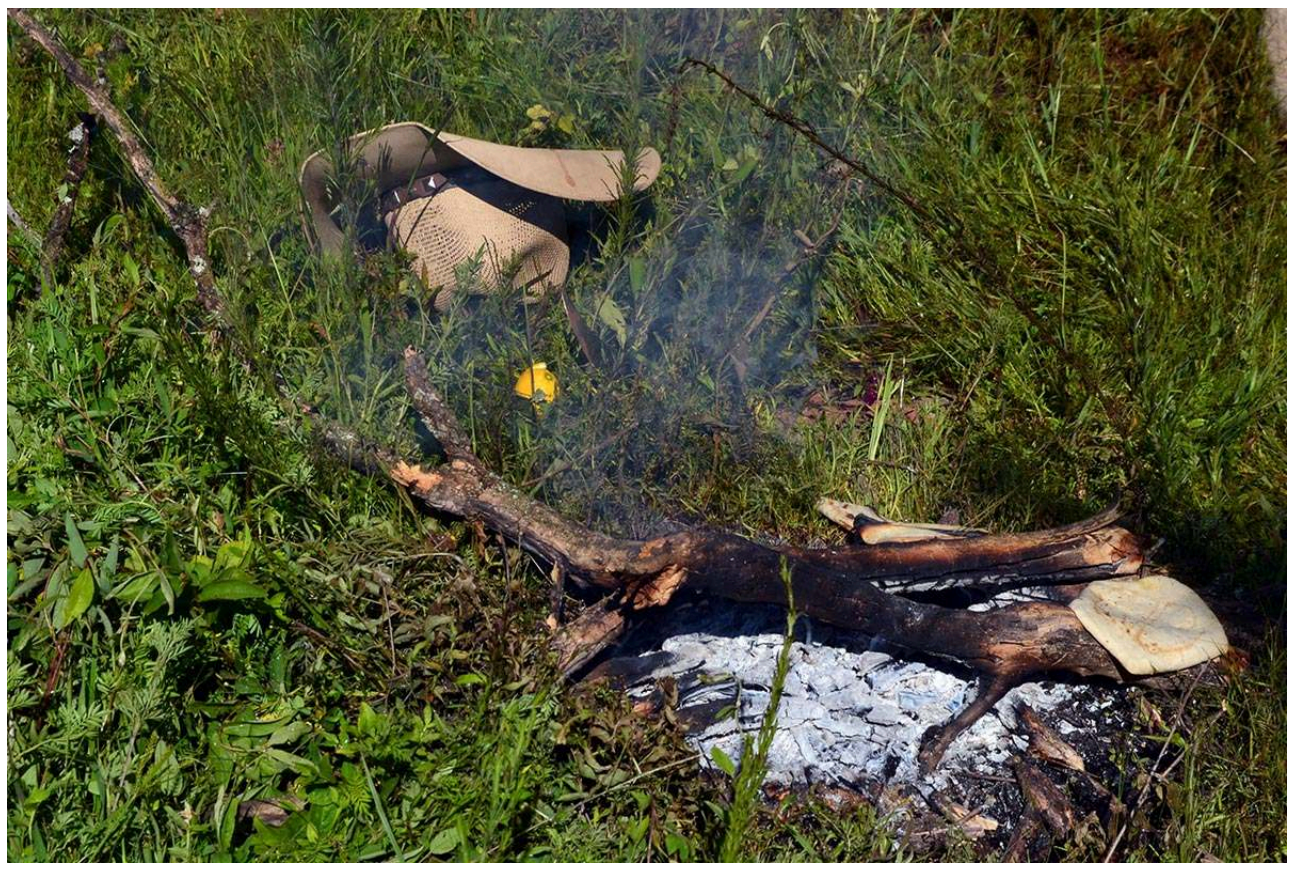

(C) P. Rameau

67 16) Boîtes d'allumettes

Figure 52 : Boîtes d'allumettes ETB-MX-RGA-2018-022 et 023
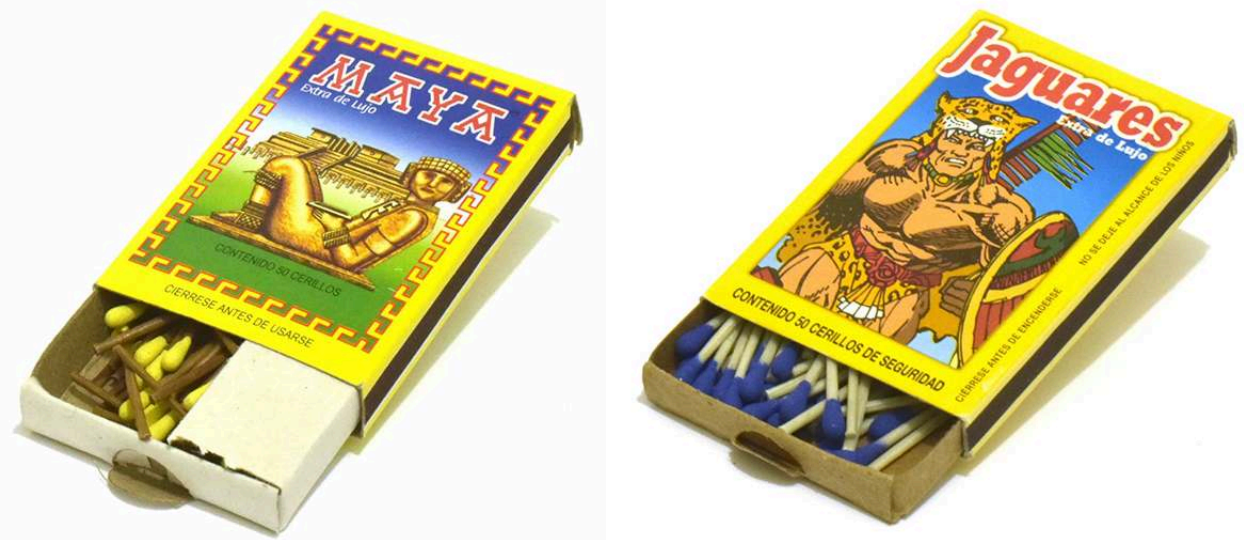

À gauche, allumettes MAYA (ETB-MX-RGA-2018-022), à droite Jaguares (ETB-MX-RGA-2018-023), Ville de Mexico, 7 × 4 × $1 \mathrm{~cm}$, collecteur Ruben García Andrade

(c) P. Rameau

68 17) Musettes, sacs à nourriture tissés 
Figure 53 : Musettes ETB-MX-SBPR-2017-080 et ETB-MX-PR-2016-007
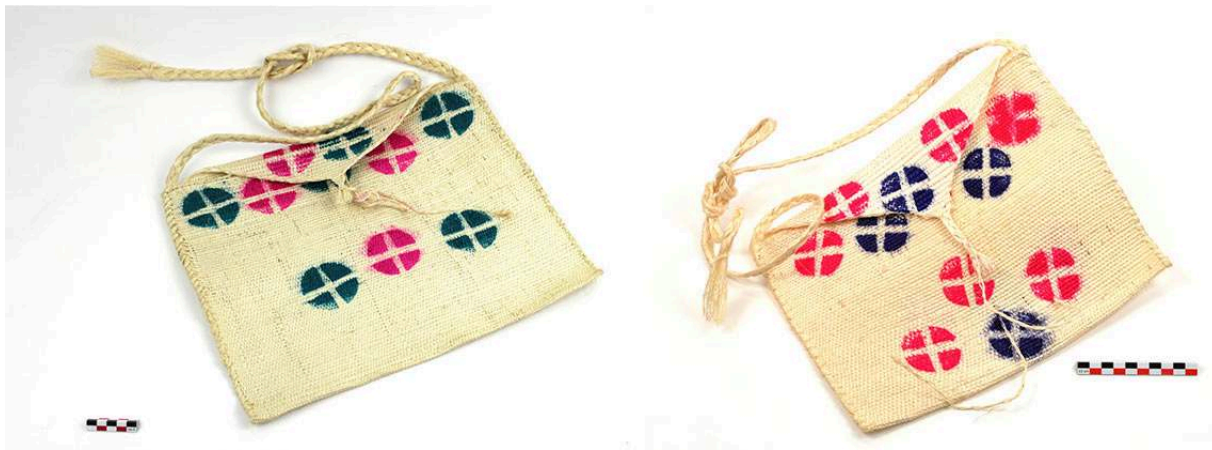

À gauche : Nahua. Cuetzatlan (Puebla) ; fibres d'agave tissées, 42 × 35 cm, 22/9/2017, ETB-MXSBPR-2017-080

À droite : bolsa de ixtle. Nahua. Sierra Norte de Puebla ; fibres d'agave tissées, $32 \times 25 \mathrm{~cm}, 2016$, don d'Alvaro Salgado, ETB-MX-PR-2016-007

(c) S. Bahuchet et P. Rameau

Le motif est imprimé avec un tronçon de bois fendu en quatre. Bien que collectés en des endroits différents, ces deux sacs similaires semblent provenir du même atelier. La bandoulière est une tresse de fibres d'agaves, qui est cousue à l'intérieur sur une dizaine de centimètres.

18) Sac à nourriture (morral)

Figure 54 : Sac à nourriture ETB-MX-SBPR-2017-202

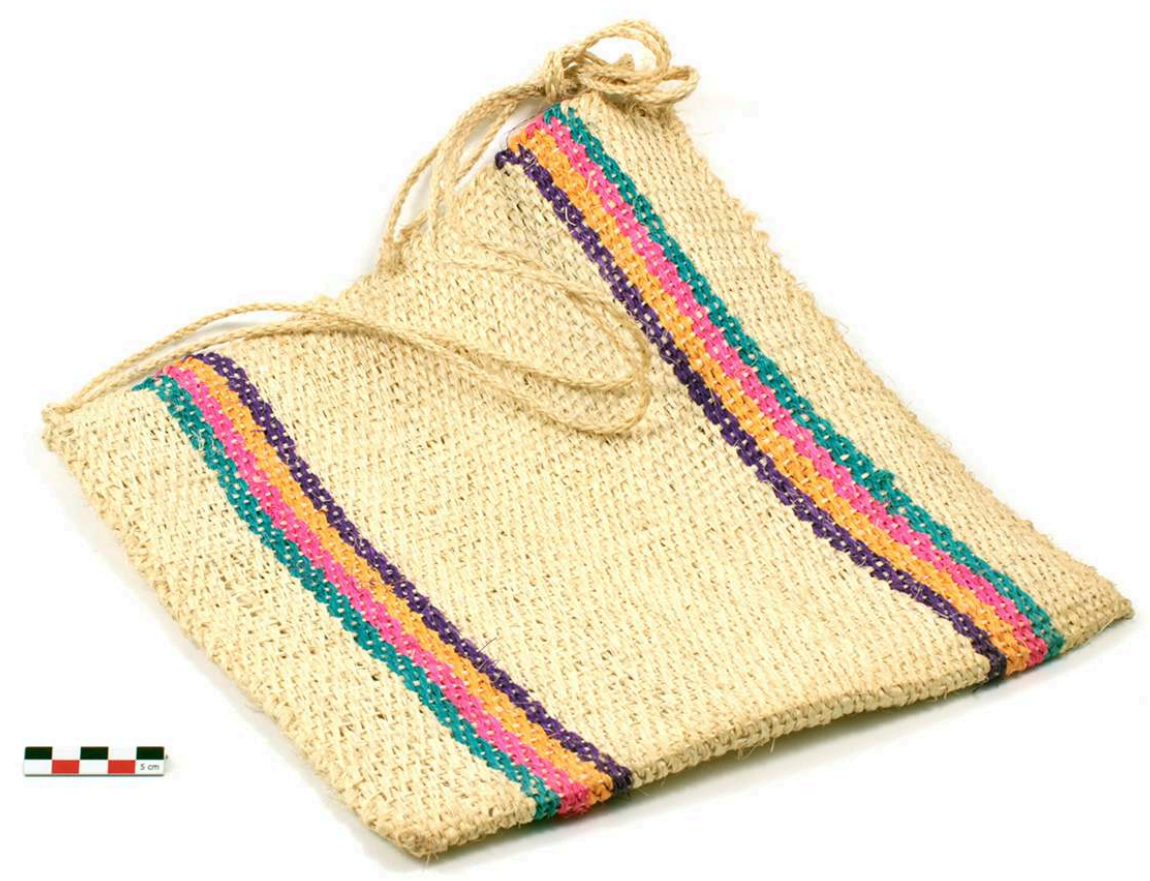

(c) P. Rameau

71 - Nahua. Cuetzatlan (Puebla), quincaillerie

- Fibre d'agave yute, tissée 
-22/9/2017 ; collecteurs Pauline Rameau et Serge Bahuchet

- $43 \times 37 \mathrm{~cm}$

- ETB-MX-SBPR-2017-202

19) Filets à nourriture (redes)

Figure 55 : Filets à nourriture ETB-MX-PR-2014-200 et 201

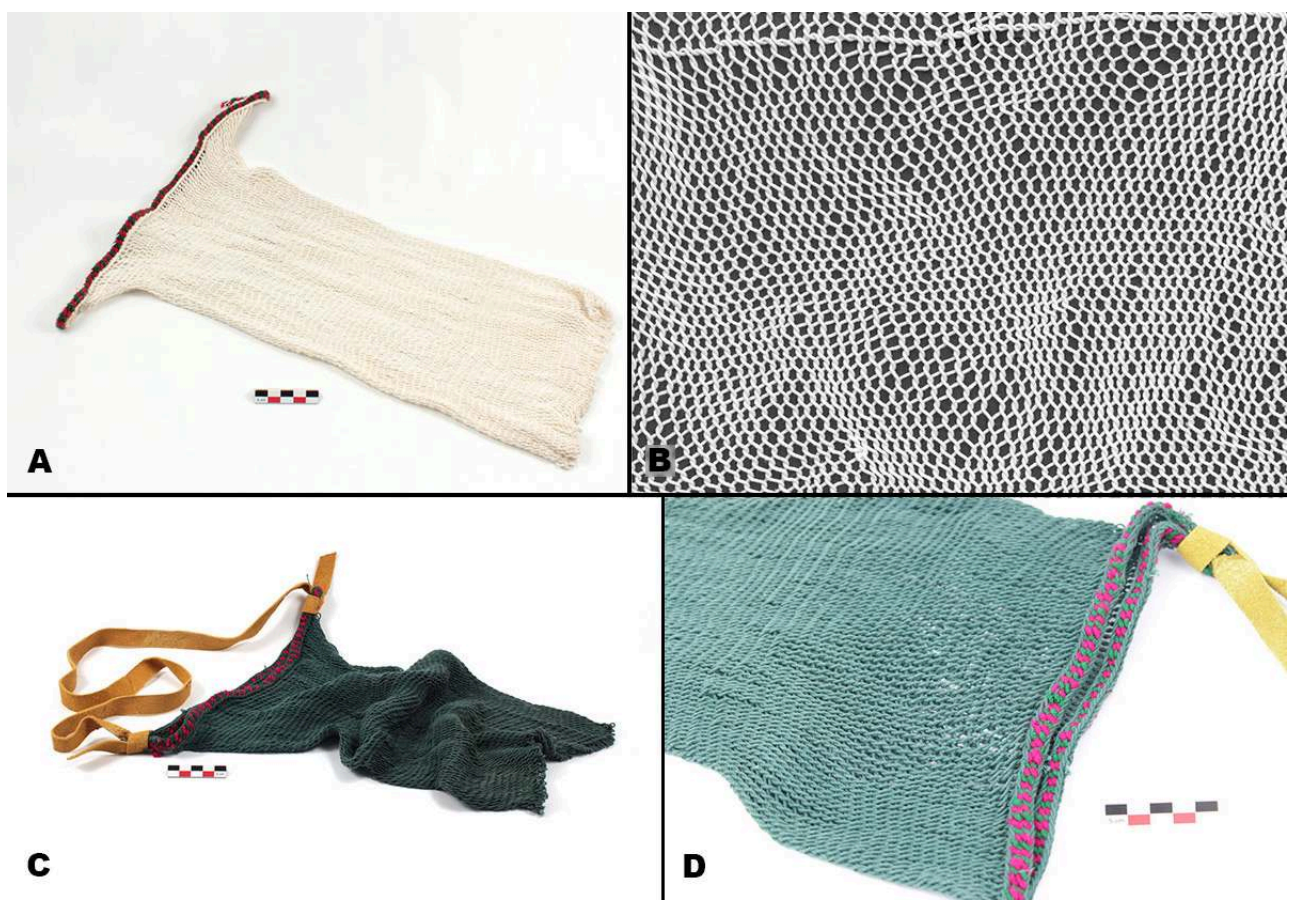

A, B : ETB-MX-PR-2014-200, et détail des mailles au fond du filet

C, D : ETB-MX-PR-2014-201, et détail des mailles et de l'attache. En haut, a et b : ETB-MX-

PR-2014-200, San Cristobal (Chiapas), fil de coton de Merida blanc, tressé ; 28 × 42 cm, 13/12/2014 En bas, c et d : ETB-MX-PR-2014-201, San Cristobal de las Casas (Chiapas), fil de coton teint avec sangle de cuir ; 41 × 20 cm, 13/12/2014, collectrice Pauline Rameau

(c) P. Rameau et S. Bahuchet

Ces deux filets sont extensibles. C'est une sorte de tressage en tube, avec une maille en boucle (nommée Linked hourglass looping en anglais, cf. Seiler-Baldinger 1994: 15-16).

74 20) Filet à nourriture (red)

Figure 56 -Filet à nourriture ETB-MX-PR-2014-134 et détail des mailles et de la boucle
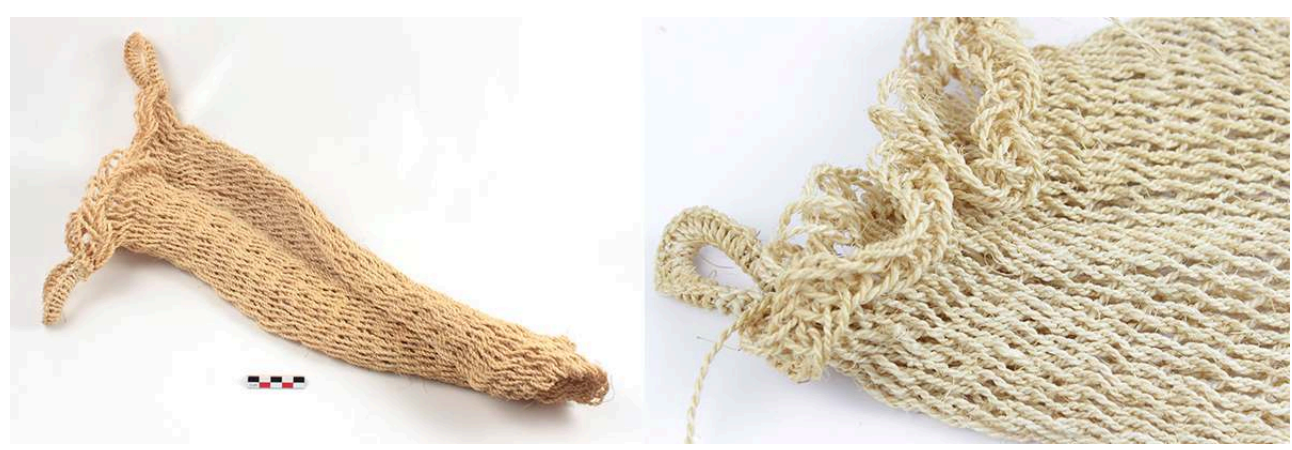

(c) P. Rameau et S. Bahuchet 
- San Pedro Cajonos (Oaxaca), au marché

- Corde de fibres d'agave ixtle, torsadée et tressée, avec deux anneaux noués pour passer une bandoulière

-21/12/2014 ; collectrice Pauline Rameau

- $50 \times 30 \mathrm{~cm}$

- ETB-MX-PR-2014-134

Les mailles sont similaires à celles des filets de coton ( $\$ 19)$, mais beaucoup plus grandes, de telle sorte que le tressage de la corde a pu être réalisé à la main.

21) Besace, filet à nourriture avec bretelle à coulisse, morral

Figure 57 : Besace ETB-MX-PR-2014-206 et détail du fond
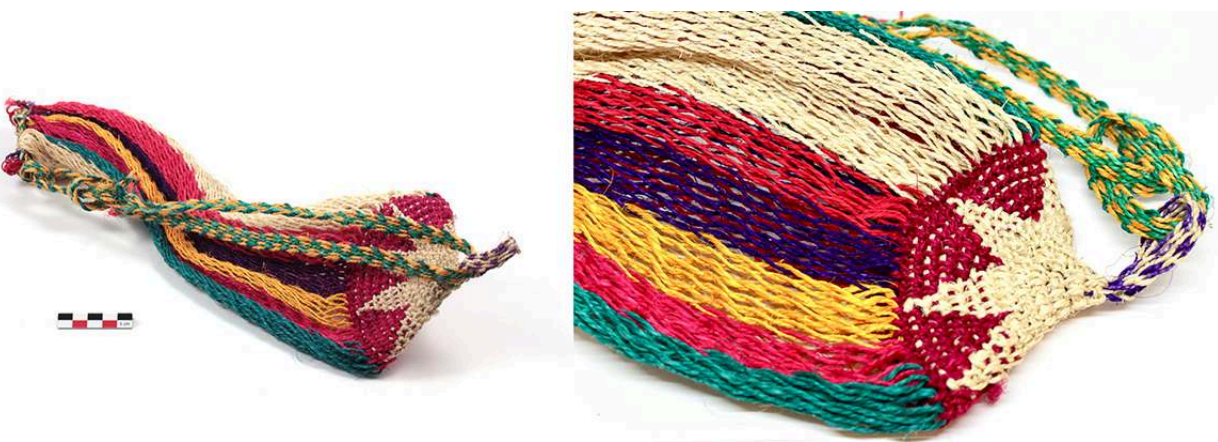

(C) P. Rameau et S. Bahuchet

- San Cristobal de las Casas (Chiapas)

- Corde de fibre d'agave ixtle, 5 anneaux de métal pour la coulisse. Le fond est conique, avec des nœuds en boucle fixés sur une corde en spirale. Les boucles du filet, en «sablier » (cf. § 19), partent sur le dernier rang du cône, qui d'ailleurs est le point de départ du tressage

-13/12/2014 ; collectrice Pauline Rameau

- $45 \times 25 \mathrm{~cm}$

• ETB-MX-PR-2014-206

\section{Le travail dans les champs}

En ce qui concerne l'agriculture et les outils associés, nous nous inspirons de Bonfil Batalla (2012). Présentant les outils, celui-ci distingue les étapes du travail du système agricole de la manière suivante (Bonfil 2012 : 54-55) :

80 - Préparation du terrain

- Nettoyage du terrain

- Labour

- Semailles

- Travail sur les cultures

- Récolte

- Transport

- Magasinage.

81 Dans les présentations qui vont suivre, nous regrouperons certaines de ses étapes. Certains outils sont à usages multiples et interviennent à diverses périodes, en 
particulier les outils coupants (machettes, serpes) qui servent à la préparation et au nettoyage. D'autres sont beaucoup plus spécifiques, les houes-bêches coas pour les semis et les couteaux-éplucheurs piscalones pour la récolte. Les contenants, paniers, hottes, sacs, sont aussi à usages multiples, bien qu'ils interviennent d'une manière inévitable pour certaines étapes du système agricole (récolte, transport).

\section{La préparation et le nettoyage du terrain}

\section{Désherbage}

82 Machettes et serpes, emmanchées ou non, sont les outils à tout faire pour gérer la végétation, et notamment pour nettoyer le terrain avant les semis.

22) Serpes (casangas)

Figure 58 : Serpes ETB-MX-PR-2018-009 et ETB-MX-SB-2010A-026
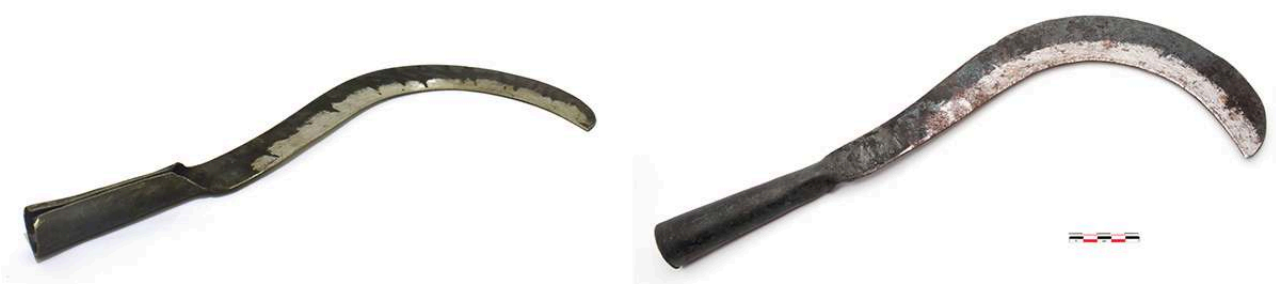

À gauche : ETB-MX-PR-2018-009, Sayula (Jalisco), L 46,5 cm, 20/4/2018

À droite : ETB-MX-SB-2010A-026, Cuautitlán (Etat de México), L 45 cm, 16/4/2010

(C) P. Rameau

Le fer peut être simplement tenu à la main ou bien être emmanché. La plupart des lames agricoles utilisées au Jalisco sont forgées à Sayula ou à Ayutla. 
Figure 59 : La serpe permet aussi des travaux minutieux, comme nettoyer une feuille de nopal (cactus) de ses épines (Don Lauro, Agua Prieta, Chiquilistlán, Jalisco, 8/7/2017)

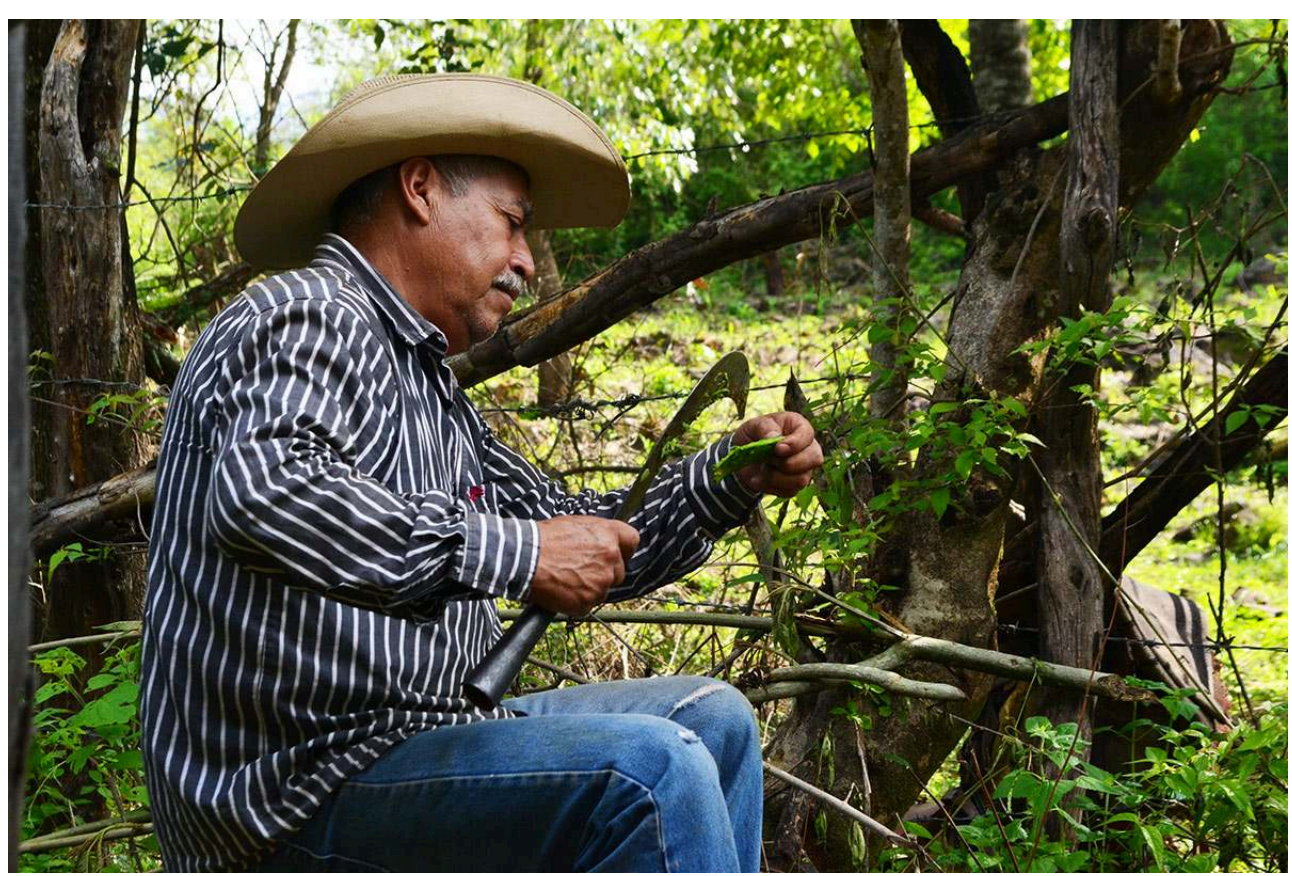

(c) P. Rameau

23) Serpe (guardaña)

Figure 60 : Serpe ETB-MX-FGTG-2021-002
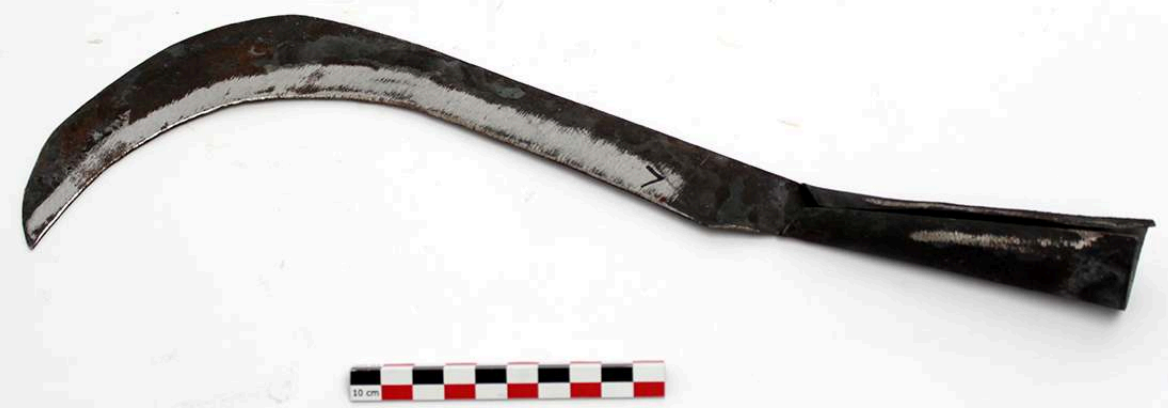

(c) S. Bahuchet

86 - P'urhépecha ; Cherán (Michoacán)

- Fer mince forgé ; à la fois pour désherber et pour couper les feuilles du maìs (cf. § 36)

- 21/6/2021 ; collecteurs Florencia Grisanti et Tito González García (Ritual Inhabitual)

-38x 13 x ø 3cm, épaisseur du métal $2 \mathrm{~mm}$

- ETB-MX-FGTG-2021-002 


\section{Préparation du sol et labour} houes. L'exemple qui nous a été rapporté est celui de Cherán dans la communauté p'urhépecha, où la houe azadón est utilisée pour labourer la terre et la préparer pour semer (Florencia Grisanti \& Tito González García, comm. pers. 21/6/21). Elisabeth Motte-Florac, dans son étude très complète sur le maïs chez les P'urhépecha de la Sierra Tarasca précise que la houe « est utilisée pour les travaux dans les solares et dans les terrains de superficie limitée », alors que le « bâton-plantoir » coa « continue à être parfois utilisé dans les terrains très pentus et de surface réduite. » (Motte-Florac 1988 : 61), l'outil usuel étant l'araire.

91 24) Fer de houe (azadón)

Figure 61 : Fer de houe ETB-MX-FGTG-2021-001

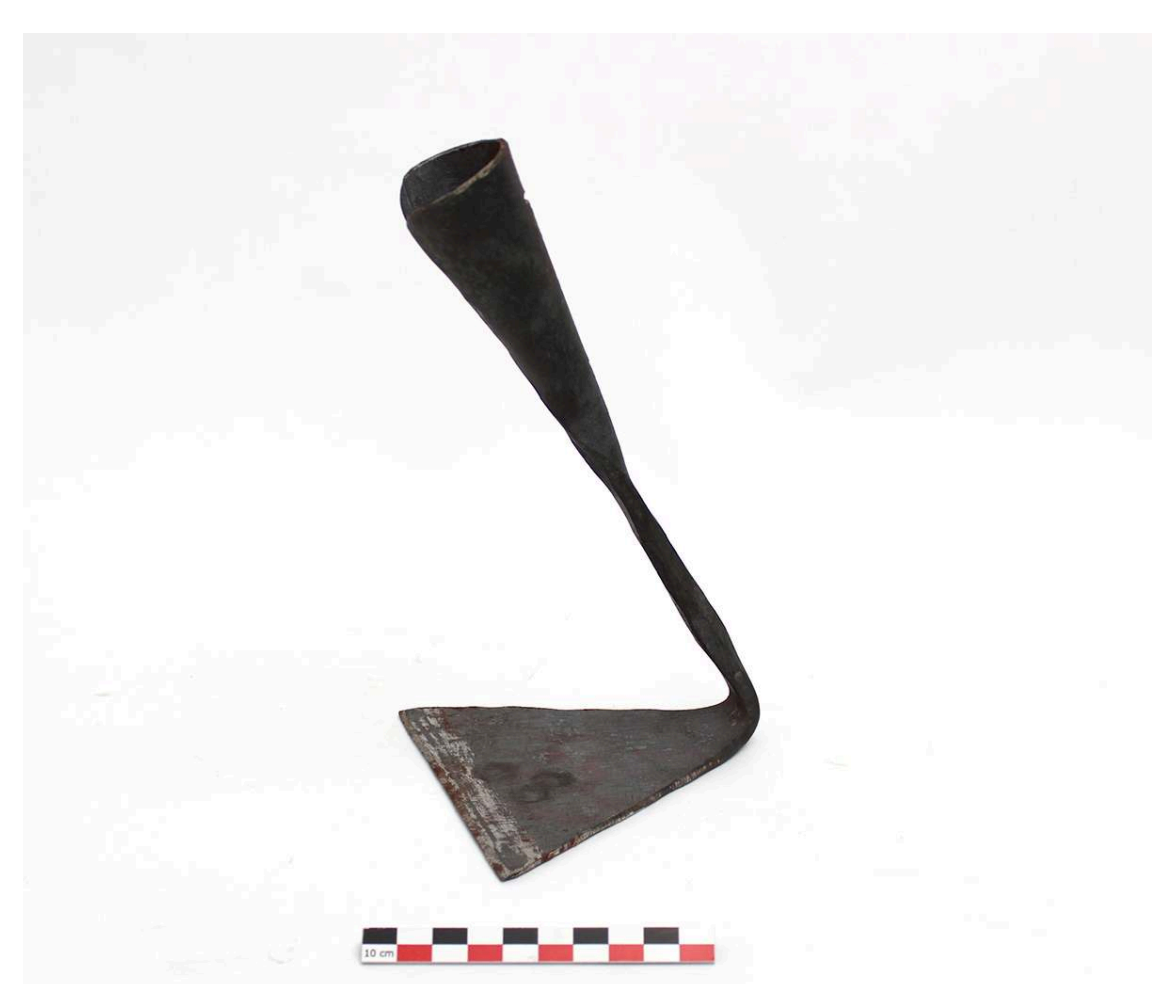


- P’urhépecha ; Cherán (Michoacán)

- Fer forgé ; à douille fermée, s'enfile à l'extrémité d'un manche de bois et se fixe avec un clou à travers une fente ; pour labourer la terre et la préparer pour semer dans les jardins solares, et pour les désherber (Figure 62)

-21/6/2021 ; collecteurs Florencia Grisanti et Tito González García (Ritual Inhabitual)

- $20 \times 11 \times 11 \mathrm{~cm}$

- ETB-MX-FGTG-2021-001

Figure 62 - Désherbage et buttage à la houe des plants de maïs dans le jardin (Cherán, Michoacán, juillet 2021)

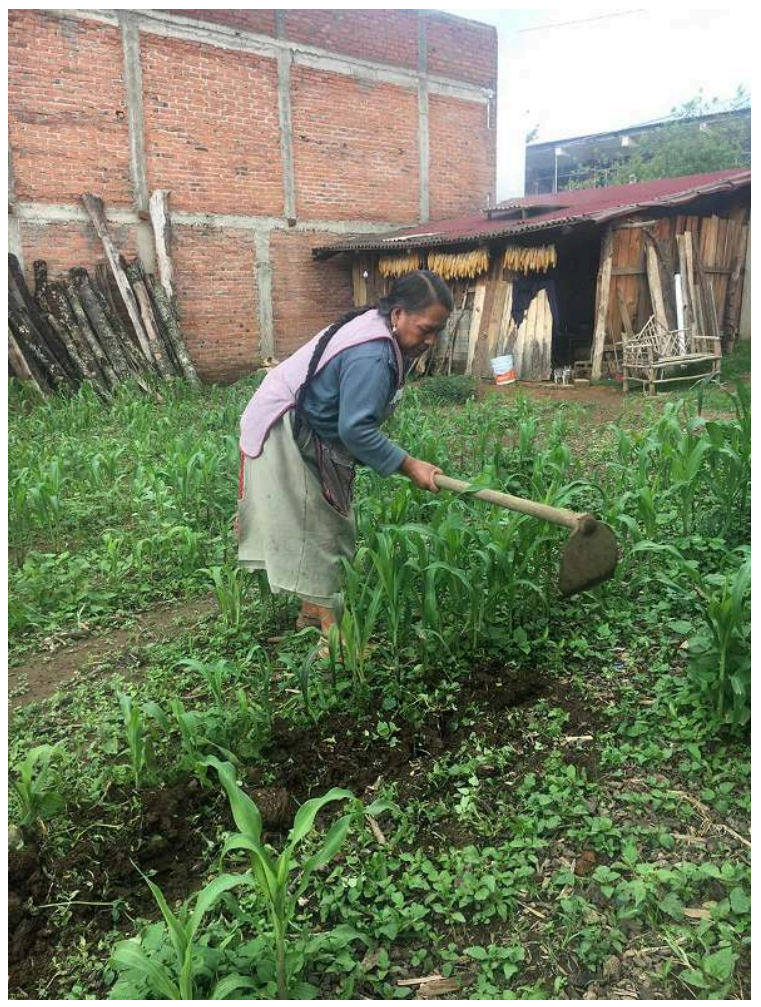

On remarque les épis de maïs suspendus sous le toit de la grange.

(c) Ritual Inhabitual

25) Houe emmanchée (coa) 


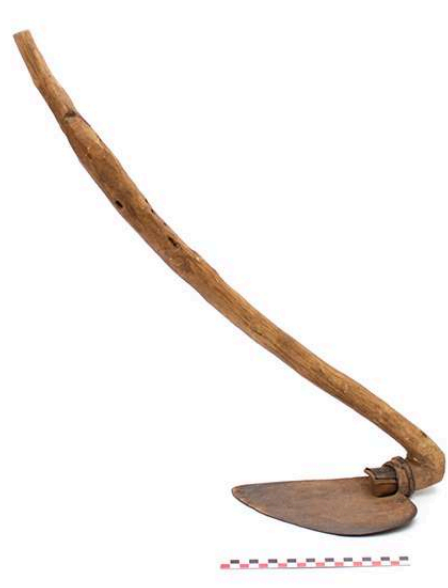

(c) S. Bahuchet

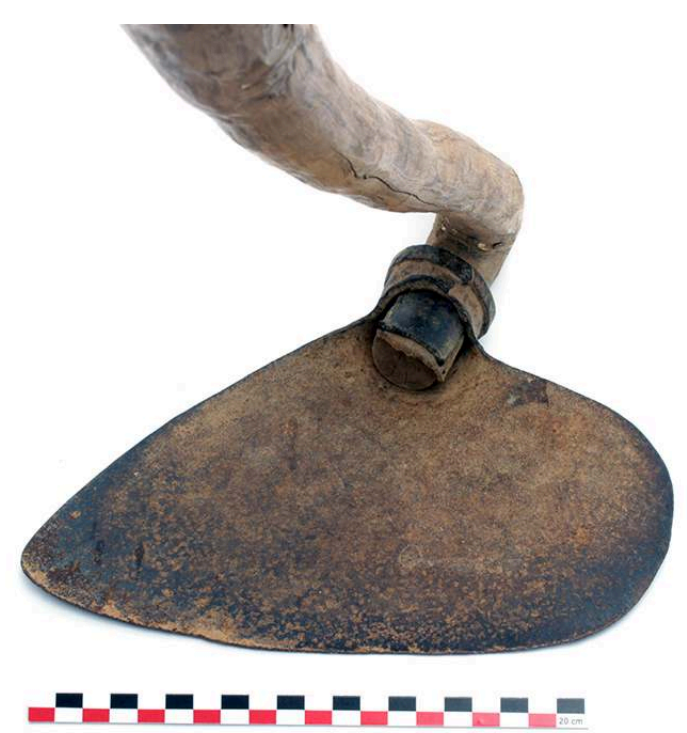

- Talea de Castro, Sierra Norte de Oaxaca (Oaxaca); achat dans la rue

- Fer, bois ; fixation à collet; le manche est coudé mais la lame, perpendiculaire au manche, est asymétrique, ressemblant ainsi à certaines coas (cf. § 27)

-18/11/2014 ; collectrice Pauline Rameau

- Longueur du manche $75 \mathrm{~cm}$; fer 25,5 x $16 \mathrm{~cm}$

- ETB-MX-PR-2014-107

\section{Araire et charrue}

C'est la forme qui distingue les deux instruments : l'araire est fait d'un soc placé au bout d'un axe rectiligne, le dental (ou sep); c'est un appareil symétrique qui gratte ou ouvre le sol, en surface et rejette, sur les deux côtés de la raie, la terre déplacée et émiettée par le soc (Figures 64-66).

Inversement, la charrue est asymétrique. Son soc n'est pas symétrique et la bande de terre qu'il découpe horizontalement est plus large d'un côté de l'appareil que de l'autre. La terre ainsi soulevée est rejetée et versée de ce même côté par une large lame axillaire, le versoir (Figures 67, 68) ; cf. les définitions dans Haudricourt \& Jean-Bruhnes Delamarre 1955 : 14-17).

Les deux instruments, araire et charrue, sont originaires d'Europe et ont été introduits lors de la colonisation espagnole. À l'évidence, l'araire a été apporté par les premiers colons, la charrue ultérieurement. Les communautés contemporaines qui utilisent l'araire témoignent donc d'une pratique plus ancienne de la culture attelée. 
Figure 64 : Un araire en vente à la quincaillerie (Guadalajara, Jalisco, 2/4/2012)

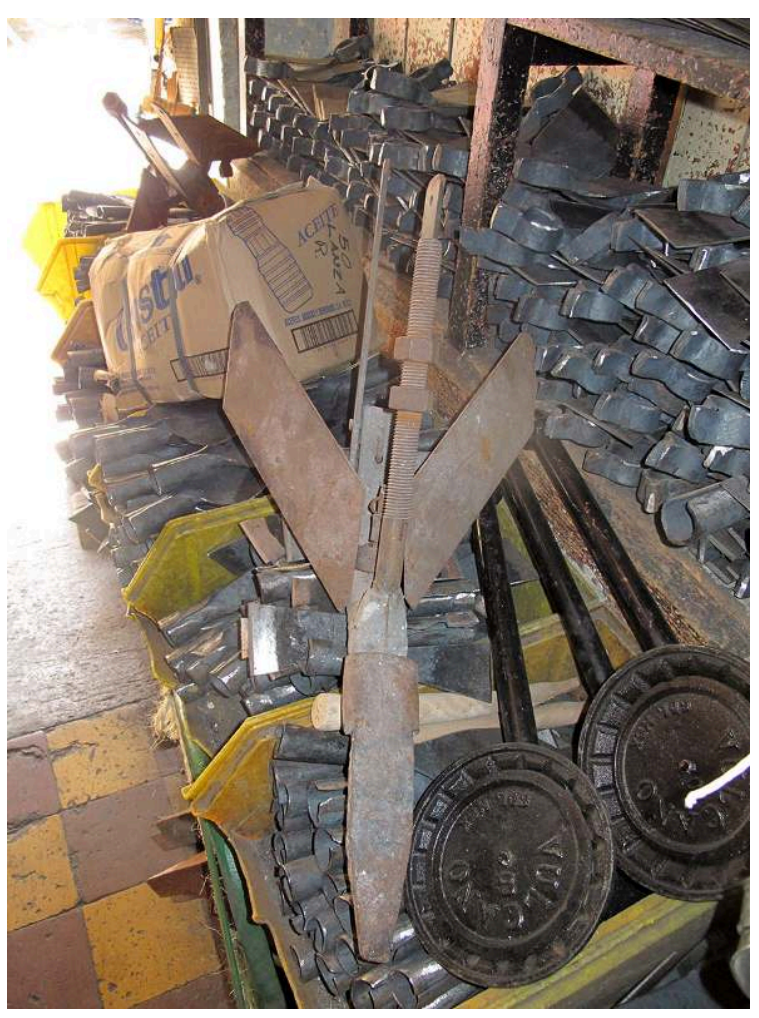

Cet araire a été soudé sur place. On en distingue clairement le soc et dans ce cas deux ailes latérales y ont été fixées, toujours symétriquement.

(c) S. Bahuchet 
Figure 65 : Araire (Talea de Castro, Sierra Norte de Oaxaca, 18/11/2014)

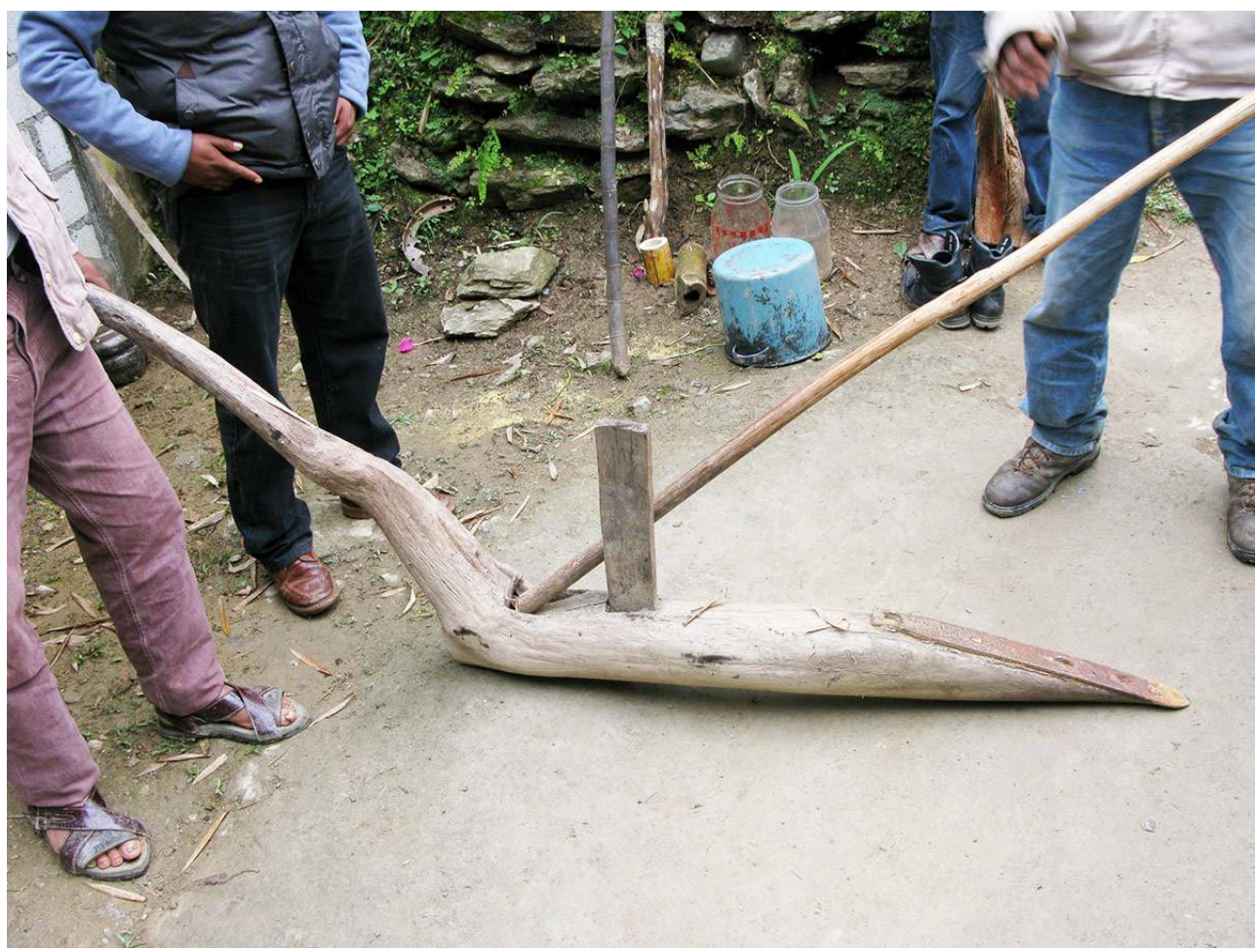

II s'agit d'un araire manche-sep, dont le manche et le sep, ou dental, sont une seule pièce de bois, sur laquelle le soc et le timon sont fixés. Le timon est la pièce par où s'exerce la traction de l'instrument, qui est guidé par le mancheron.

(c) P. Rameau

26) Maquette d'araire arado

Figure 66 : Maquette d'araire ETB-MX-SBPR-2014-012

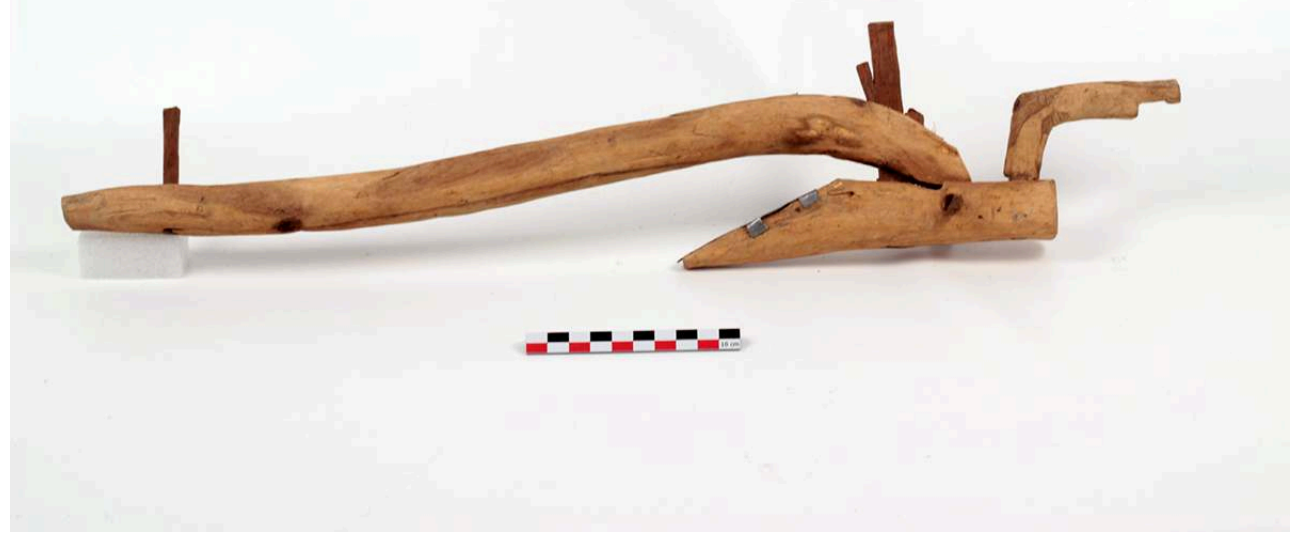

(C) P. Rameau 
99

Guadalajara (Jalisco), achat en brocante

- Bois, lame de métal pour le soc

-16/12/2014; collecteurs Pauline Rameau et Serge Bahuchet

-55,5 x $11 \times 3 \mathrm{~cm}$

- ETB-MX-SBPR-2014-012

100

Il s'agit d'un modèle "d'araire dental »: le mancheron unique est encastré dans le dental, à l'arrière du timon, lui-même également encastré dans le dental (Haudricourt \& Jean-Brunhes Delamare 1955 : 144)

101 27) Jouet-maquette de charrue

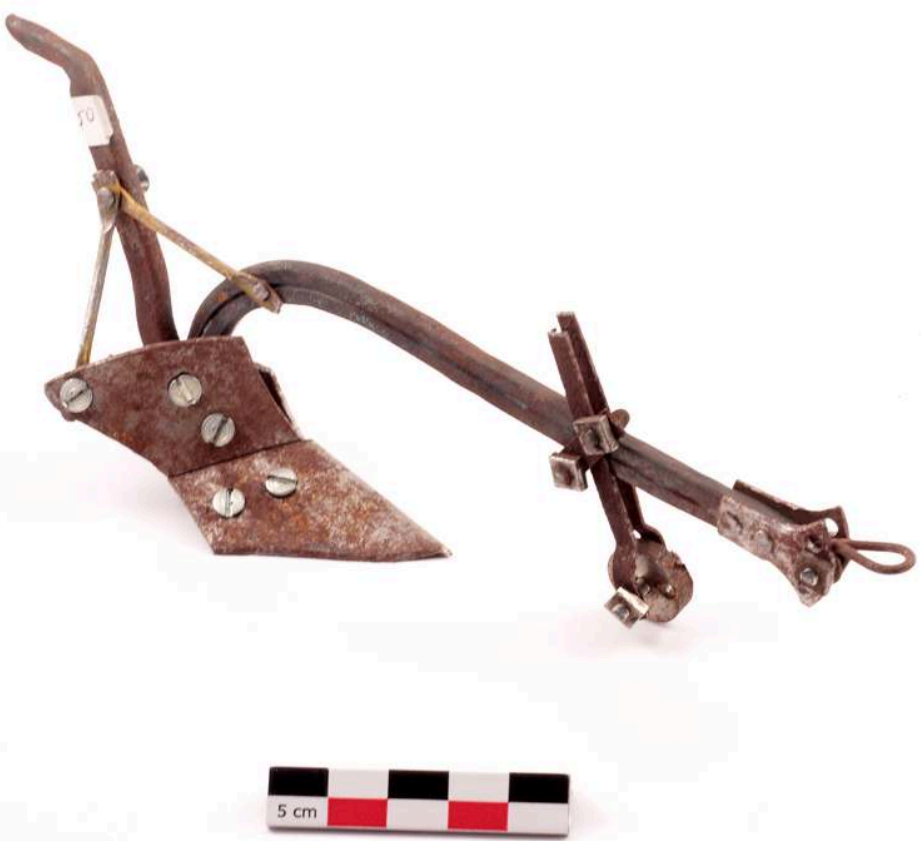

On distingue le versoir et la roue qui correspond à l'avant-train.

(c) P. Rameau

102 - Mascota (Jalisco)

- Fer forgé et soudé

-13/12/2014 ; collecteur Serge Bahuchet

$\cdot 20 \times 11 \times 3 \mathrm{~cm}$

- ETB-MX-SB-2014-071 
Figure 68 - Labour à la charrue dans la milpa de Don Camilo (Chiquilistlán, Jalisco, 11/7/2017) : en métal, sans avant-train

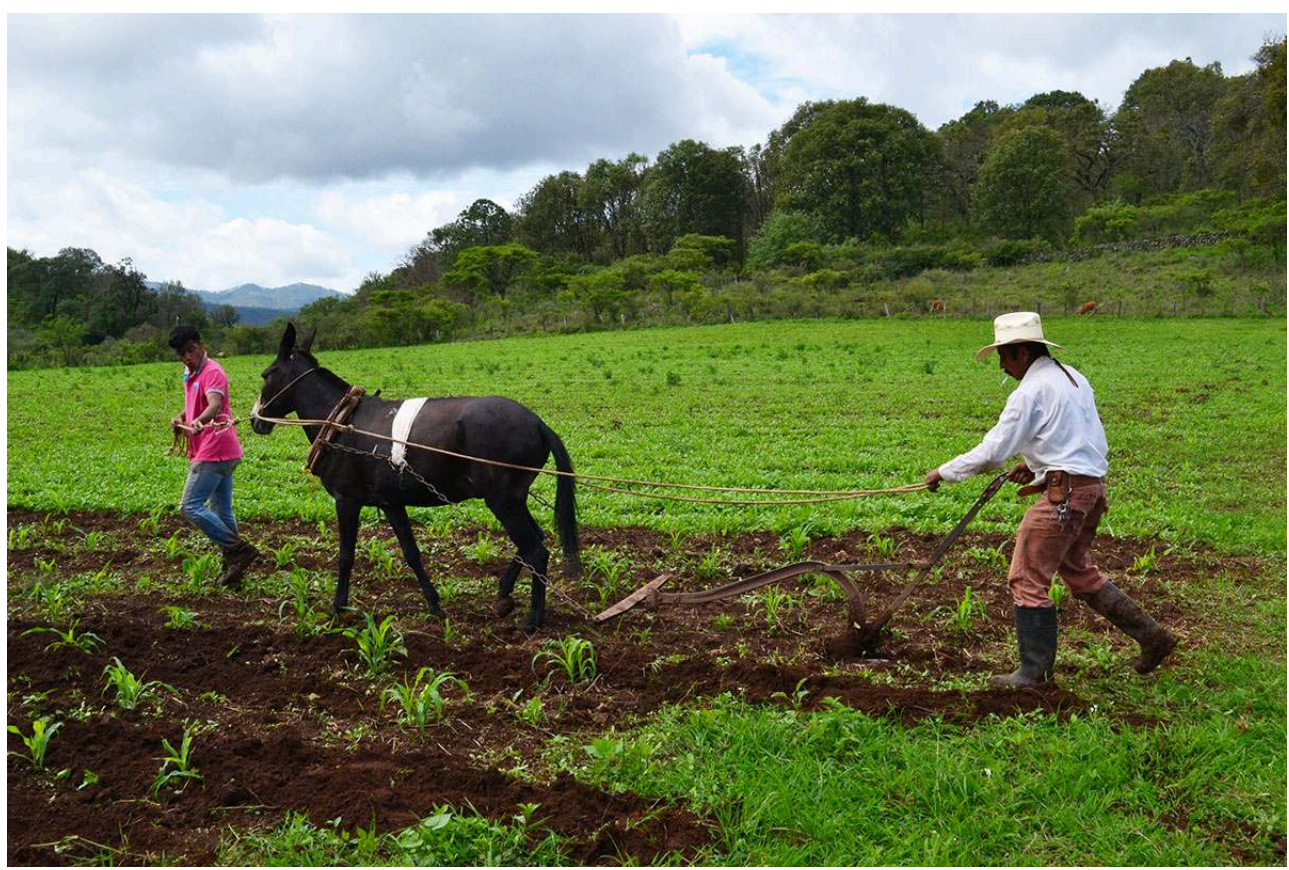

(c) P. Rameau

\section{Les semailles}

On trouve un joli petit texte sur les semailles dans l'Histoire naturelle de la Nouvelle Espagne de Francisco Hernández, écrit entre 1570 et 1577, mais publié en latin seulement en 1651 :

"On sème en mars, plaçant quatre ou cinq grains dans des trous espacés d'un pas, on ressème de nouveau en novembre, décembre et janvier, travaillant à nouveau la terre (...) on récolte plus tard ou plus tôt et on les garde [les grains] égrenés ${ }^{7} . »$ Chapitre XL sur le Maïs (Tlaolli) (traduit par nous de Barros \& Buenrostro 2007 : 92).

\section{Les coas, outils aratoires}

104 La coa est un outil agricole, proche d'une bêche, constitué d'une pointe métallique emmanchée. C'est un instrument original qui s'emploie en percussion lancée verticale ou légèrement oblique, punctiforme, le poids de la lame augmentant sa force. Il se situe entre le bâton à fouir avec une pointe renforcée, un plantoir, bien que le fer soit en général élargi et non une simple pointe, et une bêche mais il ne déplace pas la terre et l'on n'enfonce pas la lame en s'aidant d'un repose-pied (voir aussi Haudricourt 1987 : $115,120)$.

105 La coa est utilisée pour semer en saison des pluies dans les terres dures de montagne, aussi appelées coamiles, inaccessibles aux engins mécanisés. Toutefois cet outil peut aussi être utilisé en plaine pour former le trou où seront déposées les semences, recouvertes à l'aide du pied. Très souvent on loge dans le poquet entre 2 et 5 graines de maïs accompagnées d'une semence de haricot (Figure 69). 
Figure 69 : «Sembrando » : usage de la coa pour planter les semences, ici contenues dans une large bouteille de plastique. (21/6/2018, Chiquilistlán, Jalisco)

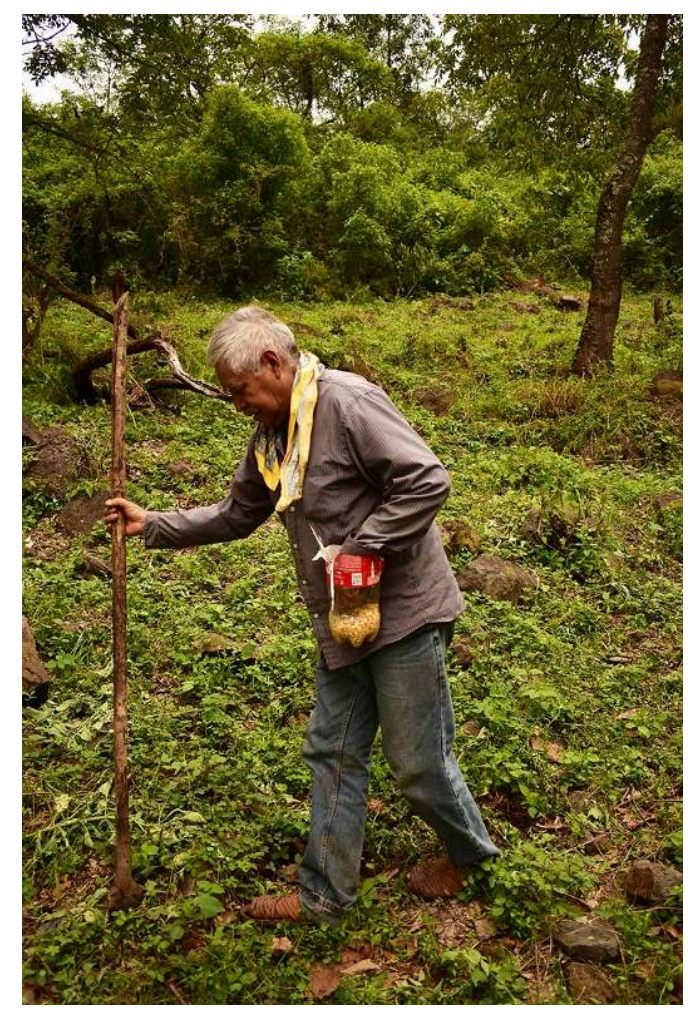

(c) P. Rameau

106 L'instrument est antérieur à l'arrivée des Espagnols. On en trouve deux représentations dans le Codex Florentin de Bernardo de Sahagun, au folio 72 du Quatrième livre. Ces images ne correspondent pas au texte qui les entoure, et de ce fait elles ne sont pas expliquées par une légende. La première, les semailles, montre un Aztèque tenant un bâton à fouir dans sa main gauche, et semant de sa main droite des grains dans le trou creusé ; il porte au cou un grand sac de toile que l'on pense contenir les semences (Figure 70). La deuxième image montre le cultivateur agenouillé creusant au pied des hautes tiges de maïs, probablement pour les désherber? La troisième illustre la récolte des épis par deux hommes. 


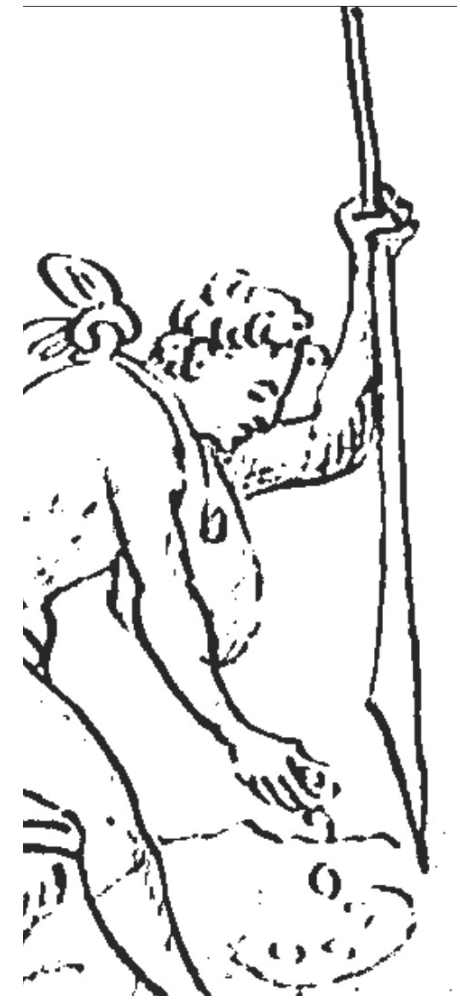

107 Sahagun emploie à plusieurs reprises le mot coa dans son texte, sans l'expliquer ni le mettre en contexte agricole (ce qui laisse à penser que ce terme était d'usage général à cette époque, compréhensible par tout Espagnol).

Ce mot coa ne semble pas avoir une origine bien définie. Il est bien présent dans l'espagnol du Mexique actuel, ainsi dans le Diccionario del español de México (qui reprend textuellement la définition du Diccionario del español usual en México de Luis Fernando Lara, 1996) :

Coa: s f Instrumento de labranza parecido al azadón, compuesto por un mango largo de madera unido a una pala de hierro terminada en punta, con uno de sus lados rectos y el otro curvo. ${ }^{8}$

Le Diccionario de Mexicanismos de Francisco Santamaría (2000) le définit ainsi :

Coa. Instrumento de labranza que sirve para cavar, como la pala. Consiste en une pieza, más o menos triangular, a modo de pala plana o ligeramente acanalada, de hiero grueso y con algùn filo en la línea de la base ; con mango largo y pesado, como asta, con el cual se maneja estando uno en pie. ${ }^{9}$

Le Diccionario de mejicanismos (Ramos I Duarte 1898) est plus ambigu, qui indique des régions particulières pour ce terme, avec des synonymes locaux :

Coa (Yucatan, Méjico, Michoacan), sf. : Instrumento de labor agrícola, a manera de hoz

Chuzo (Michoacan). sm., Coa: instrumento de labranza.

Tarécua (Hidalgo i Guerrero), sf. : Zapato viejo. Hay un instrumento agrícola con una punta corva i afilada por amboa lados, que en Yucatán llaman coa, i en Michoacán tarécua; término derivado del tarasco tarhekua, instrumento con que se cava ó labra la sementera, de tarheni, cavar ó labrar el campo. ${ }^{1011}$

Le Dictionnaire de la langue nahuatl ou mexicaine de Rémi Siméon (1885, consultable en ligne), donne le terme nahuatl : 
COAHUACATL : Bâton à fouir. 'huictli' ou 'coahuacatl', instrument qui servait pour le labourage. Il avait la forme d'un bâton dont l'une des extrémités était élargie et un peu coudée. $(. . .)^{12}$

\section{Avec l'explication suivante :}

«Coa, instrument aratoire fait d'une lame de métal sans rebord, rectiligne d'un côté, courbe de l'autre, et d'un manche en bois. L'instrument ancien était sans doute tout en bois ou avec une lame en pierre. La coa sert à faire des trous dans le sol dans lesquels sont enfoncés les graines. »

113 La description, répétée, d'une lame avec un côté rectiligne et un autre courbe, correspond peu ou prou au dessin du Codex. De nos jours, les formes des lames que l'on trouve dans diverses régions diffèrent. Certaines lames sont en effet asymétriques, en demi-cœur, avec la pointe dans l'axe du manche, le tranchant étant latéral ${ }^{13}$ ( $\$ 28$, Figure 72). D’autres sont lancéolées, pointues et symétrique, larges ou au contraire plus étroites ; les fers sont dans l'axe du manche (\$§ 29-32, Figures 71, 73-76).

Figure 71 : Deux coas lancéolées dans la grange (Jalpa, Chiquilistlán, Jalisco, 7/6/2017)

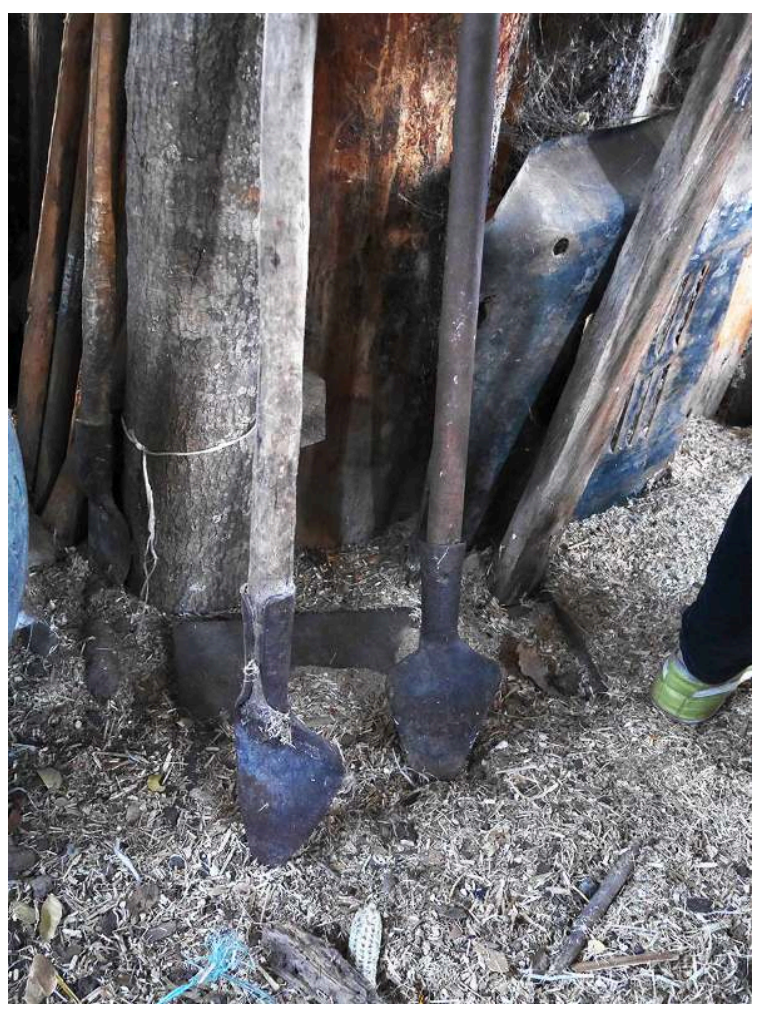

(c) S. Bahuchet

\section{Lame asymétrique}

28) Fer de bêche (coa) 
Figure 72 : Fer de coa asymétrique ETB-MX-SB-2010A-001

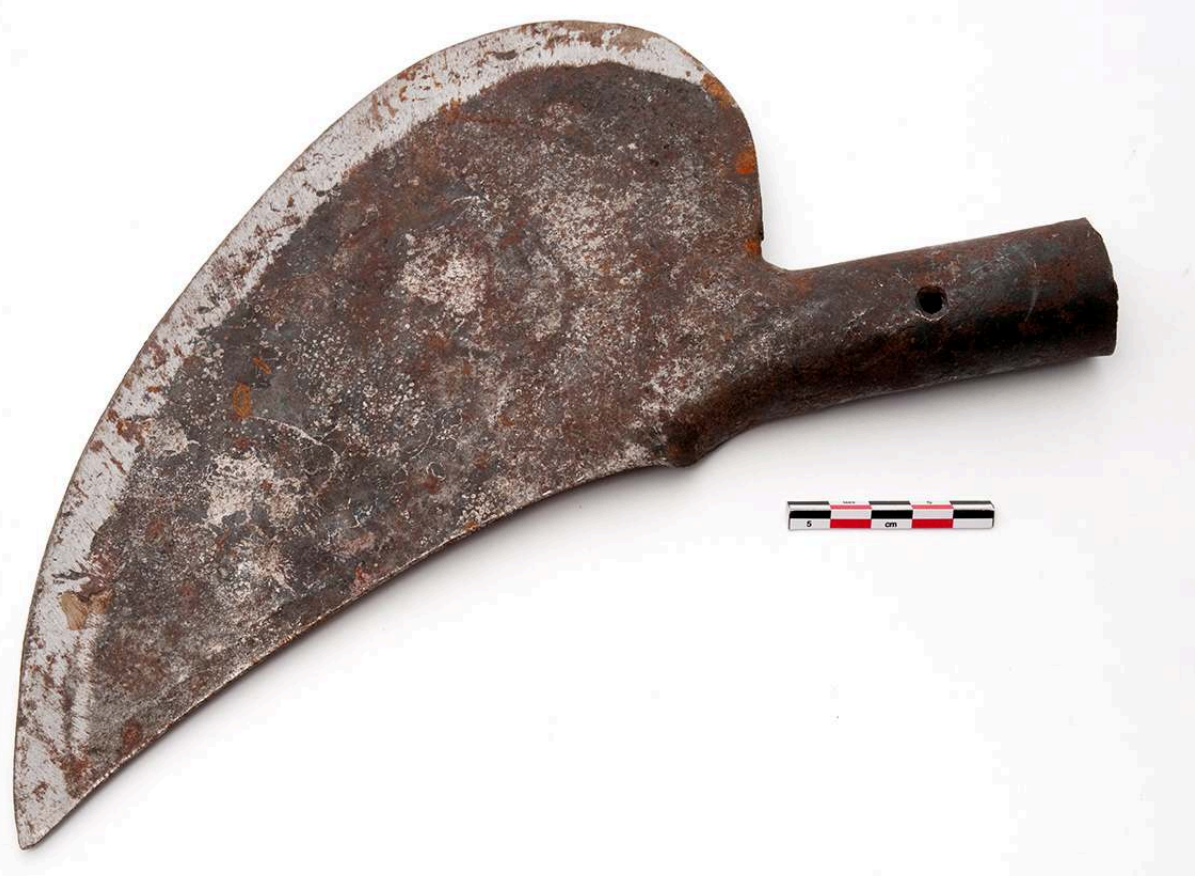

(c) P. Rameau

115 -Xochimilco (CDMX)

- Fer forgé devant être emmanché

- 5/4/2010 ; collecteur Serge Bahuchet

- $30 \times 14 \mathrm{~cm}$

- ETB-MX-SB-2010A-001

\section{Lame symétrique}

116 29) Bêches coa lancéolées

Figure 73 : Coas à lame symétrique ETB-MX-SB-2010A-029, ETB-MX-SB-2012-019 et ETB-MXSB-2014-013
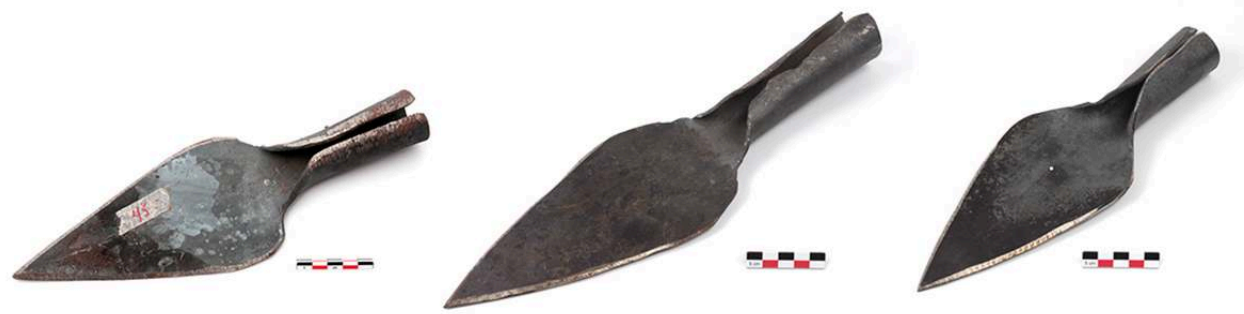

À gauche : ETB-MX-SB-2010A-029, Autlán (Jalisco), 29,5 x 10 cm, 20/4/2010

Au centre : ETB-MX-SB-2012-019, Guadalajara (Jalisco), 36 x $11 \mathrm{~cm}, 2 / 4 / 2012$

À droite : ETB-MX-SB-2014-013, Mascota (Jalisco), 28,5 x 10,5cm, 7/4/2014

(C) P. Rameau

117 30) Bêche coa emmanchée 
Figure 74 : Coa emmanchée ETB-MX-SBPR-2014-011

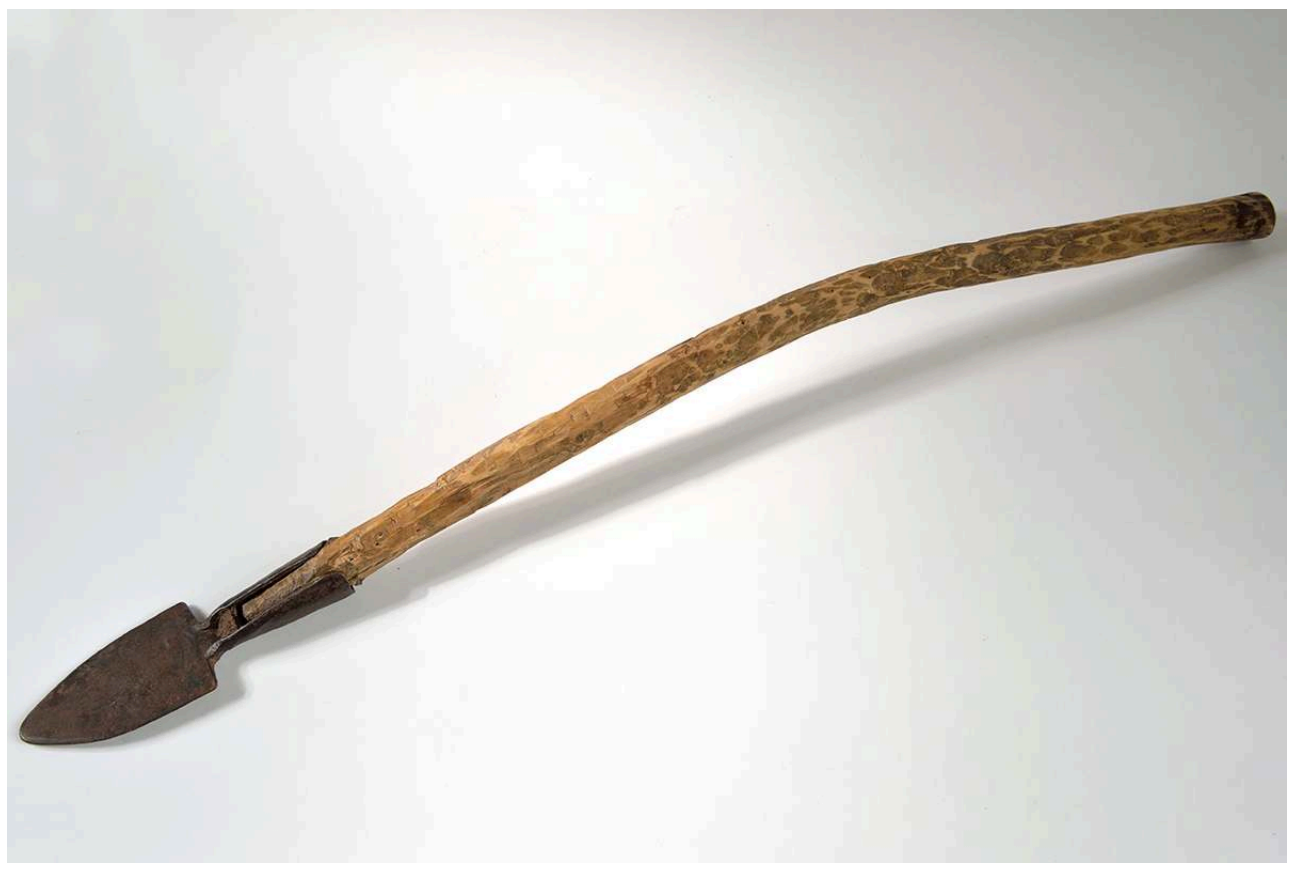

(c) J.-C. Domenech

118 - Guadalajara (Jalisco), achat en brocante.

- Bêche en métal, manche en bois

-16/12/2014 ; collecteurs Serge Bahuchet et Pauline Rameau

- ETB-MX-SBPR-2014-011 (Exposition permanente Galerie de l'Homme)

119 31) Lame de plantoir-bêche (coa sembradora)

Figure 75 : Lame de plantoir-bêche ETB-MX-PR-2014-211
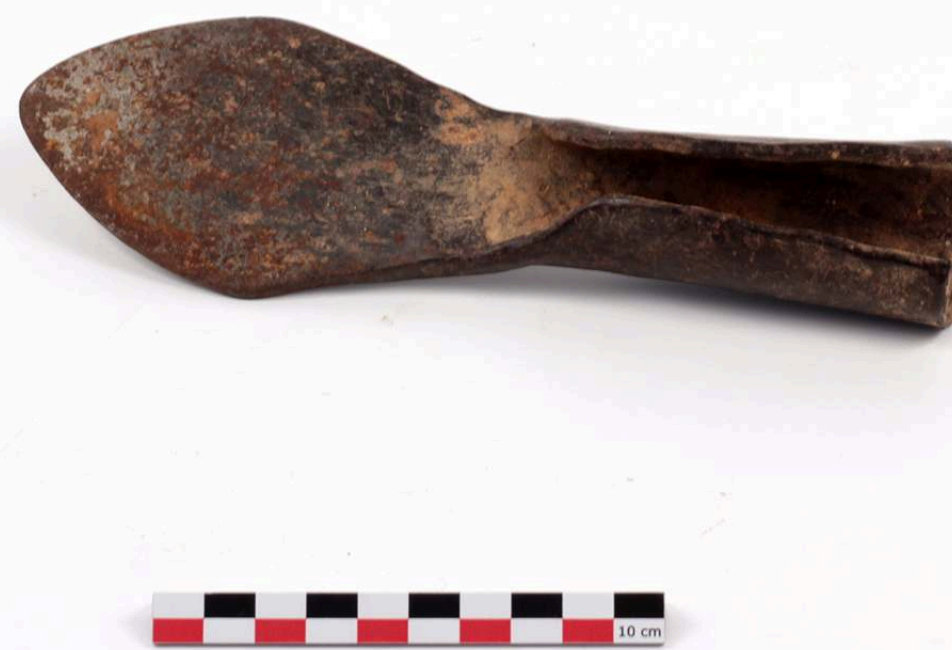

(c) P. Rameau

Revue d'ethnoécologie, Supplément 2 | 2021 
120 - Guadalajara (Jalisco), achat en brocante

- Fer. Ce petit fer, bien qu'épais, est plus un plantoir qu'un outil lourd capable de défoncer le sol.

-16/12/2014 ; collectrice Pauline Rameau

- $21 \times 7 \times ø 4 \mathrm{~cm}$

- ETB-MX-PR-2014-211

32) Lame de plantoir-bêche (chuzo)

Figure 76 : «Pic à semer » ETB-MX-PR-2014-068

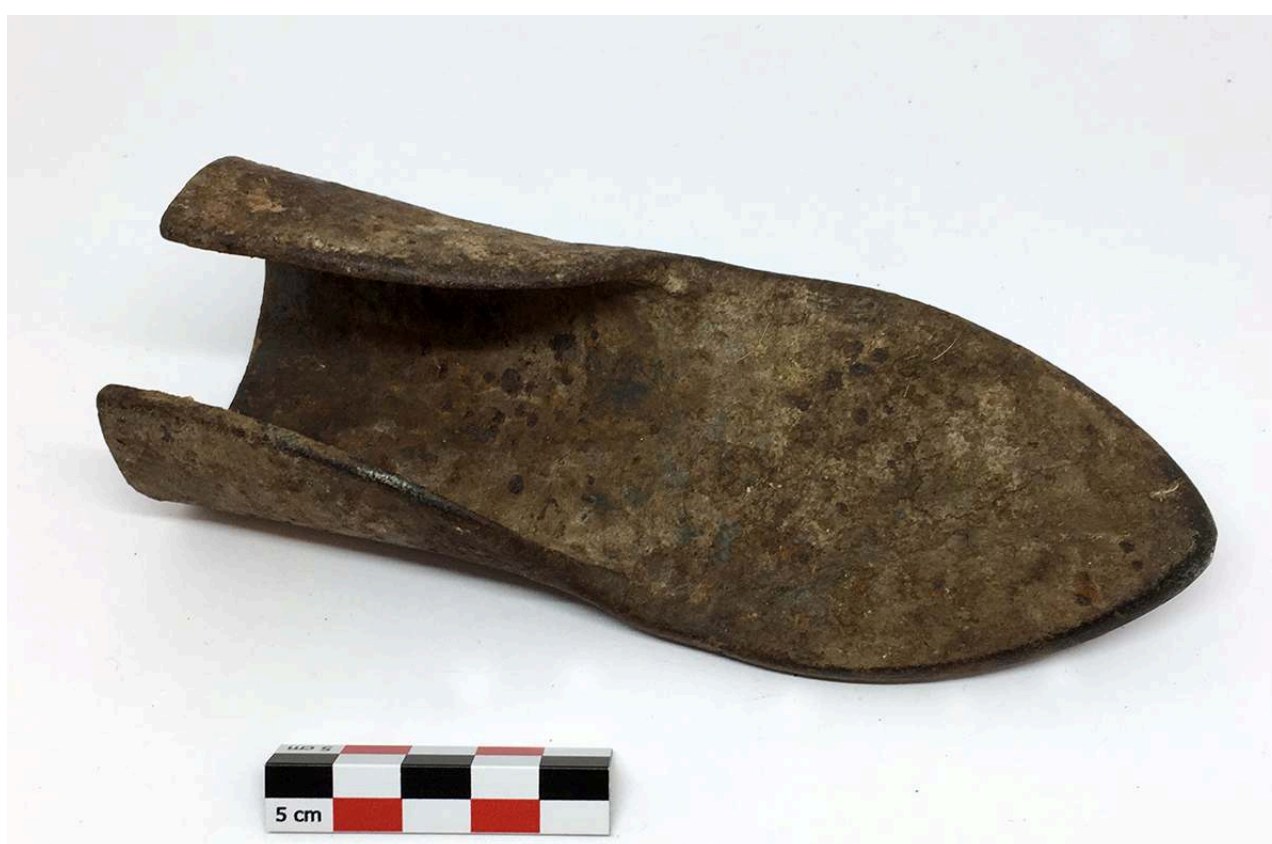

(c) S. Bahuchet

- Bienvenido, Tetela de Ocampo (Puebla)

- «Pic à semer » en fer. Ce petit fer épais et lourd est capable de défoncer le sol et en même temps de planter. Utilisé pour semer dans la montagne.

-11/11/2014, collectrice Pauline Rameau

$\cdot 16,5 \times 6,5 \times ø 5,5 \mathrm{~cm}$

- ETB-MX-PR-2014-068

33) Bêche droite (sembrador) 
Figure 77 - Plantoir-bêche sembrador ETB-MX-PR-2014-108

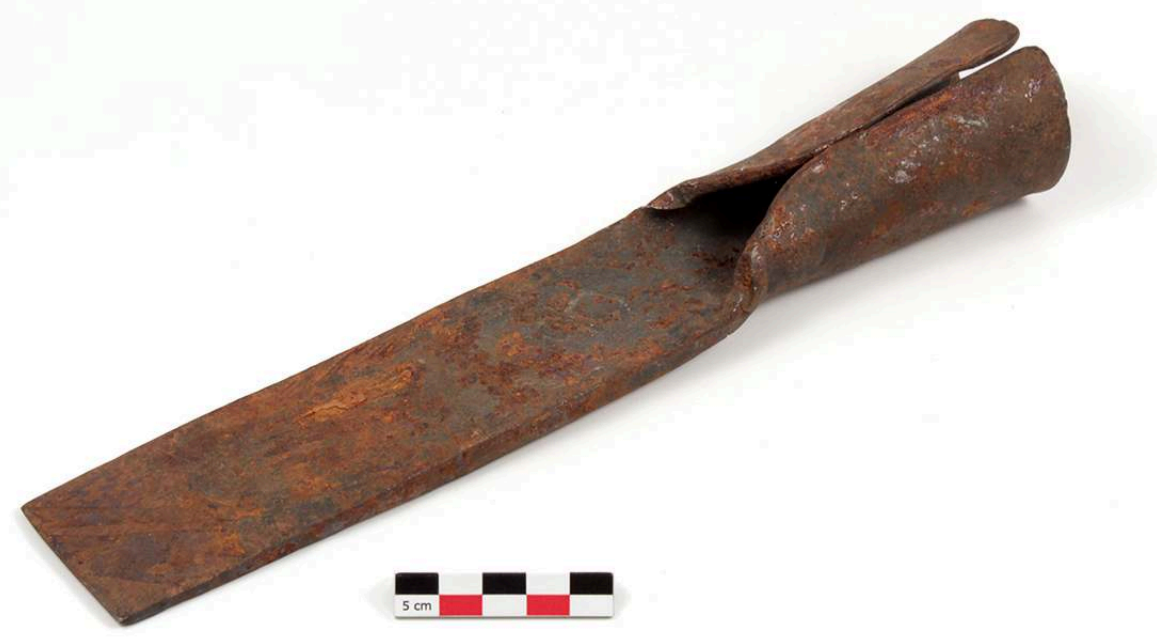

(c) P. Rameau

124 - Talea de Castro, Sierra Norte de Oaxaca (Oaxaca), achat dans la rue

- Acier provenant d'un ressort de voiture; cette lourde lame diffère des autres en n'étant pas lancéolée ; elle ressemble plus à un outil propre à défoncer le sol

-18/11/2014 ; collectrice Pauline Rameau

-28,5 X 4,6 X ø 4,5 cm

- ETB-MX-PR-2014-108

Plantoir

125 34) Pointe pour semer (pulson) 
Figure 78 : Pointe pour semer ETB-MX-PR-2014-176

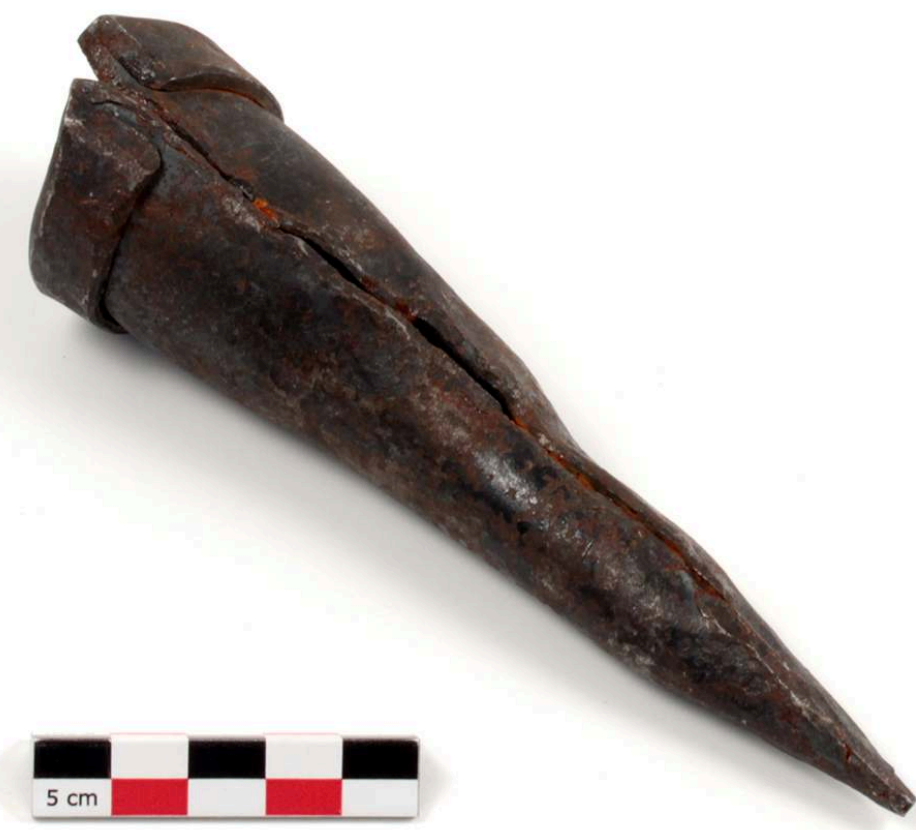

(c) P. Rameau

126 - Calle Comitan, San Cristobal de las Casas (Chiapas)

- Fer forgé, enroulé en cône ; se fixe à l'extrémité d'un manche droit

- 7/12/2014 ; collectrice Pauline Rameau

- L 15, $4 \mathrm{~cm}$

- ETB-MX-PR-2014-176 
Figure 79 : Semis avec le plantoir (Hidalgo, 27/6/2018)

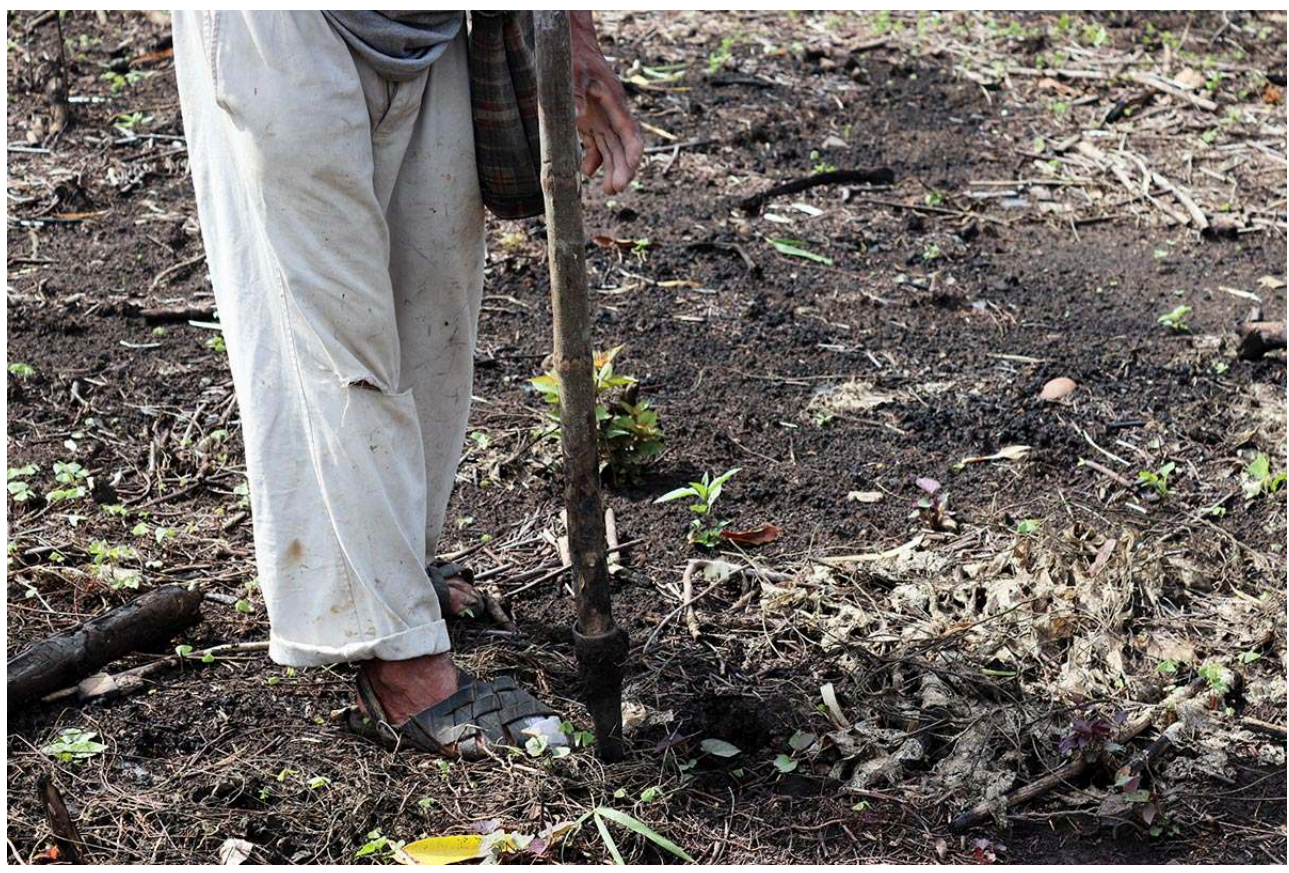

(C) P. Rameau

Dans certaines régions, en pays Nahua par exemple, la coa (devenue rare et remplacée par des bêches) sert à préparer le sol et à briser les mottes, alors que le plantoir sert spécifiquement pour les semailles (cf. Chamoux 1981 : 169-171).

\section{Semences et semailles}

\section{Sélection et reproduction des semences}

128 Dans l'attente des prochaines semailles, les semences sont précieusement gardées (voir $2^{\mathrm{e}}$ partie, $\S \S 61-64$, figures 124-126).

129 C'est à l'arrivée des pluies que l'on ensemence les champs. Les graines peuvent être soigneusement triées afin de conserver une ou plusieurs variétés en particulier, ou bien semées en mélange afin d'obtenir des variétés multicolores. Cette sélection, faite chaque année, est intimement liée aux conditions topographiques et climatiques bien sûr mais également aux pratiques socio-culturelles telles que les fêtes ainsi qu'aux préférences plus personnelles relatives aux saveurs, textures et couleurs recherchées.

130 35) Grand panier à semences, à couvercle (tenate) 
Figure 80 : Grand panier à semences ETB-MX-SB-2015-029

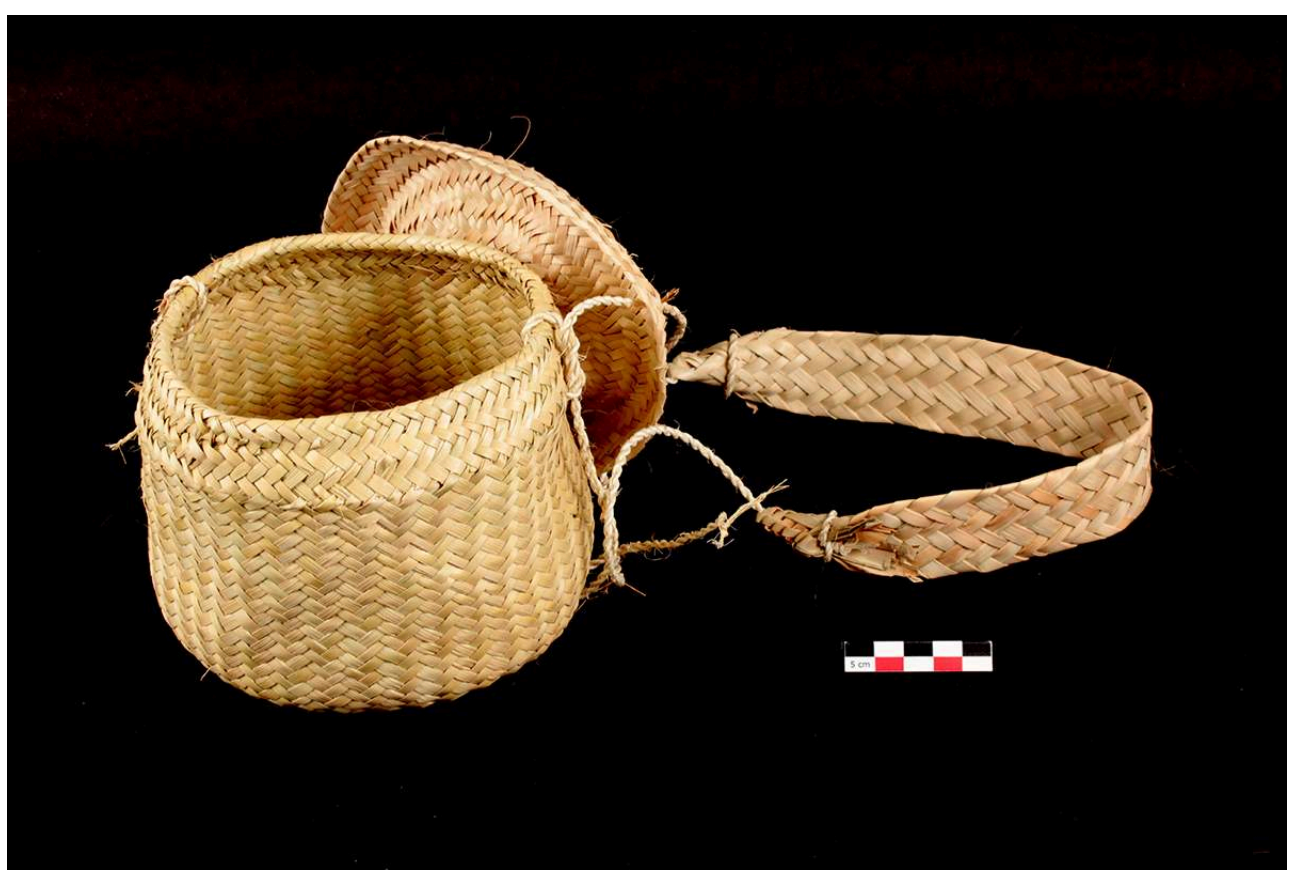

(C) P. Rameau

131 - Zacatlán (Puebla)

- Palmes tressées; se porte au côté pendant les semailles. Vannerie tissée-nattée, en diagonale

- 23/8/2015; collecteur Serge Bahuchet

- $27 \mathrm{H} 30 \mathrm{~cm}$ (sans la sangle)

- ETB-MX-SB-2015-029

132 Ces paniers tenate peuvent également servir de petite hotte, portée par des enfants avec un bandeau frontal ( $c f$. Figure 90).

133 36) Petit panier à semences 


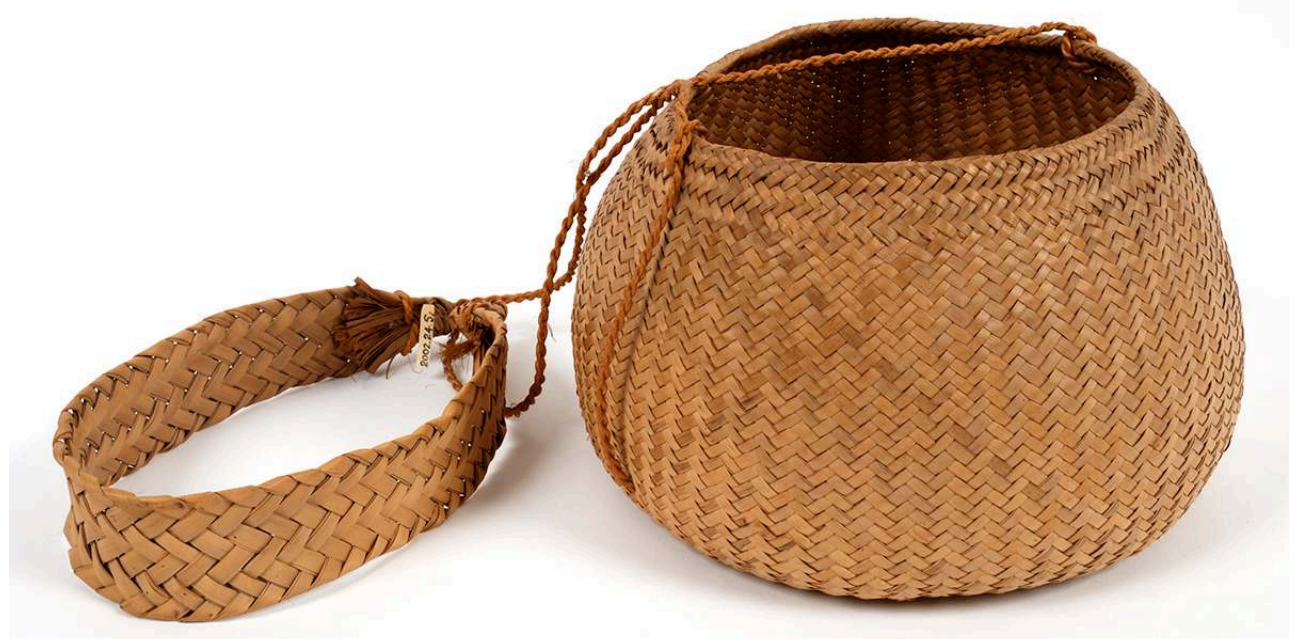

(C) D. Brabant

134 - Nahua. Huauchinango (Puebla)

- Sac à semences tressé en fibres végétales avec bandoulière tressée et cordelettes végétales. Sierra Norte de Puebla.

-1970, collectrice Marie Noëlle Chamoux

- diamètre $22 \mathrm{~cm}$, hauteur $14,5 \mathrm{~cm}$; bandeau: 54 x 4,3 cm

- MNHN-E-2002.24.5 (Exposition permanente Galerie de l'Homme)

37) Petit panier à semences (bolsa palma)

Figure 82 : Petit panier à semences ETB-MX-SB-2016-014

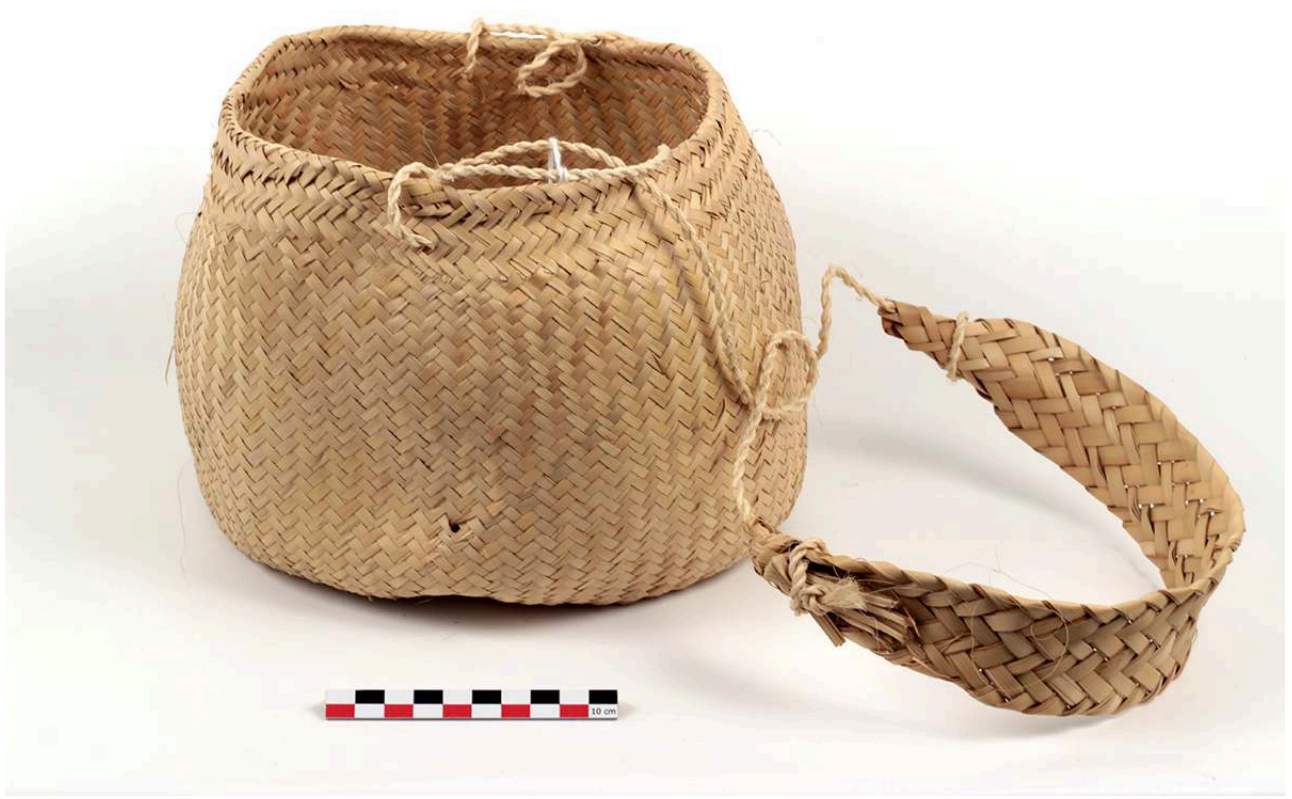

(C) P. Rameau 
- Palme tressée

$\cdot 1 / 2 / 2016$; collecteur Serge Bahuchet

- $~ 21, \mathrm{H} 17 \mathrm{~cm}$ (sans la sangle)

• ETB-MX-SB-2016-014

38) Sac à semences

Figure 83 : Sac à semences ETB-MX-PR-2016-006

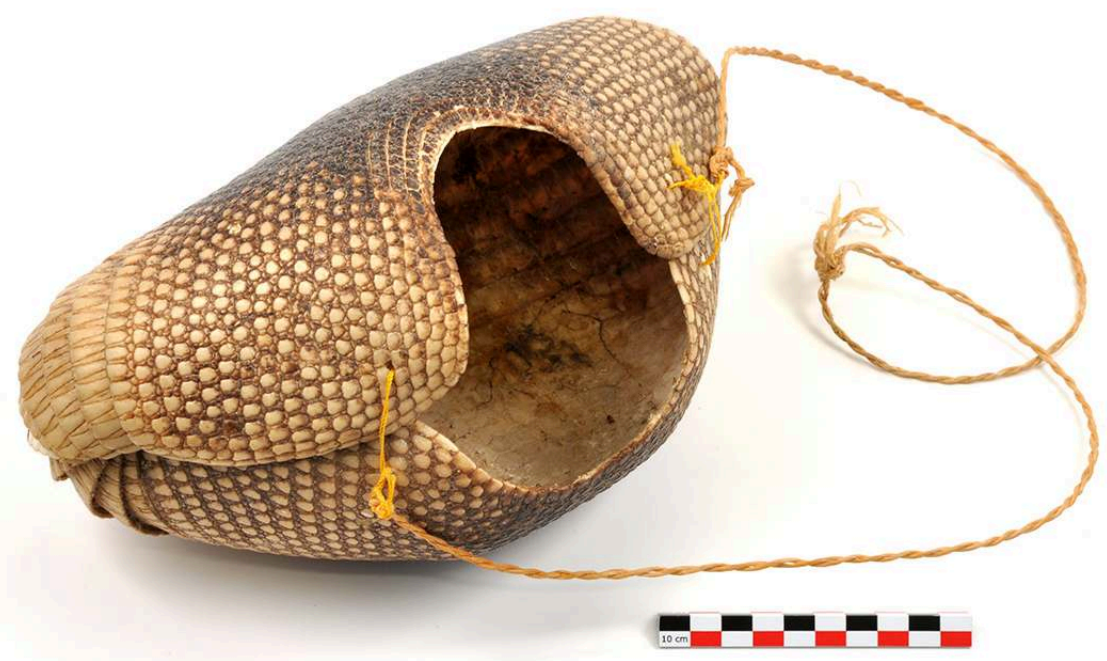

(C) P. Rameau

- Altopesco, la Huasteca (Hidalgo)

- Carapace de tatou (Dasypus novemcinctus, Dasypodidae)

-23/6/2016 ; collectrice Pauline Rameau, don d'Alvaro Salgado

- 27 x 12 × $17 \mathrm{~cm}$

- ETB-MX-PR-2016-006

L'anthropologue Frederick Starr rapporte cette observation des sacs en carapace de tatou et de leur préparation, dans des villages Otomi et «Aztèques " (Nahuatl) du district de Huachinango (Puebla) qu'il parcourt en 1898 :

At many villages and at scattered country houses in this region they have quaint sacks made of armadillo shell. The shell is removed and bent while fresh into the desired shape; it is packed tightly full of ashes to make it retain its shape while drying. It is then hung to a cord which passes over the shoulder. It is used only for holding seed corn at planting time. (Figure 68.) (Starr 1899-1900: 80)

\section{L'entretien du champ}

Au cours de la croissance des jeunes tiges de maïs, le paysan procède régulièrement au désherbage, pour nettoyer le champ. Il emploie pour cela sa main, sa machette, une serpette, ou encore une houe selon les régions (Figure 62), mais aussi araire ou charrue pour les grandes parcelles (Figure 85). Les «mauvaises herbes ", ces rudérales, ainsi 
dégagées ne sont pas pour autant perdues, beaucoup d'espèces constituent des quelites ${ }^{14}$ très appréciées, que l'on va consommer après cuisson (Bye 2000).

Figure 84 : « Milpa nahuatl », désherbage à la main (27/6/2018, Hidalgo)

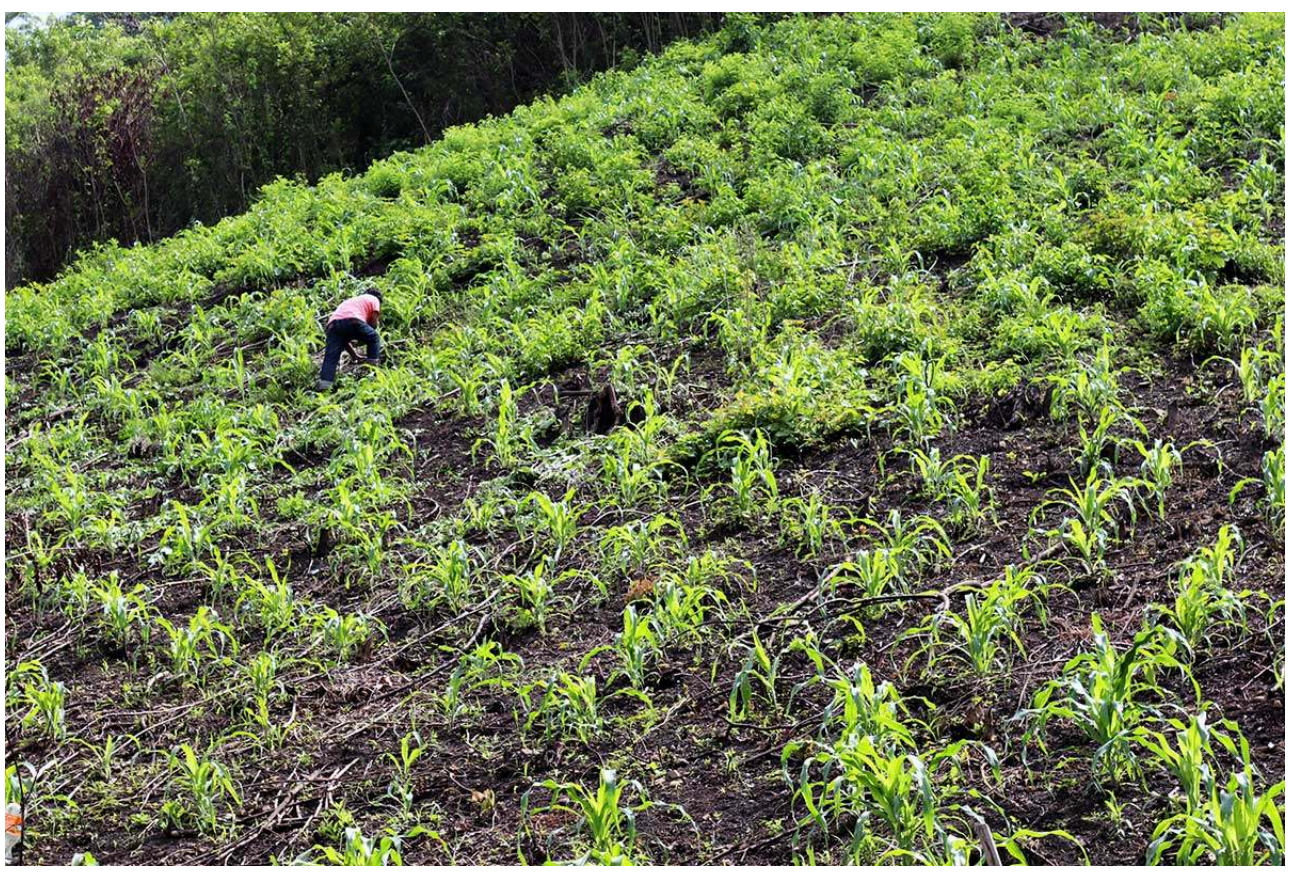

(C) P. Rameau

Figure 85 : « La limpia de la milpa » : nettoyer les maïs des mauvaises herbes, à la main et avec une charrue (milpa de Camilo 2, 11/7/201, Chiquilistlán, Jalisco)

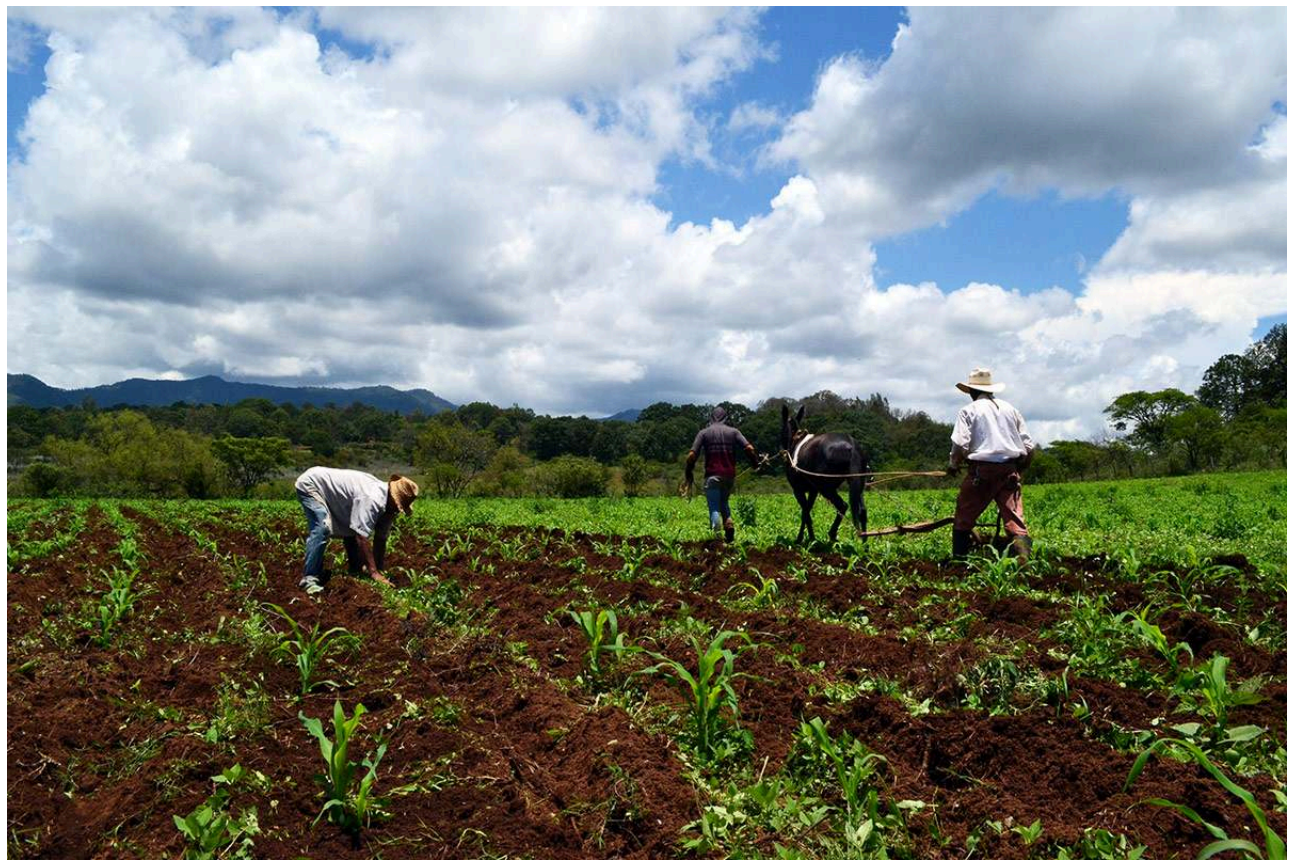

(c) P. Rameau

$141 \quad 39)$ Serpettes 

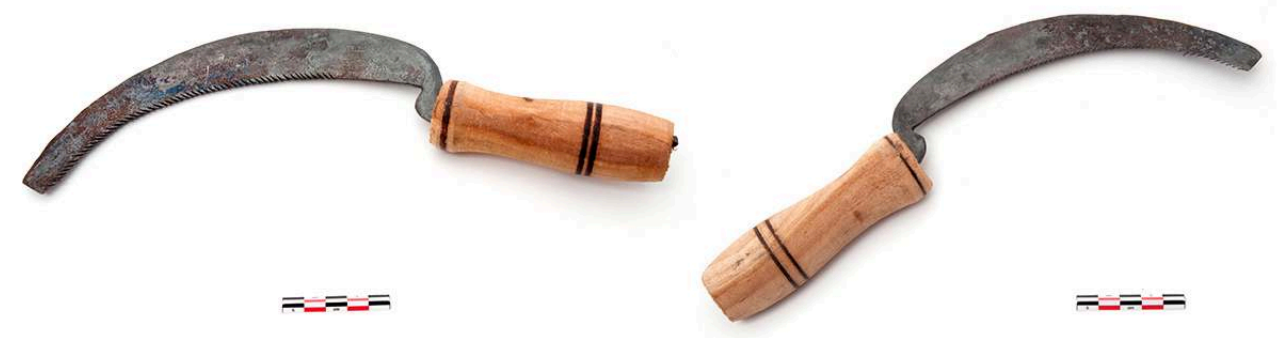

À gauche : ETB-MX-SB-2010A-020, Guadalajara (Jalisco), L 28 cm, 16/4/2010

À droite : ETB-MX-SB-2010B-011, Tequila (Jalisco), L 28 cm, 13/11/2010

Lame dentée en métal, manche en bois

Bien qu'achetées dans des lieux différents, ces deux serpettes proviennent du même atelier ; elles servent autant à désherber le champ qu'à couper les feuilles de maïs pour nourrir les animaux

(c) J.-C. Domenech

\section{Fin de la première partie}

À suivre, $2^{\mathrm{e}}$ section: la récolte, la conservation; liste des objets par régions; remerciements ; annexe « liste des variétés de maïs récoltées »; bibliographie

\section{NOTES}

1. La collection d'objets et spécimens mexicains représente environ $17 \%$ du matériel d'étude d'ethnobiologie ; c'est l'une des plus importantes.

2. La spécialité de master Environnement, développement, territoires, sociétés (EDTS), dispensée au MNHN et à AgroParistech, nécessitait que les étudiants en $2^{\mathrm{e}}$ année consacrent leur dernier semestre à un stage de recherche sur le terrain, pour valider leur diplôme.

3. Dans la section «Cultures culinaires", cette évocation d'une foire alimentaire illustrait la reconnaissance de la cuisine mexicaine du Michoacán dans le Patrimoine immatériel de l'Humanité de l'Unesco.

4. «Coamil (sing.) : (Del azt. cuahuitl, árbol, y milli, heredad.) m. Huerta con arboleda. Usual principalmente en la zonas aztecas del país. » (Santamaria, Dicc. Mexicanismos, 2000).

5. C'est cette association des trois plantes qui constitue l'archétype de la milpa.

6. Deux manteaux de pluie Nahua des anciennes collections du Musée de l'Homme sont désormais conservés au Musée du quai Branly, provenant de la même région: 71.1938.152.4 collecté par Robert Gessain à Puebla en 1938, et 71.1977.106.532, donné par la Mission archéologique française au Mexique, dirigée par Guy Stresser-Péan et collecté par lui à Pahuatlán (Puebla).

7. Se siembra en marzo, arrojando cuatro o cinco granos en hoyos distantes un paso entre sí, y vuelve a sembrarse en noviembre, diciembre y enero, labrando de nuevo la tierra; (...) se cosecha más tarde o más pronto y se guarda desgranado. (Barros \& Buenrostro 2007 : 92) 
8. Instrument agricole qui ressemble à une houe, composé d'un long manche de bois avec un fer terminé en pointe, dont un côté droit et l'autre courbe. (Notre traduction)

9. Instrument agricole qui sert à creuser, comme la pelle. Il consiste en une pièce, plus ou moins triangulaire, comme une pelle plate ou légèrement cannelée, de fer épais et aiguisé à la base ; avec un manche long et lourd, comme une pique, et que l'on manipule en se tenant debout. (Notre traduction)

10. Coa : instrument de travail agricole, comme une houe. Tarécua : Il existe un instrument agricole avec une pointe courbe et affûtée sur les deux côtés, que l'on nomme coa au Yucatan et tarécua au Michoacan, un terme dérivé du tarasque tarhekua, outil avec lequel on creuse ou on laboure pour ensemencer, de tarheni, creuser ou labourer le champ.

11. Actuellement, les coas ne sont plus en usage chez les P'urhépecha, et tarekwa désigne désormais l'araire, d'ailleurs peu à peu remplacé par le tracteur (à Cherán, F. Grisanti \& T. González García, comm. pers. 8/7/21)

12. Le Diccionario de Mexicanismos de Santamaría réfère à une étymologie nahuatl différente: « coahuitl, palo » (s.v. Coa)

13. Deux autres coas à lame asymétrique, collectées par Guy Stresser-Péan, sont conservées au Musée du quai Branly: une lame nue semi-ovale sans pointe (70.2011.21.605, Totonaque, Xicotepec, Puebla) et une semi-circulaire à pointe décentrée, avec un curieux manche arqué long de $87 \mathrm{~cm}$ (70. 2011.21.607, Nahua, Acaxochitlán, Hidalgo).

14. Quelites est le nom générique pour l'ensemble des herbacées rudérales, sauvages ou non, qui sont comestibles.

\section{RÉSUMÉS}

Les collections mexicaines liées au maïs, au Musée de l'Homme, comptent plus de 250 objets, relevant tant du matériel d'étude que des collections patrimoniales. Après une présentation des recherches menées et des conditions et lieux d'obtention de cette collection, nous décrivons ici ces objets, en les resituant dans leur contexte technique, en y associant de nombreuses photographies de terrain. Le catalogue est divisé en deux parties. Les domaines abordés sont la production agricole, la préparation alimentaire, la consommation, et les aspects liés à la représentation.

Les objets liés au maïs, dans leur diversité, sont présents dans tous les domaines de la culture matérielle. Ainsi, à travers ce catalogue, c'est une certaine évocation de la vie quotidienne du Mexique contemporain que nous esquissons.

Las colecciones mexicanas vinculadas al maíz, en el Museo del Hombre, cuentan con más de 250 objetos, constituyendo éste tanto material de estudio como elemento parte de colecciones patrimoniales. Después de una presentación de las investigaciones desarrolladas y de las condiciones y los lugares de obtención de esta colección, describimos los objetos, situándolos en su contexto técnico, y asociándolos a numerosas fotografías de campo. El catálogo se divide en dos partes principales. Los temas abordados son la producción agrícola, la preparación alimentaria, la consumación y los aspectos relacionados a la representación.

Los objetos vinculados al maíz, en su diversidad, están presentes en todos los sectores de la cultura material. Así, a través de este catálogo, ofrecemos un bosquejo de la vida cotidiana del México contemporáneo. 


\section{AUTEURS}

\section{PAULINE RAMEAU}

Chercheure associée et assistante de gestion des collections d'ethnobiologie, laboratoire Ecoanthropologie (MNHN)

\section{SERGE BAHUCHET}

Professeur d'ethnobiologie du Muséum, laboratoire Eco-anthropologie (MNHN) 